\title{
Weak mixing implies weak mixing of higher orders along tempered functions
}

\author{
Vitaly Bergelson* \\ Department of Mathematics \\ The Ohio State University \\ Columbus, OH 43210, USA \\ vitaly@math.ohio-state.edu
}

\author{
Inger J. Håland Knutson \\ Department of Mathematical Sciences \\ University of Agder \\ N-4604 Kristiansand, Norway \\ inger.j.knutson@uia.no
}

September 28, 2008

\begin{abstract}
We extend the weakly mixing PET (polynomial ergodic theorem) obtained in [2] to much wider families of functions. Besides throwing new light on the question of "how much higher degree mixing is hidden in weak mixing", the obtained results also show the way to possible new extensions of the polynomial Szemerédi theorem obtained in [6].
\end{abstract}

\section{Introduction}

A measure-preserving system $(X, \mathcal{B}, \mu, T)$ (in this paper we will always assume that $(X, \mathcal{B}, \mu)$ is a probability space) is called weakly mixing if for any $A, B \in \mathcal{B}$ one has

$$
\lim _{N \rightarrow \infty} \frac{1}{N} \sum_{n=1}^{N}\left|\mu\left(A \cap T^{-n} B\right)-\mu(A) \mu(B)\right|=0 .
$$

The notion of weak mixing has many equivalent forms and plays an important role in the study and applications of dynamical systems. In particular, weak mixing, and more generally, relative weak mixing play crucial role in the analysis of general measure-preserving systems which is behind Furstenberg's ergodic approach to Szemerédi's theorem (see [13]). In particular, Furstenberg proves in [13] the following theorem.

Theorem 1.1 If an invertible measure preserving system $(X, \mathcal{B}, \mu, T)$ is weakly mixing then for any $k \in \mathbf{N}$ and for any $A_{0}, \ldots, A_{k} \in \mathcal{B}$ one has

$$
\lim _{N \rightarrow \infty} \frac{1}{N} \sum_{n=1}^{N} \mu\left(A_{0} \cap T^{n} A_{1} \cap \cdots \cap T^{k n} A_{k}\right)=\mu\left(A_{0}\right) \mu\left(A_{1}\right) \cdots \mu\left(A_{k}\right) .
$$

${ }^{*}$ Supported by NSF grants DMS-0345350 and DMS-0600042 
Theorem 1.1 can be given the following equivalent form. (In accordance with the well established tradition, we write $T f$ for $f(T x)$.)

Theorem 1.2 If $(X, \mathcal{B}, \mu, T)$ is an invertible weakly mixing system, then for any $k \in \mathbf{N}$ and any $f_{1}, f_{2}, \ldots, f_{k} \in L^{\infty}(X, \mathcal{B}, \mu)$

$$
\lim _{N \rightarrow \infty}\left\|\frac{1}{N} \sum_{n=1}^{N} T^{n} f_{1} T^{2 n} f_{2} \cdots T^{k n} f_{k}-\prod_{i=1}^{k} \int f_{i} d \mu\right\|_{2}=0 .
$$

The following extension of Theorem 1.2 which was proved in [2] shows that weak mixing implies weak mixing of higher orders along polynomials.

Theorem 1.3 Suppose that $(X, \mathcal{B}, \mu, T)$ is an invertible weakly mixing system and let $p_{1}, \ldots, p_{k}$ be pairwise essentially distinct polynomials (i.e. $p_{i}-p_{j} \not \equiv$ constant for all $i \neq j$ ) with rational coefficients and taking on integer values on the integers. Then for any $f_{1}, \ldots, f_{k} \in L^{\infty}(X, \mathcal{B}, \mu)$ one has:

$$
\lim _{N \rightarrow \infty}\left\|\frac{1}{N} \sum_{n=1}^{N} T^{p_{1}(n)} f_{1} T^{p_{2}(n)} f_{2} \cdots T^{p_{k}(n)} f_{k}-\prod_{i=1}^{k} \int f_{i} d \mu\right\|_{2}=0 .
$$

The goal of this paper is extending Theorem 1.3 to classes of sequences which are more general than those of the form $p(n)$, where $p$ is a polynomial satisfying $p(\mathbf{Z}) \subset \mathbf{Z}$. While there are relatively few candidates among sufficiently regular functions which have the property that they take integer values at integers ${ }^{1}$, one may try to introduce into the picture sequences of the form $[g(n)]$, where $g$ is a continuous eventually monotone function which is not too slow and not too fast ${ }^{2}$, and satisfies some natural regularity conditions. As we will show, functions which "fit the profile" can be found in the family of the so called tempered functions which we will presently define. Before starting with technicalities (which are needed in order to give precise formulations) we want to note that our typical theorems will look very much like (2) with $p_{i}(n)$ replaced by $\left[g_{i}(n)\right]$, where the $g_{i}$ will be coming from quite wide families of functions which include the integer-valued polynomials as rather small subfamily. The obtained results not only throw new light on the old question of "how much higher degree mixing is hidden in weak mixing", but also will, hopefully, be useful for further extensions of Szemerédi's theorem.

Definition 1.4 (Cf. [11]) Let $i$ be a non-negative integer. A real valued function $g$ which is $(i+1)$ times continuously differentiable on $\left[x_{0}, \infty\right)$, where $x_{0} \geq 0$, is called a tempered function of order $i$ if

(a) $g^{(i+1)}(x)$ tends monotonically to zero as $x \rightarrow \infty$, and

(b) $\lim _{x \rightarrow \infty} x\left|g^{(i+1)}(x)\right|=\infty$.

Tempered functions of order 0 are called Fejér functions.

\footnotetext{
${ }^{1}$ One can show, for example, that if $f$ is an entire function such that $f(\mathbf{Z}) \subset \mathbf{Z}$, then it is either a polynomial or has exponential growth. (See the next footnote with regards to the exponential growth.)

${ }^{2}$ We have to eliminate too slow (say, like $\log n$ ) and too fast (say, $a^{n}$, where $a>1$ ) functions since, for these functions, the Cesàro averages $\frac{1}{N} \sum_{n=1}^{N} T^{[g(n)]} f$ may fail to converge.
} 
We note that the conditions (a) and (b) imply that tempered functions are eventually monotone, and that positive tempered functions of order $i$ increase at least as fast as $x^{i} \log x,{ }^{3}$ but they are slower than $x^{i+1}$.

The following theorem explains the role of Fejér functions in the theory of uniform distribution (see [19, Cor. 2.1]).

Theorem 1.5 (Fejér) Let $g(x)$ be a differentiable function for $x \geq x_{0}$, where $x_{0} \geq 0$. If $g^{\prime}(x)$ tends monotonically to 0 as $x \rightarrow \infty$ and if $\lim _{x \rightarrow \infty} x\left|g^{\prime}(x)\right|=\infty$, then the sequence $g(n)$, $n=1,2, \ldots$, is uniformly distributed modulo 1 .

Remark: By using van der Corput's difference theorem (which states that if the sequence $x_{n+h}-x_{n}, n=1,2, \ldots$, is uniformly distributed $(\bmod 1)$ for any $h \in \mathbf{N}$, then $x_{n}, n=1,2, \ldots$, is uniformly distributed $(\bmod 1))$, together with Fejér's theorem, one can easily see that $g(n)$, $n=1,2, \ldots$, is uniformly distributed $(\bmod 1)$ if $g$ is a tempered function. (See $[21, \text { p. 381-3] })^{4}$.

The following classes of functions will be instrumental for our work. Note that the first class is a subset of the set of Fejér functions.

\section{Definition 1.6}

$$
\begin{aligned}
\mathcal{F} & =\left\{g \in C^{\infty}\left(\mathbf{R}^{+}\right) \mid g \text { is Fejér and } \exists \alpha \in(0,1] \text { such that } \lim _{x \rightarrow \infty} \frac{x g^{\prime \prime}(x)}{g^{\prime}(x)}=\alpha-1\right\} \\
\mathcal{L} & =\left\{g \in C^{\infty}\left(\mathbf{R}^{+}\right) \mid \exists \gamma \neq 0 \text { such that } \lim _{x \rightarrow \infty} g^{\prime}(x)=\gamma\right\}
\end{aligned}
$$

Remark: If $g \in \mathcal{F}$ then it follows by l'Hopital's rule that $\lim _{x \rightarrow \infty} \frac{x g^{\prime}(x)}{g(x)}=\alpha$. Also, if $g \in C^{\infty}\left(\mathbf{R}^{+}\right)$and $0<\alpha<1$ such that $\lim _{x \rightarrow \infty} \frac{x g^{\prime \prime}(x)}{g^{\prime}(x)}=\alpha-1$ then $g$ is a Fejér function, see Lemma 2.2 below. However, too slow Fejér functions are not members of $\mathcal{F}$, see examples below. The class $\mathcal{L}$ consists of all functions $g$ such that $g(x) \rightarrow \infty$ and $\lim _{x \rightarrow \infty} \frac{g(x)}{x} \in \mathbf{R} \backslash\{0\}$, see examples below.

We remark that all the theorems in this paper remain true if we only demand that all the functions involved have sufficiently many derivatives.

\section{Examples:}

1. The following function is Fejér but is not in the class $\mathcal{F}$ :

$$
\text { - } \left.g_{1}(x)=\log ^{\beta} x, \beta>1 \text {. (Indeed, } \lim _{x \rightarrow \infty} \frac{x g_{1}^{\prime}(x)}{g_{1}(x)}=0\right) \text {. }
$$

2. The following are functions from $\mathcal{F}$ :

\footnotetext{
${ }^{3}$ Indeed, if $g$ is an increasing Fejér function and $a>0$, then there exists $M$ such that for $t>M, t g^{\prime}(t)>a$, which implies that $g(x)-g(M)=\int_{M}^{x} g^{\prime}(t) d t>\int_{M}^{x} \frac{a}{t} d t=a \log \frac{x}{M}$. Moreover, it follows by induction on $i$ that if $g$ is a tempered function of order $i$, then for given $c>0$, there exists $M$ so that for $x>M, \frac{|g(x)|}{x^{i} \log x}>c$.

${ }^{4}$ This result implies, via the spectral theorem, that if $(X, \mathcal{B}, \mu, T)$ is an ergodic system, and $g$ is a tempered function, then for all $f \in L^{2}(X, \mathcal{B}, \mu)$ one has $\lim _{N \rightarrow \infty}\left\|\frac{1}{N} \sum_{n=1}^{N} T^{[g(n)]} f-\int f\right\|_{2}=0$. See Theorem 7.1 below.
} 
- $g_{2}(x)=\sum_{i=1}^{k} c_{i} x^{\alpha_{i}}, c_{i} \in \mathbf{R}, c_{k} \neq 0, \alpha_{i} \in \mathbf{R}, \alpha_{1}<\alpha_{2}<\cdots<\alpha_{k}, 0<\alpha_{k}<1$.

- $g_{3}(x)=x^{\alpha} \log ^{\beta} x, 0<\alpha<1, \beta \in \mathbf{R}$.

- $g_{4}(x)=\frac{x}{\log ^{\beta} x}, \beta>0$.

- $g_{5}(x)=x^{\alpha}\left(\cos \left(\log ^{\beta} x\right)+2\right), 0<\alpha<1, \beta<1$.

- $g_{6}(x)=x^{\alpha}\left(1+\frac{\sin (\log x)}{\log x}\right), 0<\alpha<1$.

- $g_{7}(x)=x^{\alpha}\left(1+\frac{\cos x}{x^{l}}\right), 0<\alpha<1, l \geq 3$.

3. The following are functions from $\mathcal{L}$ :

- $g_{8}(x)=\sum_{i=1}^{k} c_{i} x^{\alpha_{i}}, c_{i} \in \mathbf{R}, c_{k} \neq 0, \alpha_{i} \in \mathbf{R}, \alpha_{1}<\alpha_{2}<\cdots<\alpha_{k}=1$.

- $g_{9}(x)=x\left(1+\frac{\sin (\log x)}{\log x}\right)$.

- $g_{10}(x)=x\left(1+\frac{\cos x}{x^{l}}\right), l>1$.

As we will see (cf. Theorem 4.3), the analogue of Theorem 1.3 holds true for functions from $\mathcal{F} \cup \mathcal{L}$. An inductive scheme which is similar to that utilized in [2] allows one to extend Theorem 4.3 to wider classes of functions which have the property that after taking enough derivatives they "fall" into the class $\mathcal{F} \cup \mathcal{L}$. This roughly describes one of our major results, Theorem A below, for which the family of functions is a subset of the Hardy field of logarithmicoexponential functions (see Definition 1.7 below). Our other main result, Theorem B, while similar in nature, differs from Theorem A in that the family of functions involved include functions with "oscillation", namely functions of the form $n^{\alpha} f(n)$, where $f$ can have infinitely many changes of the sign of the derivative. The functions themselves are eventually monotone but the ratio of two functions from this family does not even need to converge. This widening of the class of functions comes at the price of minor narrowing which functions can be used together in the theorem. For our proofs to go through, this set of functions need to have some regular behavior.

We will proceed now to give precise formulations.

Definition 1.7 We will use the following classes of functions.

$\mathcal{H}=$ the Hardy field of logarithmico-exponential functions, i.e., real-valued functions defined for all $x>a$ for some $a \in \mathbf{R}$, by a finite combination of symbols ,,$+- \times,:, \sqrt[n]{ }, \log$, exp, acting on the real variable $x$ and on real constants, see $([15],[10])$.

$\mathcal{R}=\left\{g \in C^{\infty}\left(\mathbf{R}^{+}\right) \mid \lim _{x \rightarrow \infty} \frac{x g^{(j+1)}(x)}{g^{(j)}(x)}\right.$ exists and is finite for all $\left.j \geq 0\right\}$

$\mathcal{T}_{i}=\left\{g \in \mathcal{R} \mid \exists i<\alpha \leq i+1, \lim _{x \rightarrow \infty} \frac{x g^{\prime}(x)}{g(x)}=\alpha, \lim _{x \rightarrow \infty} g^{(i+1)}(x)=0\right\}, i=0,1,2, \ldots$

$\mathcal{P}_{i}=\left\{g \in C^{\infty}\left(\mathbf{R}^{+}\right) \mid \exists \gamma \in \mathbf{R} \backslash\{0\}, \lim _{x \rightarrow \infty} g^{(i+1)}(x)=\gamma, \lim _{x \rightarrow \infty} x^{j} g^{(i+j+1)}(x)=0, j \in \mathbf{N}\right\}$, $i=0,1,2, \ldots$

$\mathcal{P}=\bigcup_{i \geq 0} \mathcal{P}_{i}, \quad \mathcal{T}=\bigcup_{i \geq 0} \mathcal{T}_{i}, \quad \mathcal{G}=\mathcal{T} \cup \mathcal{P}$ 


\section{Remarks:}

(i) The class $\mathcal{R}$ is only used to define the classes $\mathcal{T}_{i}$ and will not be used by itself. We remark that all the theorems involving functions from $\mathcal{T}$ remain true if we only demand that sufficiently many of the ratios $\frac{x g^{(j+1)}(x)}{g^{(j)}(x)}$ converge.

(ii) Here is a summary of some properties of the class $\mathcal{T}$ :

(1) All functions in $\mathcal{T}_{i}$ are tempered of order $i, i=0,1,2, \ldots$, see Proposition 5.2 below. Also, $\mathcal{T}_{0} \subsetneq \mathcal{F}$. For example, $x^{\alpha}\left(1+\frac{\cos x}{x^{l}}\right) \in \mathcal{F} \backslash \mathcal{T}_{0}$ when $0<\alpha<1, l \geq 3$.

(2) If $g \in \mathcal{T}_{i}$ then there exist $i<\alpha \leq i+1$ and $f \in C^{\infty}\left(\mathbf{R}^{+}\right)$such that $\lim _{x \rightarrow \infty} \frac{x^{j} f^{(j)}(x)}{f(x)}=0$ for all $j \in \mathbf{N}$ and $g=x^{\alpha} f$, see Proposition 5.4 below.

(3) $g \in \mathcal{T}_{i} \Leftrightarrow x g \in \mathcal{T}_{i+1}, i=0,1, \ldots$

(iii) Here is a summary of properties of the class $\mathcal{P}$ :

(1) The set $\mathcal{P}_{0}$ consists of all the functions $g \in \mathcal{L}$ which satisfy the regularity condition that $\lim _{x \rightarrow \infty} x^{j} g^{(i+j+1)}(x)=0, j \in \mathbf{N}$. Since, for example, $x\left(1+\frac{\cos x}{x^{l}}\right) \in \mathcal{L} \backslash \mathcal{P}_{0}$ when $l \geq 2, \mathcal{P}_{0} \subsetneq \mathcal{L}$. The set $\mathcal{P}_{i}$ contains, among other functions, all the polynomials of degree $i+1$. Note that $\mathcal{P}$ does not contain constants.

(2) If $g \in \mathcal{P}_{i}$ then there exist $\gamma \in \mathbf{R} \backslash\{0\}$ and $f \in C^{\infty}\left(\mathbf{R}^{+}\right)$such that $\lim _{x \rightarrow \infty} f(x)=\gamma$, $\lim _{x \rightarrow \infty} x^{j} f^{(j)}(x)=0$ for all $j \in \mathbf{N}$ and $g=x^{i+1} f$, see Proposition 5.4 below.

(3) $g \in \mathcal{P}_{i} \Leftrightarrow x g \in \mathcal{P}_{i+1}, i=0,1, \ldots$

(iv) To get examples of functions from $\mathcal{T}_{i}$ and $\mathcal{P}_{i}$ one can just multiply the functions $g_{2}, \ldots, g_{6}$, $g_{8}, g_{9}$ in the examples following Definition 1.6 above by $x^{i}$.

(v) Let $\alpha>0$ and $\beta \in \mathbf{R}$, and let $g(x)=x^{\alpha} \log ^{\beta} x$. Then $g \in \mathcal{H}$. If $\alpha \notin \mathbf{N}$ then $g \in \mathcal{T}_{[\alpha]}$, and hence, $g \in \mathcal{T}$. However, if $\alpha=l \in \mathbf{N}$, then $g$ is a tempered function only for $\beta>1$ and $\beta<0$. The following table summarizes the data about the dependence of $g$ on the parameter $\beta$ when $\alpha \in \mathbf{N}$ :

$$
\begin{array}{rlrl}
\beta \neq 0: & & g \in \mathcal{R} \\
\beta>1: & & g \text { is tempered of order } l \text { but is not in the class } \mathcal{T} \\
0<\beta \leq 1: & & g \text { is not tempered and is not in the class } \mathcal{G} \\
\beta & =0: & & g \text { is a polynomial } \\
\beta<0: & & g \in \mathcal{T}_{l-1}
\end{array}
$$

Hardy's logarithmico-exponential functions have the property that they are eventually monotone. This fact is conveniently utilized in the proofs of theorems which involve $\mathcal{H}$ and also leads to more streamlined formulations. Here is the formulation of one of the extensions of Theorem 1.3 to be proved in this paper. 
Theorem A Let $g_{1}, \ldots, g_{k} \in \mathcal{G} \cap \mathcal{H}$ be such that $g_{i}-g_{j} \in \mathcal{G}$ for $i \neq j$. Then for any invertible weakly mixing system $(X, \mathcal{B}, \mu, T)$ and any $f_{1}, \ldots, f_{k} \in L^{\infty}(X, \mathcal{B}, \mu)$,

$$
\lim _{N \rightarrow \infty}\left\|\frac{1}{N} \sum_{n=1}^{N} T^{\left[g_{1}(n)\right]} f_{1} \cdots T^{\left[g_{k}(n)\right]} f_{k}-\prod_{i=1}^{k} \int f_{i}\right\|_{2}=0 .
$$

Corollary 1.8 Let $_{g_{1}}, \ldots, g_{k} \in \mathcal{G} \cap \mathcal{H}$ be such that $g_{i}-g_{j} \in \mathcal{G}$ for $i \neq j$. Then for any invertible weakly mixing system $(X, \mathcal{B}, \mu, T)$, any $\varepsilon>0$ and any $A \in \mathcal{B}$,

$$
\left\{n \in \mathbf{N}|| \mu\left(A \cap T^{\left[g_{1}(n)\right]} A \cap \cdots \cap T^{\left[g_{k}(n)\right]} A\right)-(\mu(A))^{k+1} \mid<\varepsilon\right\}
$$

has density $1 .^{5}$

\section{Remarks:}

1. We have $\mathcal{G} \cap \mathcal{H}=\left\{g \in \mathcal{H} \mid \exists \alpha>0, \lim _{x \rightarrow \infty} \frac{x g^{\prime}(x)}{g(x)}=\alpha\right.$, if $\alpha \in \mathbf{N}$ then $\left.\lim _{x \rightarrow \infty} g^{(\alpha)}(x) \in \mathbf{R}\right\}$, see Proposition 5.1 below. Note that $\mathcal{G} \not \subset \mathcal{H}$. For example, if $\alpha>0, \alpha \notin \mathbf{N}$, then $g(x)=x^{\alpha}(2+\cos \sqrt{\log x}) \in \mathcal{G} \backslash \mathcal{H}$. Note also that $g$ cannot be a member of a Hardy field since the derivative of $g^{2}$ is not eventually monotone.

2. Hardy fields also appear in [9] where the authors study the generalizations of the classical von Neumann and Birkhoff ergodic theorems. While there is practically no overlap between our paper and [9], the two papers demonstrate, each in its own way, that Hardy fields provide a natural framework for extensions of some of the familiar ergodic results.

3. For a measure preserving system with continuous time, Theorem A, as well as Theorem B below and many other theorems in this paper, admits two additional versions dealing with the discrete averages of the form $\frac{1}{N} \sum_{n=1}^{N} T^{g_{1}(n)} f_{1} T^{g_{2}(n)} f_{2} \cdots T^{g_{k}(n)} f_{k}$ or continuous averages of the form $\frac{1}{T} \int_{0}^{T} T^{g_{1}(t)} f_{1} \cdots T^{g_{k}(t)} f_{k} d t$. All three versions have essentially the same proofs. A similar phenomenon was observed (with regards to mean convergence) in the paper [9] alluded to above.

In order to formulate our second main theorem, we need a further refinement of our classes.

Definition 1.9 Let $\alpha>0$. Then

$$
\begin{aligned}
& \mathcal{T}(\alpha)=\left\{g \in \mathcal{T} \mid \lim _{x \rightarrow \infty} \frac{x g^{\prime}(x)}{g(x)}=\alpha\right\} \\
& \mathcal{G}(\alpha)=\left\{g \in \mathcal{G} \mid \lim _{x \rightarrow \infty} \frac{x g^{\prime}(x)}{g(x)}=\alpha\right\}
\end{aligned}
$$

Note that $\mathcal{T}(l) \subset \mathcal{T}_{l-1}$ and that $\mathcal{G}(l)=\mathcal{T}(l) \cup \mathcal{P}_{l-1}$ when $l \in \mathbf{N}$.

To formulate our next definition we need the following notation.

\footnotetext{
${ }^{5}$ The density of a subset $E \subset \mathbf{N}$ is defined by $d(E)=\lim _{N \rightarrow \infty} \frac{|E \cap[1, N]|}{N}$ when this limit exists. Below we will also use the the upper density of a subset $E \subset \mathbf{N}$ defined by $\bar{d}(E)=\limsup _{N \rightarrow \infty} \frac{|E \cap\{1, \ldots, N\}|}{N}$ and the lower density $\underline{d}(E)$ which is defined similarly but with lim inf instead of lim sup.
} 
- $G_{\text {diff }}=\left\{g_{1}-g_{2} \mid g_{1}, g_{2} \in G, g_{1} \neq g_{2}\right\}$ when $G$ is a finite set of functions.

- If $\alpha>0$ and $G \subset \mathcal{G}$, then $G(\alpha)=G \cap \mathcal{G}(\alpha)$.

Definition 1.10 A finite set $G \subset \mathcal{G}$ for which $G_{\mathrm{diff}} \subset \mathcal{G}$ has the R-property ( $R$ for regularity) if for all $\alpha>0$ and all pairs $\psi_{1}, \psi_{2} \in G(\alpha) \cup G(\alpha)_{\text {diff }}$ such that if $l \in \mathbf{N} \cup\{0\}$ and $\beta \leq \alpha$ with $\psi_{1}^{(l)}, \psi_{2} \in \mathcal{T}(\beta)$, then

$$
\lim _{x \rightarrow \infty} \frac{\psi_{1}^{([\beta]+l+1)}(x)}{\psi_{2}^{([\beta]+1)}(x)} \in \mathbf{R} \backslash\{0\} .
$$

\section{Remarks:}

- The R-property regulates the relations between those functions in $G$ which have similar rates, since if $\lim _{x \rightarrow \infty} \frac{x g^{\prime}(x)}{g(x)}=\alpha$ then $g(x)=x^{\alpha} f(x)$, where $f$ is either bounded or grows slower than any function in $\mathcal{T}_{0}$. (See Proposition 5.4).

- By Lemma 5.8 below, if $\psi_{1}^{(l)}, \psi_{2} \in \mathcal{T}(\beta)$ satisfy (3) above, then $\lim _{x \rightarrow \infty} \frac{\psi_{1}^{(l+i)}(x)}{\psi_{2}^{(i)}(x)} \in \mathbf{R} \backslash\{0\}$ for all $i \geq 0$. If $\beta \notin \mathbf{N}$ then (3) may be replaced by the equivalent condition that $\lim _{x \rightarrow \infty} \frac{\psi_{1}^{(l)}(x)}{\psi_{2}(x)} \in \mathbf{R} \backslash\{0\}$.

Theorem B Let $g_{1}, \ldots, g_{k} \in \mathcal{G}$ be such that $g_{i}-g_{j} \in \mathcal{G}$ for $i \neq j$ and such that the family $\left\{g_{1}, \ldots, g_{k}\right\}$ has the $R$-property. Then for any invertible weakly mixing system $(X, \mathcal{B}, \mu, T)$ and any $f_{1}, \ldots, f_{k} \in L^{\infty}(X, \mathcal{B}, \mu)$,

$$
\lim _{N \rightarrow \infty}\left\|\frac{1}{N} \sum_{n=1}^{N} T^{\left[g_{1}(n)\right]} f_{1} \cdots T^{\left[g_{k}(n)\right]} f_{k}-\prod_{i=1}^{k} \int f_{i}\right\|_{2}=0 .
$$

\section{Examples:}

The following sets of functions, which are subsets of $\mathcal{G}$ but not of $\mathcal{H}$, have the R-property and hence satisfy Theorem B:

- $\left\{x^{\alpha_{1}}, 2 x^{\alpha_{1}}, x^{\alpha_{1}}\left(3+\frac{\sin (\log x)}{\log x}\right), x^{\alpha_{2}}(2+\cos \sqrt{\log x})\right\} \subset \mathcal{G}$, where $0<\alpha_{1}<\alpha_{2}, \alpha_{2} \notin \mathbf{N}$.

- $\left\{\sqrt[3]{x}\left(1+\frac{1}{\log x}\right), \sqrt[5]{x}\left(3+\frac{\sin (\log x)}{\log x}\right), \sqrt{x^{3}}-x, \sqrt[4]{x^{3}}(2+\cos \sqrt{\log x})\right\} \subset \mathcal{T}_{0} \cup \mathcal{T}_{1}$

- $\left\{\frac{x}{\log x}, x, x\left(2+\frac{\sin (\log x)}{\sqrt{x}}\right)\right\} \subset \mathcal{T}_{0} \cup \mathcal{P}_{0}$

Corollary 1.11 Let $0<\alpha_{1}<\cdots<\alpha_{l}$ and let $\psi_{i} \in \mathcal{G}\left(\alpha_{i}\right), i=1, \ldots, l$.

- Then $\left\{\psi_{1}, \ldots, \psi_{l}\right\}$ satisfies Theorem $B$.

- Suppose that $\alpha_{i}-\alpha_{j} \notin \mathbf{Z}$ for all $i \neq j$, and let $\Psi=\left\{\sum_{i=1}^{l} a_{i} \psi_{i} \mid a_{i} \in \mathbf{R}, i=1, \ldots, l\right\} \backslash\{0\}$. If $g_{1}, \ldots, g_{k} \in \Psi$ such that $g_{i}-g_{j} \in \Psi$ for all $i \neq j$, then $\left\{g_{1}, \ldots, g_{k}\right\}$ satisfies Theorem $B$. 


\section{Examples:}

The following sets of functions do not have the R-property and we do not know if Theorem B holds true for these sets:

(1) $\{\sqrt{x}, \sqrt{x}(3+\cos \sqrt{\log x})\} \subset \mathcal{T}\left(\frac{1}{2}\right) \subset \mathcal{T}_{0}$ (the ratio of these two functions does not have a limit).

(2) $\left\{x^{3 / 2}, x^{3 / 2}-x^{1 / 2}(3+\cos \sqrt{\log x})\right\} \subset \mathcal{T}\left(\frac{3}{2}\right) \subset \mathcal{T}_{1}$ (the ratio of the derivative of the first function and the difference of the functions does not have a limit).

(3) $\left\{g_{1}(x)=\sqrt{x} \log x, g_{2}(x)=\sqrt{x}(2+\cos \sqrt{\log x})\right\} \subset \mathcal{T}\left(\frac{1}{2}\right) \subset \mathcal{T}_{0}$ (the ratio $g_{2}(x) / g_{1}(x)$ goes to 0$)$.

The following brief comment is intended to explain the reader why our methods are not sufficient for verifying whether the functions in the above examples are "good" for Theorem B. We will focus on Example (1); analysis of the other two examples reveals similar troubles with the proof. Consider the expression

$$
\frac{1}{N} \sum_{n=1}^{N} T^{[\sqrt{n}]} f_{1} T^{[\sqrt{n}(3+\cos \sqrt{\log n})]} f_{2} .
$$

Since both $[\sqrt{n}]$ and $[\sqrt{n}(3+\cos \sqrt{\log n})]$ are of sublinear growth, the only available to us method of starting the verification of the convergence of (4) is to perform first the change of variables $m=[\sqrt{n}]$ which leads to a new expression containing a linear function. This change of variables in (4) gives the new expression

$$
\frac{1}{N} \sum_{n=1}^{N} T^{n} f_{1} T^{\left[n\left(3+\cos \sqrt{\log n^{2}}\right)\right]} f_{2} .
$$

But, unfortunately, the function $x\left(3+\cos \sqrt{\log x^{2}}\right)$ does not belong to $\mathcal{G}$ and it is not clear how to proceed further.

Note that class $\mathcal{T}$ does not contain the "slow" Fejér functions, such as, say, $\log ^{2} x$ (nor does it contain functions which after finitely many differentiations reduce to the "slow" Fejér functions). It would be certainly of interest to extend Theorem B to a more general than $\mathcal{T}$ class of functions and get rid of (or relax) condition R. This, however, would require introduction of new methods and ideas. ${ }^{6}$ Nevertheless, we do obtain in Section 5 the following result which deals with general tempered functions.

Corollary 5.14 Let $g_{1}, \ldots, g_{k}$ be tempered functions and assume that they satisfy one of the following four conditions.

(a) $g_{i}=a_{i} g, i=1, \ldots, k$, where $g$ is a tempered function and $a_{1}<a_{2}<\cdots<a_{k}$.

(b) $g_{i}(x)=g\left(x+a_{i}\right), i=1, \ldots, k$, where $g$ is tempered of order at least one and $0 \leq a_{1}<$ $a_{2}<\cdots<a_{k}$.

\footnotetext{
${ }^{6}$ See Conjecture 8.1 below
} 
(c) $g_{i}$ is tempered of order $i, i=1, \ldots, k$.

(d) If $l \in \mathbf{N}$ and $\phi$ is tempered of order $l$, let $\Omega=\left\{\sum_{i=0}^{l} a_{i} \phi^{(i)} \mid a_{i} \in \mathbf{R}\right\} \backslash\{0\}$, and let $g_{1}, \ldots, g_{k} \in \Omega$ be such that $g_{i}-g_{j} \in \Omega$ for all $i \neq j$.

Then for any invertible weakly mixing system $(X, \mathcal{B}, \mu, T)$ and any $f_{1}, \ldots, f_{k} \in L^{\infty}(X, \mathcal{B}, \mu)$,

$$
\lim _{N \rightarrow \infty}\left\|\frac{1}{N} \sum_{n=1}^{N} T^{\left[g_{1}(n)\right]} f_{1} \cdots T^{\left[g_{k}(n)\right]} f_{k}-\prod_{i=1}^{k} \int f_{i}\right\|_{2}=0 .
$$

Theorems A and B (as well as Theorem 5.13 in Section 5) are proved by PET-induction (Polynomial Exhaustion Technique) which was used in [2] to prove Theorem 1.3 and which is based on the repeated application of a Hilbert space version of the van der Corput difference theorem. (See the Remark after Theorem 1.5 above and Theorem 4.1 below). However, due to the fact that Theorems A and B deal with much more general classes of functions than Theorem 1.3, there arise many technical difficulties in the course of the implementation of PETinduction. Some of these difficulties are related to the fact that the family $\{[g(n)] \mid g \in \mathcal{G}\}$ is not closed with respect to taking differences, and, in order to deal with the naturally appearing differences of the form $[g(n+h)]-[g(n)]$ one has to modify the van der Corput difference theorem so that it may be applicable to piecewise versions of functions from $\mathcal{G}$. Another natural difficulty is related to the fact that the Fejér functions constituting the family $\mathcal{F}$ are, in a way, too slow, and in order to treat, for example, averages of the form $\frac{1}{N} \sum_{n=1}^{N} T^{\left[g_{1}(n)\right]} f_{1} \cdots T^{\left[g_{k}(n)\right]} f_{k}$, where $g_{i} \in \mathcal{F}$, one has to introduce a change of variables which works only under the additional assumption that certain ratios of functions we deal with have good behavior, (see the Mproperty, Def. 2.8 below). Finally, one more difficulty which one has to overcome has to do with the fact that not only the functions $g_{i}$ appearing in the formulations of Theorems A and $\mathrm{B}$, but also various additional families of functions which emerge at various stages of the inductive procedure, have to have different rates of growth. (This condition cannot be avoided since otherwise one would not get the limit of the averages in question to be that of the form $\left.\prod_{i=1}^{k} \int f_{i}\right)$. While in Theorem 1.3 the distinct growth rates are guaranteed by the condition $p_{i}(n)-p_{j}(n) \not \equiv$ const for $i \neq j$, the situation with the class $\mathcal{G}$ is much more delicate and in order to make the induction working one has to do quite a bit of preliminary technical work.

It is of interest to know under which (additional) conditions Theorems A and B can be extended to general ergodic (rather than weakly mixing) measure-preserving systems. One of the reasons to care about this issue is the fact that it would open interesting possibilities of applications to combinatorial number theory. A partial result in this direction is obtained in Section 7:

Theorem 7.3 Let $g_{1}, g_{2}, \ldots, g_{k} \in \mathcal{F}$ be such that $\lim _{x \rightarrow \infty} \frac{g_{i+1}(x)}{g_{i}(x)}=0$ and the ratio $\frac{g_{i+1}^{\prime}(x)}{g_{i}^{\prime}(x)}$ is eventually monotone, $i=1, \ldots, k-1$. Then for any invertible ergodic system $(X, \mathcal{B}, \mu, T)$ and any $f_{1}, \ldots, f_{k} \in L^{\infty}(X, \mathcal{B}, \mu)$,

$$
\lim _{N \rightarrow \infty}\left\|\frac{1}{N} \sum_{n=1}^{N} T^{\left[g_{1}(n)\right]} f_{1} \cdots T^{\left[g_{k}(n)\right]} f_{k}-\prod_{i=1}^{k} \int f_{i}\right\|_{2}=0
$$


One of the corollaries of Theorem 7.3 which are obtained is the following result.

Corollary 7.6 Let $E \subset \mathbf{N}$ be a subset with $\bar{d}(E)>0$, and let $g_{1}, g_{2}, \ldots, g_{k} \in \mathcal{F}$. Under the same assumptions on $g_{1}, g_{2}, \ldots, g_{k}$ as in Theorem 7.3 one has

$$
\underline{d}\left(\left\{n \in \mathbf{N} \mid \bar{d}\left(E \cap\left(E-\left[g_{1}(n)\right]\right) \cap \cdots \cap\left(E-\left[g_{k}(n)\right]\right)\right) \geq(\bar{d}(E))^{k+1}\right\}\right)>0
$$

The following examples indicate the diversity of possible choices for functions $g$ which satisfy Theorem 7.3 and Corollary 7.6.

- $g_{1}(x)=x^{\alpha_{1}}+x^{\alpha_{2}}, g_{2}(x)=x^{\alpha_{2}}(2+\cos \sqrt{\log x}), g_{3}(x)=x^{\alpha_{3}} \log x, g_{4}(x)=x^{\alpha_{4}}\left(1+\frac{\cos x}{x^{3}}\right)$, where $1>\alpha_{1}>\alpha_{2}>\alpha_{3}>\alpha_{4}>0$.

- $g_{1}(x)=\sqrt{x} \log ^{2} x, g_{2}(x)=\sqrt{x} \log x, g_{3}(x)=\sqrt{x}, g_{4}(x)=\frac{\sqrt{x}}{\log x}$.

The above corollary leads to a natural and rather general conjecture involving functions from the family $\mathcal{T} \cap \mathcal{H}$. (See Conjecture 8.2 in Section 8.)

The paper is organized as follows.

In Section 2 we prepare the ground for the proof of Theorem 4.3 in Section 4 which is the base of the induction procedure in the proofs of Theorems A and B. In particular, we establish some basic properties of functions in class $\mathcal{F}$ which will be needed later in Section 4.

In Section 3 we prove an analogue of Theorem 1.3 for a certain class of piecewise defined functions. This is needed for the proofs of Theorems 4.3, A and B. We also show that the summation method we arrive at after making change of variables is equivalent to the method of Cesàro means. This is needed for the proofs of Theorem 4.3 in Section 4 and Theorem 7.3 in Section 7.

In Section 4 we prove Theorem 4.3.

In Section 5 we take a closer look at the class $\mathcal{T}$ and establish some auxiliary facts to be used in the proofs of Theorems A and B in Section 6. We also prove a theorem for general tempered functions.

In Section 6 we prove Theorems A and B.

In Section 7 we prove, among other things, the aforementioned Theorem 7.3 and Corollary 7.6.

In Section 8 we formulate two natural conjectures which, if true, extend Theorems A, B and 7.3.

\section{Acknowledgement.}

We would like to express our gratitude to the anonymous referee whose thoughtful remarks and constructive criticism were taken into account in the preparation of the revised version. In particular, we owe to the referee the simplified proof of Lemma 2.12 and the presentation of major portion of Section 3 .

\section{$2 \quad$ Fejér functions}

In this section we establish some results about Fejér functions and functions $g$ for which $\lim _{x \rightarrow \infty} \frac{x g^{\prime}(x)}{g(x)}$ exists in $\mathbf{R}$. In particular, we show that functions in $\mathcal{F}$ have the necessary 
properties for the proof of Theorem 4.3 in Section 4 to go through.

In addition to the classes $\mathcal{F}$ and $\mathcal{L}$ (see Definition 1.6), we will use, for $0<\alpha \leq 1$, the class

$$
\mathcal{F}(\alpha)=\left\{g \in \mathcal{F} \mid \lim _{x \rightarrow \infty} \frac{x g^{\prime}(x)}{g(x)}=\alpha\right\} .
$$

Lemma 2.1 Let $\alpha \in \mathbf{R} \backslash\{0\}, g \in C^{\infty}\left(\mathbf{R}^{+}\right)$, and suppose that $\lim _{x \rightarrow \infty} \frac{x g^{\prime}(x)}{g(x)}=\alpha$. If $\alpha>0$, then $\lim _{x \rightarrow \infty}|g(x)|=\infty$, and if $\alpha<0$ then $\lim _{x \rightarrow \infty} g(x)=0$. If $0<\alpha<1$ then $\lim _{x \rightarrow \infty} g^{\prime}(x)=0$.

Proof: Suppose first that $\alpha>0$. Let $0<\epsilon<\alpha$ and let $M=M(\epsilon)$ so that for $x>M$,

$$
\alpha-\epsilon<\frac{x g^{\prime}(x)}{g(x)}<\alpha+\epsilon \text {. }
$$

Then

$$
\int_{M}^{x} \frac{\alpha-\epsilon}{t} d t<\int_{M}^{x} \frac{g^{\prime}(t)}{g(t)} d t<\int_{M}^{x} \frac{\alpha+\epsilon}{t} d t
$$

which implies that

$$
\frac{|g(M)|}{M^{\alpha-\epsilon}} x^{\alpha-\epsilon}<|g(x)|<\frac{|g(M)|}{M^{\alpha+\epsilon}} x^{\alpha+\epsilon} .
$$

This shows that $|g(x)| \rightarrow \infty$ as $x \rightarrow \infty$. Let now $\alpha<0$, and let $g_{1}=1 / g$. Then $\lim _{x \rightarrow \infty} \frac{x g_{1}^{\prime}(x)}{g_{1}(x)}=$ $-\lim _{x \rightarrow \infty} \frac{x g^{\prime}(x)}{g(x)}=-\alpha>0$. Hence, $\lim _{x \rightarrow \infty}\left|g_{1}(x)\right|=\infty$ such that $\lim _{x \rightarrow \infty} g(x)=0$.

If $0<\alpha<1$, let $0<\epsilon<\min \{\alpha, 1-\alpha\}$ such that $\alpha+\epsilon<1$ and therefore by $(6), \lim _{x \rightarrow \infty} \frac{g(x)}{x}=0$. Hence, by (5), $\left|g^{\prime}(x)\right|<(\alpha+\epsilon) \frac{|g(x)|}{x} \rightarrow 0$.

Lemma 2.2 Let $\alpha>0, g \in C^{\infty}\left(\mathbf{R}^{+}\right), g>0$, and suppose that $\lim _{x \rightarrow \infty} \frac{x g^{\prime \prime}(x)}{g^{\prime}(x)}=\alpha-1$. Then $\lim _{x \rightarrow \infty} \frac{x g^{\prime}(x)}{g(x)}=\alpha$ and $\lim _{x \rightarrow \infty} g(x)=\infty$. If $0<\alpha<1$ or $\alpha=1$ and $g^{\prime}(x)$ goes monotonically to 0 , then $g \in \mathcal{F}(\alpha)$.

Proof: $\quad$ Let $0<\epsilon<\alpha$ and let $M=M(\epsilon)$ so that for $x>M$,

$$
\alpha-1-\epsilon<\frac{x g^{\prime \prime}(x)}{g^{\prime}(x)}<\alpha-1+\epsilon \text {. }
$$

Then

$$
\int_{M}^{x} \frac{\alpha-1-\epsilon}{t} d t<\int_{M}^{x} \frac{g^{\prime \prime}(t)}{g^{\prime}(t)} d t<\int_{M}^{x} \frac{\alpha-1+\epsilon}{t} d t
$$

which implies that

$$
\frac{\left|g^{\prime}(M)\right|}{M^{\alpha-1-\epsilon}} x^{\alpha-1-\epsilon}<\left|g^{\prime}(x)\right|<\frac{\left|g^{\prime}(M)\right|}{M^{\alpha-1+\epsilon}} x^{\alpha-1+\epsilon} .
$$

This shows that $\lim _{x \rightarrow \infty} x\left|g^{\prime}(x)\right|=\infty$. Let $c_{1}=\frac{\left|g^{\prime}(M)\right|}{M^{\alpha-1-\epsilon}}$ and $c_{2}=\frac{\left|g^{\prime}(M)\right|}{M^{\alpha-1+\epsilon}}$. By integrating one more time from $M$ to $x>M$, we have, if $g^{\prime}>0$, that

$$
\frac{c_{1}}{\alpha-\epsilon}\left(x^{\alpha-\epsilon}-M^{\alpha-\epsilon}\right)<g(x)-g(M)<\frac{c_{2}}{\alpha+\epsilon}\left(x^{\alpha+\epsilon}-M^{\alpha+\epsilon}\right)
$$


which shows that $\lim _{x \rightarrow \infty} g(x)=\infty$. Similarly, if $g^{\prime}<0$, then $g<0$, contradicting our assumptions. Now, by l'Hopital's rule, we have $\lim _{x \rightarrow \infty} \frac{x g^{\prime}(x)}{g(x)}=1+\lim _{x \rightarrow \infty} \frac{x g^{\prime \prime}(x)}{g^{\prime}(x)}=\alpha$. Hence, if $\alpha=1$ and $g^{\prime}(x)$ goes monotonically to 0 , then $g \in \mathcal{F}(1)$.

Suppose now that $0<\alpha<1$. Then by $(7), \lim _{x \rightarrow \infty} g^{\prime}(x)=0$ and since $\lim _{x \rightarrow \infty} \frac{x g^{\prime \prime}(x)}{g^{\prime}(x)}=$ $\alpha-1<0$ it follows that $g^{\prime \prime}(x)<0$ for sufficiently large $x$. Hence $g^{\prime}(x)$ goes monotonically to 0 . This shows that $g \in \mathcal{F}(\alpha)$.

Lemma 2.3 Let $g \in C^{\infty}\left(\mathbf{R}^{+}\right)$and $\alpha \in \mathbf{R}$ such that $\lim _{x \rightarrow \infty} \frac{x g^{\prime}(x)}{g(x)}=\alpha$. Let $c \in \mathbf{R}$. Then $\lim _{x \rightarrow \infty} \frac{g(x+c)}{g(x)}=1$.

Proof: Let first $0<\alpha<1$. By Lemma 2.1, $\lim _{x \rightarrow \infty}|g(x)|=\infty$ and $\lim _{x \rightarrow \infty} g^{\prime}(x)=0$. Hence, by the mean value theorem, there exists $z_{x} \in(x, x+c)$ such that

$$
\lim _{x \rightarrow \infty} \frac{g(x+c)}{g(x)}=\lim _{x \rightarrow \infty} \frac{g(x)+g^{\prime}\left(z_{x}\right) c}{g(x)}=1 .
$$

If $\alpha \notin(0,1)$ then let $\beta \in \mathbf{R}$ such that $0<\alpha-\beta<1$ and let $g_{1}=g / x^{\beta}$. Then $\lim _{x \rightarrow \infty} \frac{x g_{1}^{\prime}(x)}{g_{1}(x)}=$ $\alpha-\beta$ and hence,

$$
\lim _{x \rightarrow \infty} \frac{g(x+c)}{g(x)}=\lim _{x \rightarrow \infty} \frac{g_{1}(x+c)}{g_{1}(x)} \frac{(x+c)^{\beta}}{x^{\beta}}=1 .
$$

If $g \in C^{\infty}\left(\mathbf{R}^{+}\right)$is a positive function such that $g^{\prime}>0$ and $g^{\prime}$ decreases to 0 , let

$$
\phi(n)=|\{m \in \mathbf{N} \mid n=[g(m)]\}| \quad \text { and } \quad \Phi(n)=\sum_{k=0}^{n} \phi(k) .
$$

When making a change of variables $m=[g(n)]$ in the expression $\frac{1}{N} \sum_{n=1}^{N} T^{\left[g_{1}(n)\right]} f_{1} \cdots T^{\left[g_{k}(n)\right]} f_{k}$, where $g_{i} \in \mathcal{F}$ and $g=g_{1}$, then $\phi(m)$ will be the weights one gets in the new expression. Since $g^{\prime}(x) \rightarrow 0$ as $x \rightarrow \infty, \phi(m) \rightarrow \infty$ as $m \rightarrow \infty$. However, if $g$ is not sufficiently slow, $\phi(m)$ does not need to be an increasing function. This is the case when $g(x)=x^{3 / 4}$ since then there are infinitely many $k$ for which $\phi(k+1)=\phi(k)-1$.

When $f$ is a real-valued function on $\mathbf{R}$ we will use the notation $\Delta f(x)=f(x+1)-f(x)$.

Lemma 2.4 Let $g \in C^{\infty}\left(\mathbf{R}^{+}\right)$be positive such that $g^{\prime}>0$ and $g^{\prime}$ decreases to 0 , and let $\phi$ be defined by (8). Then for $n \in \mathbf{N}, \phi(n)=\Delta g^{-1}(n)+a_{n}$, where $a_{n} \in(-1,1)$ and $\Delta g^{-1}(n)$ increases to infinity as $n \rightarrow \infty$.

Proof: $\quad$ Since $\phi(n)$ is the number of positive integers in the interval $\left[g^{-1}(n), g^{-1}(n+1)\right)$, the first statement follows. Let $f(x)=g^{-1}(x)$. By the mean value theorem, $\Delta f(x)=f^{\prime}\left(z_{x}\right)$, where $x<z_{x}<x+1$. Since $f^{\prime}(x)=\frac{1}{g^{\prime}(f(x))}$ increases to $\infty$ as $x \rightarrow \infty, \Delta f(n) \leq \Delta f(n+1)$ and $\Delta f(n) \rightarrow \infty$ as $n \rightarrow \infty$. 
Lemma 2.5 Let $g$ be a positive Fejér function and let $\phi$ and $\Phi$ be defined by (8). Then $\lim _{n \rightarrow \infty} \frac{\phi(n)}{\Phi(n)}=0$. If $0<\alpha \leq 1$ and $g \in \mathcal{F}(\alpha)$ then $\lim _{n \rightarrow \infty} \phi(n) g^{\prime}\left(g^{-1}(n)\right)=1$ and $\lim _{n \rightarrow \infty} \frac{n \phi(n)}{\Phi(n)}=\frac{1}{\alpha}$.

Proof: Let first $g$ be any positive Fejér function, and let $f(x)=g^{-1}(x)$. By Lemma 2.4 and the mean value theorem, $\phi(n)=f(n+1)-f(n)+a_{n}=f^{\prime}\left(z_{n}\right)+a_{n} \leq f^{\prime}(n+1)+1$, where $a_{n} \in(-1,1)$ and $n \leq z_{n} \leq n+1$. Also, $\Phi(n)=\sum_{k=0}^{n} \phi(k)=|\{m \mid 0 \leq[g(m)] \leq n\}|=\mid\{m \mid$ $0 \leq g(m)<n+1\} \mid=f(n+1)-b_{n}, 0<b_{n} \leq 2$. Hence, $0 \leq \lim _{n \rightarrow \infty} \frac{\phi(n)}{\Phi(n)} \leq \lim _{n \rightarrow \infty} \frac{f^{\prime}(n+1)+1}{f(n+1)-b_{n}}=$ $\lim _{n \rightarrow \infty} \frac{1}{f(n+1) g^{\prime}(f(n+1))}=0$. Suppose now that for some $0<\alpha \leq 1, g \in \mathcal{F}(\alpha)$. Since $\lim _{x \rightarrow \infty} \frac{x f^{\prime}(x)}{f(x)}=\lim _{y=f(x) \rightarrow \infty} \frac{g(y)}{y g^{\prime}(y)}=1 / \alpha, \lim _{x \rightarrow \infty} \frac{x f^{\prime \prime}(x)}{f^{\prime}(x)}=1 / \alpha-1$, and $\lim _{n \rightarrow \infty} \frac{n}{f(n)}=$ $\lim _{n \rightarrow \infty} \frac{1}{f^{\prime}(n)}=\lim _{n \rightarrow \infty} g^{\prime}(f(n))=0$, we have by Lemma 2.3, $\lim _{n \rightarrow \infty} \phi(n) g^{\prime}\left(g^{-1}(n)\right)=$ $\lim _{n \rightarrow \infty} \frac{f^{\prime}\left(z_{n}\right)+a_{n}}{f^{\prime}(n)}=1$ and

$$
\lim _{n \rightarrow \infty} \frac{n \phi(n)}{\Phi(n)}=\lim _{n \rightarrow \infty} \frac{n\left(f^{\prime}\left(z_{n}\right)+a_{n}\right)}{f(n+1)-b_{n}}=\lim _{n \rightarrow \infty} \frac{n f^{\prime}(n)}{f(n)}=\frac{1}{\alpha} .
$$

Remark: If $p_{n}$ is an increasing sequence of positive real numbers and $P_{n}=\sum_{k=0}^{n} p_{k}$, then the property that the sequence $\frac{n p_{n}}{P_{n}}$ is bounded, is the criteria for that the summation method of weighted means, also called a Riesz method $\left(R, p_{n}\right)$, is equivalent to the Cesàro method, see Theorem 3.5 below. Note that if $g$ is a Fejér function with $\lim _{x \rightarrow \infty} \frac{x g^{\prime}(x)}{g(x)}=0$ so that $g \notin \mathcal{F}$, then $\lim _{n \rightarrow \infty} \frac{n \phi(n)}{\Phi(n)}=\infty$ but $\lim _{n \rightarrow \infty} \frac{\phi(n)}{\Phi(n)}=0$. This is the case for $g(x)=\log ^{2} x$ for which $f(x)=g^{-1}(x)=e^{\sqrt{x}}$.

Lemma 2.6 Let $g_{1}, g_{2} \in C^{\infty}\left(\mathbf{R}^{+}\right), g_{1}, g_{2}>0$ and $\alpha_{1}, \alpha_{2} \in \mathbf{R}$. Suppose that $\lim _{x \rightarrow \infty} \frac{x g_{i}^{\prime}(x)}{g_{i}(x)}=$ $\alpha_{i}, i=1,2$. If $\frac{g_{2}(x)}{g_{1}(x)}$ is bounded, then $\alpha_{1} \geq \alpha_{2}$. Conversely, if $\alpha_{1}>\alpha_{2}$ and $\lim _{x \rightarrow \infty} \frac{x g_{i}^{\prime \prime}(x)}{g_{i}^{\prime}(x)}$ exists, $i=1,2$, then $\lim _{x \rightarrow \infty} \frac{g_{2}(x)}{g_{1}(x)}=\lim _{x \rightarrow \infty} \frac{g_{2}^{\prime}(x)}{g_{1}^{\prime}(x)}=0$ and $\frac{g_{2}(x)}{g_{1}(x)}$ and $\frac{g_{2}^{\prime}(x)}{g_{1}^{\prime}(x)}$ are eventually monotone.

Proof: $\quad$ Let $g=g_{2} / g_{1}$. Then $\lim _{x \rightarrow \infty} \frac{x g^{\prime}(x)}{g(x)}=\lim _{x \rightarrow \infty} \frac{x\left(\frac{g_{1} g_{2}^{\prime}-g_{1}^{\prime} g_{2}}{g_{1}^{2}}\right)}{g_{2} / g_{1}}=\lim _{x \rightarrow \infty}\left(\frac{x g_{2}^{\prime}}{g_{2}}-\frac{x g_{1}^{\prime}}{g_{1}}\right)=$ $\alpha_{2}-\alpha_{1}$.

Suppose that $g(x)$ is bounded. Then $\alpha_{2} \leq \alpha_{1}$ by Lemma 2.1 .

Suppose now that $\alpha_{1}>\alpha_{2}$ and that $\lim _{x \rightarrow \infty} \frac{x g_{i}^{\prime \prime}(x)}{g_{i}^{\prime}(x)}$ exists such that $\lim _{x \rightarrow \infty} \frac{x g_{i}^{\prime \prime}(x)}{g_{i}^{\prime}(x)}=\alpha_{i}-1$, $i=1,2$. Then by Lemma $2.1, \lim _{x \rightarrow \infty} g(x)=0$ and if $\tilde{g}=\frac{g_{2}^{\prime}}{g_{1}^{\prime}}$ so that $\lim _{x \rightarrow \infty} \frac{x \tilde{g}^{\prime}(x)}{\tilde{g}(x)}=$ $\lim _{x \rightarrow \infty}\left(\frac{x g_{2}^{\prime \prime}}{g_{2}^{\prime}}-\frac{x g_{1}^{\prime \prime}}{g_{1}^{\prime}}\right)=\alpha_{2}-\alpha_{1}<0$, then $\lim _{x \rightarrow \infty} \tilde{g}(x)=0$. Also, this shows that $g^{\prime}<0$ and $\tilde{g}^{\prime}<0$ so that $\frac{g_{2}(x)}{g_{1}(x)}$ and $\frac{g_{2}^{\prime}(x)}{g_{1}^{\prime}(x)}$ are eventually monotone.

Lemma 2.7 Let $g_{1}, g_{2}$ be positive Fejér functions such that there exists $\gamma \in \mathbf{R}$ with $\lim _{x \rightarrow \infty} \frac{g_{2}(x)}{g_{1}(x)}=$ $\lim _{x \rightarrow \infty} \frac{g_{2}^{\prime}(x)}{g_{1}^{\prime}(x)}=\gamma$. Let $\hat{g}_{2}=g_{2} \circ g_{1}^{-1}$. Then there exist a finite subset $C \subset \mathbf{Z}$ and a sequence 
$c_{n} \in C, n=1,2, \ldots$, such that

$$
\left[g_{2}(n)\right]=\left[\hat{g}_{2}\left(\left[g_{1}(n)\right]\right)\right]+c_{n}, \quad n \in \mathbf{N} .
$$

If $\gamma \neq 0$ then $\hat{g}_{2} \in \mathcal{L}$. If $\gamma=0$ and for some $1 \geq \alpha_{1} \geq \alpha_{2}>0, g_{1} \in \mathcal{F}\left(\alpha_{1}\right), g_{2} \in \mathcal{F}\left(\alpha_{2}\right)$ are such that $\frac{g_{2}^{\prime}(x)}{g_{1}^{\prime}(x)}$ is eventually monotone, then $\hat{g}_{2} \in \mathcal{F}\left(\frac{\alpha_{2}}{\alpha_{1}}\right)$, and $c_{n}=0$ for a set of $n$ of density 1.

Proof: Suppose that $\lim _{x \rightarrow \infty} \frac{g_{2}^{\prime}(x)}{g_{1}^{\prime}(x)}=\gamma \in \mathbf{R}$. By the mean value theorem there exists $z_{n}$, where $\left[g_{1}(n)\right] \leq z_{n} \leq g_{1}(n)$, such that

$$
g_{2}(n)-\hat{g}_{2}\left(\left[g_{1}(n)\right]\right)=\hat{g}_{2}\left(g_{1}(n)\right)-\hat{g}_{2}\left(\left[g_{1}(n)\right]\right)=\hat{g}_{2}^{\prime}\left(z_{n}\right)\left\{g_{1}(n)\right\} .
$$

Since $\lim _{x \rightarrow \infty} \hat{g}_{2}^{\prime}(x)=\lim _{x \rightarrow \infty} \frac{g_{2}^{\prime}\left(g_{1}^{-1}(x)\right)}{g_{1}^{\prime}\left(g_{1}^{-1}(x)\right)}=\gamma, g_{2}(n)-\hat{g}_{2}\left(\left[g_{1}(n)\right]\right)$ is bounded and, hence, there exists a finite set $C \subset \mathbf{Z}$ such that $\left[g_{2}(n)\right]-\left[\hat{g}_{2}\left(\left[g_{1}(n)\right]\right)\right]=c_{n} \in C$ for all $n \in \mathbf{N}$.

If $\gamma \neq 0$ then $\hat{g}_{2} \in \mathcal{L}$ since $\lim _{x \rightarrow \infty} \hat{g}_{2}^{\prime}(x) \neq 0$.

Suppose now that $\gamma=0$ and that for some $1 \geq \alpha_{1} \geq \alpha_{2}>0, g_{1} \in \mathcal{F}\left(\alpha_{1}\right), g_{2} \in \mathcal{F}\left(\alpha_{2}\right)$. Since we assume that both $g_{1}^{-1}(x)$ and $\frac{g_{2}^{\prime}(x)}{g_{1}^{\prime}(x)}$ are eventually monotone functions, $\hat{g}_{2}^{\prime}(x)=\frac{g_{2}^{\prime}\left(g_{1}^{-1}(x)\right)}{g_{1}^{\prime}\left(g_{1}^{-1}(x)\right)}$ goes monotonically to 0 . We also have that $\lim _{x \rightarrow \infty} \frac{x \hat{g}_{2}^{\prime}(x)}{\hat{g}_{2}(x)}=\lim _{x \rightarrow \infty} \frac{g_{1}\left(g_{1}^{-1}(x)\right)}{g_{2}\left(g_{1}^{-1}(x)\right)} \frac{g_{2}^{\prime}\left(g_{1}^{-1}(x)\right)}{g_{1}^{\prime}\left(g_{1}^{-1}(x)\right)}=$ $\lim _{y=g_{1}^{-1}(x) \rightarrow \infty} \frac{g_{1}(y)}{y g_{1}^{\prime}(y)} \frac{y g_{2}^{\prime}(y)}{g_{2}(y)}=\frac{\alpha_{2}}{\alpha_{1}} \leq 1$, and that $\lim _{x \rightarrow \infty} \frac{x \hat{g}_{2}^{\prime \prime}(x)}{\hat{g}_{2}^{\prime}(x)}=\lim _{y \rightarrow \infty} \frac{g_{1}}{y g_{1}^{\prime}}\left(\frac{y g_{2}^{\prime \prime}}{g_{2}^{\prime}}-\frac{y g_{1}^{\prime \prime}}{g_{1}^{\prime}}\right)=$ $\frac{\alpha_{2}}{\alpha_{1}}-1$. Hence, by Lemma $2.2, \hat{g}_{2} \in \mathcal{F}\left(\frac{\alpha_{2}}{\alpha_{1}}\right)$.

Since $\hat{g}_{2}^{\prime}$ is decreasing to 0 , it follows from $(9)$ that $0 \leq g_{2}(n)-\hat{g}_{2}\left(\left[g_{1}(n)\right]\right) \rightarrow 0$. Hence, $C=\{0,1\}$. Let

$$
A=\left\{n \in \mathbf{N} \mid c_{n}=1\right\}=\left\{n \in \mathbf{N} \mid\left[g_{2}(n)\right]=\left[g_{2} \circ g_{1}^{-1}\left(\left[g_{1}(n)\right]\right)\right]+1\right\} .
$$

We will show that $A$ has density 0 . For $i=1,2$ and $m \in \mathbf{N}$ let $I_{i}(m)=\left[g_{i}^{-1}(m), g_{i}^{-1}(m+1)\right)$. Since $x \in I_{i}(m)$ if and only if $\left[g_{i}(x)\right]=m$, we have $n \in I_{1}\left(\left[g_{1}(n)\right]\right) \cap I_{2}\left(\left[g_{2}(n)\right]\right)$ and $g_{1}^{-1}\left(\left[g_{1}(n)\right]\right) \in$ $I_{1}\left(\left[g_{1}(n)\right]\right)$ for all $n \in \mathbf{N}$. Furthermore, $n \in A$ if and only if $g_{1}^{-1}\left(\left[g_{1}(n)\right]\right) \in I_{2}\left(\left[g_{2}(n)\right]-1\right)$. Hence, $n \in A$ if and only if $g_{2}^{-1}\left(\left[g_{2}(n)\right]\right)$ is in the interior of the interval $I_{1}\left(\left[g_{1}(n)\right]\right)$ since if $n \in A$ then $I_{1}\left(\left[g_{1}(n)\right]\right)$ has non-empty intersection with both $I_{2}\left(\left[g_{2}(n)\right]-1\right)$ and $I_{2}\left(\left[g_{2}(n)\right]\right)$. So any $n \in A$ is an element of an interval $I_{1}(k)$ (where $k=\left[g_{1}(n)\right]$ ) which also contains an element $g_{2}^{-1}(m) \leq n$, where $m \in \mathbf{N}$. The number of such intervals $I_{1}(k)$ containing some $n \in A \cap[1, N]$ equals the number of $m$ for which $g_{2}^{-1}(m) \leq N$. Hence, the number of such intervals is $\left[g_{2}(N)\right]$. The length of the interval $I_{1}\left(\left[g_{1}(n)\right]\right), n \leq N$, is less than or equal to $\phi_{1}\left(\left[g_{1}(N)\right]\right)+1$, where $\phi_{1}(k)=\left|\left\{m \in \mathbf{N} \mid\left[g_{1}(m)\right]=k\right\}\right|$, by Lemma 2.4. By Lemma 2.5, $\lim _{n \rightarrow \infty} \phi_{1}(n) g_{1}^{\prime}\left(g_{1}^{-1}(n)\right)=1$. Thus,

$$
\begin{aligned}
d(A) & =\lim _{N \rightarrow \infty} \frac{1}{N}|A \cap[1, N]| \leq \lim _{N \rightarrow \infty} \frac{1}{N}\left[g_{2}(N)\right]\left(\phi_{1}\left(\left[g_{1}(N)\right]\right)+1\right)=\lim _{N \rightarrow \infty} \frac{g_{2}(N)}{N} \frac{1}{g_{1}^{\prime}(N)} \\
& =\lim _{N \rightarrow \infty} \frac{g_{1}(N)}{N g^{\prime}(N)} \frac{g_{2}(N)}{g_{1}(N)}=0 .
\end{aligned}
$$


The following definition of M-property of a set will be needed for Theorem 4.3 below. Since Theorem 4.3 is the base of induction of the proofs of the theorems A and B, this M-property gives restrictions on the functions of higher orders in Theorem B. Note that any finite subset of $\mathcal{G}_{0}$ which has the R-property also has the M-property (see Proposition 5.11 below). Recall that when $G$ is a finite set of functions, we use the notation $G_{\text {diff }}=\left\{g_{2}-g_{1} \mid g_{1}, g_{2} \in G, g_{1} \neq g_{2}\right\}$.

Definition 2.8 Let $G \subset \mathcal{F} \cup \mathcal{L}$ be a finite set for which $G_{\text {diff }} \subset \mathcal{F} \cup \mathcal{L}$. If $0<\alpha \leq 1$, let $G(\alpha)=G \cap \mathcal{F}(\alpha)$. $G$ is said to have the M-property ( $M$ for monotonicity) if for all $0<\alpha \leq 1$ and all pairs $\psi_{1}, \psi_{2} \in\left(G \cup G_{\text {diff }}\right) \cap \mathcal{F}$ such that either $\psi_{1}, \psi_{2} \in G(\alpha) \cup G(\alpha)_{\text {diff }}$ or $\psi_{1}, \psi_{2} \in(G \cap \mathcal{L})_{\text {diff }}$, we have

(a) $L=\lim _{x \rightarrow \infty} \frac{\psi_{1}^{\prime}(x)}{\psi_{2}^{\prime}(x)}$ exists in $\mathbf{R} \cup\{ \pm \infty\}$ and

(b) if $L \in\{0, \pm \infty\}$ and $f=\frac{\psi_{1}^{\prime}}{\psi_{2}^{\prime}}$ then $f$ is eventually monotone and if $\lim _{x \rightarrow \infty} \frac{x f^{\prime}(x)}{f(x)}=0$ then $\lim _{x \rightarrow \infty} \frac{x f^{\prime \prime}(x)}{f^{\prime}(x)}$ exists.

Remark: It is not clear if the condition $\lim _{x \rightarrow \infty} \frac{x f^{\prime \prime}(x)}{f^{\prime}(x)}$ exists, is automatically satisfied when the other conditions are satisfied. However, if the limit exists then the value is -1 according to the following lemma.

Lemma 2.9 Let $f \in C^{\infty}\left(\mathbf{R}^{+}\right)$such that $\lim _{x \rightarrow \infty} \frac{x f^{\prime}(x)}{f(x)}=0, \lim _{x \rightarrow \infty} f(x) \in\{0, \pm \infty\}$ and $\lim _{x \rightarrow \infty} \frac{x f^{\prime \prime}(x)}{f^{\prime}(x)}$ exists. Then $\lim _{x \rightarrow \infty} \frac{x f^{\prime \prime}(x)}{f^{\prime}(x)}=-1$.

Proof: $\quad$ Suppose first that $\lim _{x \rightarrow \infty} f(x)=0$. Since $\lim _{x \rightarrow \infty} \frac{x f^{\prime}(x)}{f(x)}=0, \lim _{x \rightarrow \infty} x f^{\prime}(x)=0$, such that by l'Hopital's rule, $0=\lim _{x \rightarrow \infty} \frac{x f^{\prime}(x)}{f(x)}=\lim _{x \rightarrow \infty} \frac{x f^{\prime \prime}(x)}{f^{\prime}(x)}+1$. Hence, $\lim _{x \rightarrow \infty} \frac{x f^{\prime \prime}(x)}{f^{\prime}(x)}=$ -1 .

Suppose now that $\lim _{x \rightarrow \infty} f(x)=\infty$, and let $f_{1}=1 / f$. Since $\lim _{x \rightarrow \infty} \frac{x f_{1}^{\prime}(x)}{f_{1}(x)}=-\lim _{x \rightarrow \infty} \frac{x f^{\prime}(x)}{f(x)}=$ 0 and $\lim _{x \rightarrow \infty} \frac{x f_{1}^{\prime \prime}(x)}{f_{1}^{\prime}(x)}=\lim _{x \rightarrow \infty}\left(\frac{x f^{\prime \prime}(x)}{f^{\prime}(x)}-2 \frac{x f^{\prime}(x)}{f(x)}\right)=-1$, we are done.

Lemma 2.10 Let $\lambda<0$ and let $f_{1}, f_{2} \in C^{\infty}\left(\mathbf{R}^{+}\right)$such that $\lim _{x \rightarrow \infty} f_{1}(x)=0, f_{1}^{\prime}<0$ and such that $\lim _{x \rightarrow \infty} \frac{x f_{1}^{\prime}(x)}{f_{1}(x)}=\lim _{x \rightarrow \infty} \frac{x f_{2}^{\prime}(x)}{f_{2}(x)}=0$ and $\lim _{x \rightarrow \infty} \frac{x f_{1}^{\prime \prime}(x)}{f_{1}^{\prime}(x)}=-1$. Let $\phi=f_{1}+x^{\lambda} f_{2}$. Then $\phi^{\prime}<0$.

Proof: We have

$$
\phi^{\prime}=f_{1}^{\prime}+x^{\lambda-1} f_{2}\left(\lambda+\frac{x f_{2}^{\prime}}{f_{2}}\right)=f_{1}^{\prime}\left(1+\frac{1}{x^{1-\lambda} f_{1}^{\prime} f_{2}^{-1}}\left(\lambda+\frac{x f_{2}^{\prime}}{f_{2}}\right)\right) .
$$

Let $\theta=x^{1-\lambda} f_{1}^{\prime} f_{2}^{-1}$. Then $\frac{x \theta^{\prime}}{\theta}=1-\lambda+\frac{x f_{1}^{\prime \prime}}{f_{1}^{\prime}}-\frac{x f_{2}^{\prime}}{f_{2}} \rightarrow-\lambda>0$. Hence, by Lemma 2.1, $\lim _{x \rightarrow \infty}|\theta(x)|=\infty$, and therefore $\phi^{\prime}$ has the same sign as $f_{1}^{\prime}$. 
Proposition 2.11 Let $g_{1}, \ldots, g_{k} \in \mathcal{F}$ be such that for $i \neq j, g_{i}-g_{j} \in \mathcal{F}$, and such that $\left\{g_{1}, \ldots, g_{k}\right\}$ has the M-property (Definition 2.8). Suppose that for each $i, \frac{g_{i}(x)}{g_{1}(x)}$ is bounded. Let $\hat{g}_{i}(x)=g_{i} \circ g_{1}^{-1}(x)$ if $g_{1}>0$ and let $\hat{g}_{i}(x)=g_{i} \circ\left(-g_{1}\right)^{-1}(x)$ if $g_{1}<0$. Then for each $i$ and $j \neq i, \hat{g}_{i}, \hat{g}_{j}-\hat{g}_{i} \in \mathcal{F} \cup \mathcal{L}$ and $\left\{\hat{g}_{1}, \ldots, \hat{g}_{k}\right\} \subset \mathcal{F} \cup \mathcal{L}$ has the M-property.

Proof: $\quad$ Suppose that $0<\alpha_{i} \leq 1, i=1, \ldots, k$, are such that $\lim _{x \rightarrow \infty} \frac{x g_{i}^{\prime}}{g_{i}}=\alpha_{i}$. Since $\frac{g_{i}}{g_{1}}$ is bounded, $\alpha_{i} \leq \alpha_{1}$ for all $i$ by Lemma 2.6. Suppose that $g_{1}>0$. If $\alpha_{i}<\alpha_{1}$ then by Lemma 2.6 the pair $g_{1}, g_{i}$ satisfies the conditions in Lemma 2.7 in order that $\hat{g}_{i} \in \mathcal{F}\left(\frac{\alpha_{i}}{\alpha_{1}}\right)$. If $\alpha_{i}=\alpha_{1}$ then the M-property and l'Hopital's rule assure that the same conditions are satisfied. Hence, $\hat{g}_{i} \in \mathcal{F}\left(\frac{\alpha_{i}}{\alpha_{1}}\right) \cup \mathcal{L}$ for all $i$. Similarly, by Lemma 2.6, the M-property and Lemma 2.7, $\hat{g}_{i}-\hat{g}_{j} \in \mathcal{F} \cup \mathcal{L}$ if $i \neq j$ and $g_{i}-g_{j} \in \mathcal{F}(\beta)$, where $\beta<\alpha_{1}$, or $g_{i}, g_{j} \in \mathcal{F}\left(\alpha_{1}\right)$. Suppose now that $g_{i} \in \mathcal{F}\left(\alpha_{1}\right)$ and $g_{j} \in \mathcal{F}(\beta)$ for some $\beta<\alpha_{1}$ and that $\frac{g_{i}^{\prime}}{g_{1}^{\prime}}$ goes monotonically to 0 . We need to show that also $\frac{g_{i}^{\prime}-g_{j}^{\prime}}{g_{1}^{\prime}}$ goes monotonically to 0 . Let $f_{i}=\frac{g_{i}^{\prime}}{g_{1}^{\prime}}$ and $f_{j}=\frac{g_{j}^{\prime}}{g_{1}^{\prime}} x^{\alpha_{1}-\beta}$. Then $\lim _{x \rightarrow \infty} \frac{x f_{i}^{\prime}}{f_{i}}=\lim _{x \rightarrow \infty} \frac{x f_{j}^{\prime}}{f_{j}}=0$. Since $f_{i}$ goes to 0 , it follows by the M-property and Lemma 2.9 that $\lim _{x \rightarrow \infty} \frac{x f_{i}^{\prime \prime}}{f_{i}^{\prime}}=-1$. Hence, by Lemma $2.10, \frac{g_{i}^{\prime}-g_{j}^{\prime}}{g_{1}^{\prime}}=f_{i}-x^{\beta-\alpha_{1}} f_{j}$ goes monotonically to 0 , and by Lemma $2.7, \hat{g}_{i}-\hat{g}_{j} \in \mathcal{F}(1)$.

It is left to prove that $\hat{G}$ has the M-property. Let $0<\alpha \leq 1, G(\alpha)=\left\{g_{1}, \ldots, g_{k}\right\} \cap \mathcal{F}(\alpha)$ and $\hat{G}\left(\frac{\alpha}{\alpha_{1}}\right)=\left\{\hat{g}_{1}, \ldots, \hat{g}_{k}\right\} \cap \mathcal{F}\left(\frac{\alpha}{\alpha_{1}}\right)$, and let $\psi_{1}, \psi_{2} \in \hat{G}\left(\frac{\alpha}{\alpha_{1}}\right) \cup \hat{G}\left(\frac{\alpha}{\alpha_{1}}\right)_{\text {diff. }}$. Then there exist $\phi_{1}, \phi_{2} \in G(\alpha) \cup G(\alpha)_{\text {diff }}$ such that $\psi_{i}=\phi_{i} \circ g_{1}^{-1}, i=1,2$, and $\frac{\psi_{1}}{\psi_{2}}$ and $\frac{\psi_{1}^{\prime}}{\psi_{2}^{\prime}}=\frac{\phi_{1}^{\prime} \circ g_{1}^{-1}}{\phi_{2}^{\prime} \circ g_{1}^{-1}}$ have the same asymptotic behavior as the ratios coming from the original functions since $g_{1}$ is monotone. However, we need to prove that if $\theta=\frac{\psi_{1}^{\prime}}{\psi_{2}^{\prime}}$ and $\lim _{x \rightarrow \infty} \theta(x) \in\{0, \pm \infty\}$, then $\lim _{x \rightarrow \infty} \frac{x \theta^{\prime \prime}(x)}{\theta^{\prime}(x)}=-1$. Let $r=\frac{\phi_{1}^{\prime}}{\phi_{2}^{\prime}}$ such that $\theta(x)=r(y)$, where $y=g_{1}^{-1}(x)$. We have $y^{\prime}=\frac{1}{g_{1}^{\prime}(y)}$, $y^{\prime \prime}=-\frac{g_{1}^{\prime \prime}(y)}{\left(g_{1}^{\prime}(y)\right)^{2}} y^{\prime}$ and by the M-property of $G, \lim _{x \rightarrow \infty} \frac{x r^{\prime \prime}}{r^{\prime}}=-1$ so that

$$
\begin{aligned}
\lim _{x \rightarrow \infty} \frac{x \theta^{\prime \prime}(x)}{\theta^{\prime}(x)}=\lim _{x \rightarrow \infty} \frac{x\left(r^{\prime \prime}(y)\left(y^{\prime}\right)^{2}+r^{\prime}(y) y^{\prime \prime}\right)}{r^{\prime}(y) y^{\prime}} & =\lim _{x \rightarrow \infty}\left(\frac{y r^{\prime \prime}(y)}{r^{\prime}(y)} \frac{g_{1}(y)}{y g_{1}^{\prime}(y)}-\frac{g_{1}(y)}{y g_{1}^{\prime}(y)} \frac{y g_{1}^{\prime \prime}(y)}{g_{1}^{\prime}(y)}\right) \\
& =(-1) \frac{1}{\alpha_{1}}-\frac{1}{\alpha_{1}}\left(\alpha_{1}-1\right)=-1 .
\end{aligned}
$$

Lemma 2.12 Let $g \in C^{\infty}\left(\mathbf{R}^{+}\right)$and $\gamma \in \mathbf{R}$ be such that $g^{\prime}(x) \rightarrow \gamma$ as $x \rightarrow \infty$. Let $h \in \mathbf{N}$. Then

$$
[g(n+h)]-[g(n)]=[g(n+h)-g(n)]+b_{h}(n)=[\gamma h]+a_{h}(n)
$$

where $b_{h}(n) \in\{0,1\}$ and there exists $M_{h}$ such that for $n>M_{h}, a_{h}(n) \in\{0, \pm 1\}$. If $\gamma=0$ and $g$ is monotone then $a_{h}(n)=0$ for a set of $n$ of density 1 .

Proof: $\quad$ Since $[x-y]=[x]-[y]-\delta$, where $\delta=0$ if $\{x\} \geq\{y\}$ and 1 otherwise, $b_{h}(n) \in\{0,1\}$. If $\gamma h \notin \mathbf{Z}$ let $0<\epsilon<\frac{1}{h} \min \{\{\gamma h\}, 1-\{\gamma h\}\}$, and if $\gamma h \in \mathbf{Z}$ let $0<\epsilon<\frac{1}{h}$. Since $g^{\prime}(x) \rightarrow \gamma$, there exists $M_{h}$ such that for $x>M_{h},\left|g^{\prime}(x)-\gamma\right|<\epsilon$. Let $n>M_{h}$. Then by the mean value theorem 
there exists $z_{n}, n<z_{n}<n+h$, such that $[g(n+h)-g(n)]=\left[g^{\prime}\left(z_{n}\right) h\right]=\left[\gamma h+\left(g^{\prime}\left(z_{n}\right)-\gamma\right) h\right]$ which equals $[\gamma h]$ if $\gamma h \notin \mathbf{Z}$, or if $\gamma h \in \mathbf{Z}$ and $g^{\prime}\left(z_{n}\right) \geq \gamma$. Otherwise, if $\gamma h \in \mathbf{Z}$ and $g^{\prime}\left(z_{n}\right)<\gamma$ then $[g(n+h)-g(n)]=[\gamma h]-1$. This shows that $a_{h}(n) \in\{0, \pm 1\}$.

Suppose that $\gamma=0$ and that $g>0$. If $\{n \in \mathbf{N} \mid[g(n)]<[g(n+1)]\}=\left\{n_{1}<n_{2}<n_{3}<\cdots\right\}$ then $g\left(n_{k+1}+1\right)-g\left(n_{k}\right) \geq 1$ and by the mean value theorem there exists $n_{k}<z_{k}<n_{k+1}+1$ such that $g\left(n_{k+1}+1\right)-g\left(n_{k}\right)=g^{\prime}\left(z_{k}\right)\left(n_{k+1}+1-n_{k}\right)$. Hence, $n_{k+1}-n_{k} \rightarrow \infty$ as $k \rightarrow \infty$. Thus the set $\left\{n \in \mathbf{N} \mid \exists k, n \leq n_{k}<n+h\right\}$ has zero density. The proof for the case $g<0$ is similar.

\section{$3 \quad$ Weakly mixing systems and summation methods}

In this section we collect some results which will be needed in the next sections. These include some basic facts on weakly mixing systems as well as a result on summation methods in normed linear spaces. From now on we will be assuming that all the vector spaces we work with are over the real scalars. (It is not hard to see that this assumption can be made without loss of generality.)

In the following theorem, the equivalence of the first three conditions is well known and can be found in any basic text on ergodic theory. As for the condition (iv), see [18], [14, p. 96], and $[1]$.

Theorem 3.1 Let $(X, \mathcal{B}, \mu, T)$ be an invertible measure preserving system. Then the following are equivalent.

(i) $T$ is weakly mixing, i.e. for any $A, B \in \mathcal{B}$ one has $\lim _{n \rightarrow \infty} \frac{1}{N} \sum_{n=1}^{N} \mid \mu\left(A \cap T^{-n} B\right)-$ $\mu(A) \mu(B) \mid=0$.

(ii) For all $f, g \in L^{2}(X, \mathcal{B}, \mu), \lim _{N \rightarrow \infty} \frac{1}{N} \sum_{n=1}^{N}\left|\int f T^{n} g d \mu-\int f d \mu \int g d \mu\right|=0$.

(iii) $T \times T$ is weakly mixing.

(iv) For all $f \in L^{2}(X, \mathcal{B}, \mu)$ with $\int f=0$ and any sequence $n_{k}$ of positive lower density,

$$
\lim _{N \rightarrow \infty}\left\|\frac{1}{N} \sum_{k=1}^{N} T^{n_{k}} f\right\|_{2}=0 .
$$

Lemma 3.2 Let $a_{i}(n), i=1, \ldots, k$, be $k$ integer-valued sequences. Suppose that for any weakly mixing system $(X, \mathcal{B}, \mu, T)$ and any $f_{i} \in L^{\infty}(X, \mathcal{B}, \mu), i=1, \ldots, k$, with $\int f_{1} d \mu=0$, we have

$$
\lim _{N \rightarrow \infty}\left\|\frac{1}{N} \sum_{n=1}^{N} T^{a_{1}(n)} f_{1} T^{a_{2}(n)} f_{2} \cdots T^{a_{k}(n)} f_{k}\right\|_{2}=0 .
$$

Then, for any bounded sequence $u_{n}$ of real numbers, for any weakly mixing system $(X, \mathcal{B}, \mu, T)$ and any $f_{i} \in L^{\infty}(X, \mathcal{B}, \mu), i=1, \ldots, k$, with $\int f_{1} d \mu=0$,

$$
\lim _{N \rightarrow \infty}\left\|\frac{1}{N} \sum_{n=1}^{N} u_{n} T^{a_{1}(n)} f_{1} T^{a_{2}(n)} f_{2} \cdots T^{a_{k}(n)} f_{k}\right\|_{2}=0 .
$$


Proof: $\quad$ Suppose that $\left|u_{n}\right|<c$ for all $n$. Let $F_{n}=T^{a_{1}(n)} f_{1} T^{a_{2}(n)} f_{2} \cdots T^{a_{k}(n)} f_{k}$. Then

$$
\left\|\frac{1}{N} \sum_{n=1}^{N} u_{n} F_{n}\right\|_{2}^{2}=\int\left|\frac{1}{N} \sum_{n=1}^{N} u_{n} F_{n}\right|^{2} d \mu \leq \frac{c^{2}}{N^{2}} \sum_{n, m}\left|\int F_{n} F_{m} d \mu\right|
$$

and

$$
\frac{1}{N^{2}} \sum_{n, m}\left|\int F_{n} F_{m} d \mu\right|^{2}=\iint\left|\frac{1}{N} \sum_{n=1}^{N} F_{n}(x) F_{n}(y)\right|^{2} d \mu(x) d \mu(y)
$$

which goes to zero when $N$ tends to infinity, since $T \times T$ is weakly mixing.

Proposition 3.3 Let $\theta_{i j}(n), n \in \mathbf{N}, j=1, \ldots, l, i=1, \ldots, k$, be integer-valued sequences such that for any weakly mixing system $(X, \mathcal{B}, \mu, T)$, any $f_{i} \in L^{\infty}(X, \mathcal{B}, \mu), i=1, \ldots, k$, and any $j \in\{1, \ldots, l\}$ one has

$$
\lim _{N \rightarrow \infty}\left\|\frac{1}{N} \sum_{n=1}^{N} T^{\theta_{1 j}(n)} f_{1} T^{\theta_{2 j}(n)} f_{2} \cdots T^{\theta_{k j}(n)} f_{k}-\prod_{i=1}^{k} \int f_{i} d \mu\right\|_{2}=0 .
$$

Let $\bigcup_{j=1}^{l} C_{j}$ be a partition of $\mathbf{N}$ and let $\theta_{i}(n)=\theta_{i j}(n)$ iff $n \in C_{j}, j=1, \ldots, l$. Then for any weakly mixing system $(X, \mathcal{B}, \mu, T)$ and any $f_{i} \in L^{\infty}(X, \mathcal{B}, \mu), i=1, \ldots, k$, one has

$$
\lim _{N \rightarrow \infty}\left\|\frac{1}{N} \sum_{n=1}^{N} T^{\theta_{1}(n)} f_{1} T^{\theta_{2}(n)} f_{2} \cdots T^{\theta_{k}(n)} f_{k}-\prod_{i=1}^{k} \int f_{i} d \mu\right\|_{2}=0 .
$$

Proof: Let $u_{j}(n)=1$ if $n \in C_{j}$ and 0 otherwise. Then $T^{\theta_{1}(n)} f_{1} T^{\theta_{2}(n)} f_{2} \cdots T^{\theta_{k}(n)} f_{k}=$ $\sum_{j=1}^{k} u_{j}(n) T^{\theta_{1 j}(n)} f_{1} T^{\theta_{2 j}(n)} f_{2} \cdots T^{\theta_{k j}(n)} f_{k}$. The result now follows from the previous lemma.

Note that if for some $g_{i} \in \mathcal{G}, i=1, \ldots, k$, one already knows that

$$
\frac{1}{N} \sum_{n=1}^{N} T^{\left[g_{1}(n)\right]} f_{1} \cdots T^{\left[g_{k}(n)\right]} f_{k} \rightarrow \prod_{i=1}^{k} \int f_{i}
$$

for any weakly mixing system and any $f_{1}, \ldots, f_{k} \in L^{\infty}(X)$, then the same result holds for $\left[g_{i}(n)\right]+c_{i}(n)$ if $c_{i}(n)$ takes on only finitely many values, $i=1, \ldots, k$.

The following simple lemma will be utilized in the proof of Theorem 4.3 in the next section.

Let $y=y(n, h), n, h \geq 1$, be a bounded family of positive numbers. We say that $y$ satisfies the condition $(\mathrm{C})$ if

$$
\lim _{H \rightarrow \infty} \frac{1}{H} \sum_{h=1}^{H} \limsup _{N \rightarrow \infty} \frac{1}{N} \sum_{n=1}^{N} y(n, h)=0 .
$$

Lemma 3.4 Let $y_{1}, \ldots, y_{l}$ be bounded families of positive numbers which satisfy the condition (C). Suppose $y$ is a family such that for all $h$, for all large enough $n, y(n, h) \in\left\{y_{i}(n, h) \mid i=\right.$ $1, \ldots, l\}$. Then $y$ satisfies $(C)$. 
Proof: It is clear that

(i) $\sum_{i=1}^{l} y_{i}$ satisfies the condition $(\mathrm{C})$,

(ii) if $\tilde{y}$ is a family that satisfies (C) and if $y$ is another family such that, for all $h$, for all large enough $n, y(n, h) \leq \tilde{y}(n, h)$, then $y$ satisfies $(\mathrm{C})$.

Let $\tilde{y}=\sum_{i=1}^{l} y_{i}$. Then for all $h, y(n, h) \leq \tilde{y}(n, h)$ for large enough $n$, and hence, $y$ satisfies (C).

The next theorem is an extension of one of the classical results on summation methods for real-valued sequences, (see [16, Theorem 14]), to sequences in normed linear spaces; it will be used in the proofs of Theorems 4.3 and 7.3 below.

Theorem 3.5 Let $p_{n}, n=1,2, \ldots$, be a sequence of positive numbers such that $\lim _{n \rightarrow \infty} p_{n}=$ $\infty$ and such that there exists an increasing sequence $q_{n}$ with $p_{n}-q_{n}$ bounded. Suppose also that $\frac{n p_{n}}{P_{n}} \leq c$ for some $c>0$, where $P_{n}=\sum_{i=1}^{n} p_{i}$. Let $x_{n}, n=1,2, \ldots$, be a bounded sequence in a normed linear space. Then $\lim _{N \rightarrow \infty}\left\|\frac{1}{N} \sum_{n=1}^{N} x_{n}\right\|=0$ if and only if $\lim _{N \rightarrow \infty}\left\|\frac{1}{P_{N}} \sum_{n=1}^{N} p_{n} x_{n}\right\|=$ 0 .

Proof: Let $a_{n}=p_{n}-q_{n}$ and let $d>0$ such that $\left|a_{n}\right|<d$ for all $n$. If $Q_{n}=\sum_{k=1}^{n} q_{n}$ then $\lim _{n \rightarrow \infty} \frac{P_{n}}{Q_{n}}=\lim _{n \rightarrow \infty} \frac{Q_{n}+\sum_{k=1}^{n} a_{k}}{Q_{n}}=1$ since $\lim _{n \rightarrow \infty} q_{n}=\infty$ so that $\left|\frac{1}{Q_{n}} \sum_{k=1}^{n} a_{k}\right| \leq d \frac{n}{Q_{n}} \rightarrow 0$. Hence, there exists $c_{1} \geq c$ such that $\frac{n q_{n}}{Q_{n}}=\frac{P_{n}}{Q_{n}} \frac{n p_{n}}{P_{n}}+\frac{n a_{n}}{Q_{n}} \leq c_{1}$ for all sufficiently large $n$. Since $x_{n}$ is bounded, $\lim _{N \rightarrow \infty}\left\|\frac{1}{P_{N}} \sum_{n=1}^{N} a_{n} x_{n}\right\|=0$. We therefore have that $\lim _{N \rightarrow \infty}\left\|\frac{1}{P_{N}} \sum_{n=1}^{N} p_{n} x_{n}\right\|=0$ if and only if $\lim _{N \rightarrow \infty}\left\|\frac{1}{Q_{N}} \sum_{n=1}^{N} q_{n} x_{n}\right\|=0$.

Suppose that $\lim _{N \rightarrow \infty}\left\|\frac{1}{N} \sum_{n=1}^{N} x_{n}\right\|=0$. Let $\epsilon>0$. There exists $N_{0}$ such that for $N>N_{0}$, $\left\|\sum_{n=1}^{N} x_{n}\right\| \leq N \epsilon$. Let $s_{n}=\sum_{k=n}^{N} x_{k}$. For $N_{0} \leq m \leq N$, we have $\left\|s_{m}\right\| \leq 2 N \epsilon$. We have

$$
\begin{aligned}
\left\|\frac{1}{Q_{N}} \sum_{n=N_{0}}^{N} q_{n} x_{n}\right\| & =\left\|\frac{1}{Q_{N}}\left(\sum_{n=N_{0}}^{N-1} q_{n}\left(s_{n}-s_{n+1}\right)+q_{N} s_{N}\right)\right\| \\
& =\frac{1}{Q_{N}}\left\|\sum_{n=N_{0}+1}^{N}\left(q_{n}-q_{n-1}\right) s_{n}+q_{N_{0}} s_{N_{0}}\right\| \\
& \leq \frac{1}{Q_{N}}\left(\sum_{n=N_{0}+1}^{N}\left(q_{n}-q_{n-1}\right)\left\|s_{n}\right\|+q_{N_{0}}\left\|s_{N_{0}}\right\|\right) \\
& \leq \frac{1}{Q_{N}}(2 N \epsilon)\left(2 q_{N}\right) \leq 4 c_{1} \epsilon .
\end{aligned}
$$

Hence, $\lim \sup _{N \rightarrow \infty}\left\|\frac{1}{Q_{N}} \sum_{n=1}^{N} q_{n} x_{n}\right\| \leq 4 c_{1} \epsilon$.

The other direction is true for any increasing $q_{n}$ and can be proved similarly. 
In order to prove a theorem for Fejér functions which are slower than those in $\mathcal{T}_{0}$ (see Theorem 5.13 below), we need the following theorem which is proved by Lorentz [20, Theorem 7] for real valued sequences $x_{n}$, see also [8, Theorem 2.4.9]. The same proof works for sequences in any normed linear spaces and we include it here to make the paper self-contained.

Theorem 3.6 Let $p_{n}$ be an increasing sequence of real numbers such that $p_{n} \rightarrow \infty$, and such that if $P_{n}=\sum_{k=0}^{n} p_{k}$ then $\lim _{n \rightarrow \infty} \frac{p_{n}}{P_{n}}=0$. If $x_{n}$ is a bounded sequence in a normed linear space such that

$$
\lim _{N \rightarrow \infty}\left\|\frac{1}{N+1} \sum_{n=0}^{N} x_{h+n}\right\|=0 \quad \text { uniformly in } h,
$$

then

$$
\lim _{N \rightarrow \infty}\left\|\frac{1}{P_{N}} \sum_{n=0}^{N} p_{n} x_{n}\right\|=0 .
$$

Proof: $\quad$ Suppose that $c \in \mathbf{R}^{+}$is such that $\left\|x_{k}\right\|<c$ for all $k$. Let $\epsilon>0$ and let $N_{0} \in \mathbf{N}$ such that

$$
\left\|\frac{1}{N_{0}+1} \sum_{n=h}^{h+N_{0}} x_{n}\right\|<\epsilon / 4 \text { for all } h \in \mathbf{N}
$$

and let $n_{0}=n_{0}\left(N_{0}\right)$ such that

$$
\frac{p_{N}}{P_{N}}<\frac{\epsilon}{4 N_{0} c} \text { for all } N>n_{0} .
$$

Let $N>n_{0}$, and for this $N$ let $\tilde{p}_{n}=p_{n}$ if $0 \leq n \leq N$ and let $\tilde{p}_{n}=0$ otherwise. We have

$$
\begin{aligned}
\left\|\frac{1}{P_{N}} \sum_{n=0}^{N} p_{n} x_{n}\right\| \leq & \underbrace{}_{\beta} \underbrace{\frac{1}{P_{N}} \sum_{n=0}^{N} p_{n} x_{n}-\frac{1}{N_{0}+1} \sum_{n=N_{0}}^{N+N_{0}} x_{n} \frac{1}{P_{N}} \sum_{k=n-N_{0}}^{n} \tilde{p}_{k} \|} \| \\
& +\underbrace{\frac{1}{N_{0}+1}\left\|\sum_{n=N_{0}}^{N+N_{0}} x_{n} \frac{1}{P_{N}} \sum_{k=n-N_{0}}^{n} \tilde{p}_{k}-\frac{1}{P_{N}} \sum_{k=0}^{N} p_{k} \sum_{n=k}^{k+N_{0}} x_{n}\right\|}_{\delta} \|
\end{aligned}
$$

where $\delta \leq \epsilon / 4$ by (11),

$$
\begin{aligned}
\gamma & =\frac{1}{N_{0}+1}\left\|\sum_{n=N_{0}}^{N+N_{0}} x_{n} \frac{1}{P_{N}} \sum_{k=n-N_{0}}^{n} \tilde{p}_{k}-\sum_{n=0}^{N+N_{0}} x_{n} \frac{1}{P_{N}} \sum_{k=n-N_{0}}^{n} \tilde{p}_{k}\right\| \\
& =\frac{1}{N_{0}+1}\left\|\sum_{n=0}^{N_{0}-1} x_{n} \frac{1}{P_{N}} \sum_{k=n-N_{0}}^{n} \tilde{p}_{k}\right\| \leq c N_{0} \frac{p_{N}}{P_{N}}<\frac{\epsilon}{4}
\end{aligned}
$$


by (12), and

$$
\begin{aligned}
\beta & =\left\|\frac{1}{P_{N}} \sum_{n=0}^{N_{0}-1} \tilde{p}_{n} x_{n}-\frac{1}{N_{0}+1} \sum_{n=N_{0}}^{N+N_{0}} x_{n} \frac{1}{P_{N}}\left(\sum_{k=n-N_{0}}^{n} \tilde{p}_{k}-\tilde{p}_{n}\left(N_{0}+1\right)\right)\right\| \\
& \leq c N_{0} \frac{p_{N}}{P_{N}}+\frac{c}{N_{0}+1} \sum_{n=N_{0}}^{N+N_{0}} \frac{1}{P_{N}} \sum_{k=1}^{N_{0}} \underbrace{\left|\tilde{p}_{n-k}-\tilde{p}_{n}\right|}_{\leq \sum_{r=n-k}^{n-1}\left|\tilde{p}_{r}-\tilde{p}_{r+1}\right|} \\
& \leq \epsilon / 4+\frac{c}{N_{0}+1} \sum_{n=0}^{N} \frac{1}{P_{N}}\left|p_{n}-\tilde{p}_{n+1}\right| \sum_{k=1}^{N_{0}} k \\
& \leq \epsilon / 4+\frac{c}{N_{0}+1} \frac{N_{0}\left(N_{0}+1\right)}{2} \frac{1}{P_{N}}\left(2 p_{N}-p_{0}\right)<\epsilon / 2
\end{aligned}
$$

by (12). Hence, $\left\|\frac{1}{P_{N}} \sum_{n=0}^{N} p_{n} x_{n}\right\| \leq \epsilon / 4+\epsilon / 4+\epsilon / 2=\epsilon$.

\section{Remark:}

As in Theorem 3.5, it is easy to see that the same conclusion is true if $p_{n}$ itself is not increasing but there exist some increasing sequence $q_{n}$ and a bounded sequence $a_{n}$, with $p_{n}=q_{n}+a_{n}$. We will call such a sequence almost increasing.

\section{Convergence theorem for functions from $\mathcal{F} \cup \mathcal{L}$}

Theorem 4.3 below will be used as a base of induction in the proofs of Theorems A and B. We will use the following version of van der Corput's difference theorem which follows from the proof of [2, Theorem 1.5].

Theorem 4.1 (Van der Corput trick) Suppose that $x_{n}, n=1,2, \ldots$, is a bounded sequence of elements in a real Hilbert space. If

$$
\lim _{H \rightarrow \infty} \frac{1}{H} \sum_{h=1}^{H} \limsup _{N \rightarrow \infty} \frac{1}{N} \sum_{n=1}^{N}\left\langle x_{n}, x_{n+h}\right\rangle=0,
$$

then

$$
\lim _{N \rightarrow \infty}\left\|\frac{1}{N} \sum_{n=1}^{N} x_{n}\right\|=0
$$

Lemma 4.2 Suppose that $(X, \mathcal{B}, \mu, T)$ is a weakly mixing system and let $q_{i}(x)=\left[\alpha_{i} x\right]+c_{i}$, $\alpha_{i} \in \mathbf{R} \backslash\{0\}, c_{i} \in \mathbf{Z}, i=1, \ldots, k$. Then for any $f_{1}, f_{2}, \ldots, f_{k} \in L^{\infty}(X, \mathcal{B}, \mu)$ one has:

$$
\lim _{N \rightarrow \infty} \frac{1}{N} \sum_{n=1}^{N}\left|\int f_{1} T^{q_{1}(n)} f_{1} d \mu \int f_{2} T^{q_{2}(n)} f_{2} d \mu \cdots \int f_{k} T^{q_{k}(n)} f_{k} d \mu-\prod_{i=1}^{k}\left(\int f_{i} d \mu\right)^{2}\right|=0
$$


Proof: Since $\left\{\left[\alpha_{i} n\right]+c_{i} \mid n \in \mathbf{N}\right\}$, has positive density, we have by Theorem 3.1 that

$$
\lim _{N \rightarrow \infty} \frac{1}{N} \sum_{n=1}^{N}\left|\int f_{i} T^{\left[\alpha_{i} n\right]+c_{i}} f_{i} d \mu-\left(\int f_{i} d \mu\right)^{2}\right|=0, i=1, \ldots, k .
$$

Formula (13) now follows from the fact that if $u_{1}, \ldots, u_{k}$ are bounded sequences and $l_{1}, \ldots, l_{k} \in$ $\mathbf{R}$ with $\lim _{N \rightarrow \infty} \frac{1}{N} \sum_{n=1}^{N}\left|u_{i}(n)-l_{i}\right|=0, i=1, \ldots, k$, then $\lim _{N \rightarrow \infty} \frac{1}{N} \sum_{n=1}^{N}\left|\prod_{i} u_{i}(n)-\prod_{i} l_{i}\right|=$ 0 .

Theorem 4.3 Let $g_{1}, \ldots, g_{k} \in \mathcal{F} \cup \mathcal{L}$ be such that $g_{i}-g_{j} \in \mathcal{F} \cup \mathcal{L}$ for $i \neq j$ and assume that $\left\{g_{1}, \ldots, g_{k}\right\}$ has the $M$-property. Then for any invertible weakly mixing system $(X, \mathcal{B}, \mu, T)$ and any $f_{1}, \ldots, f_{k} \in L^{\infty}(X, \mathcal{B}, \mu)$,

$$
\lim _{N \rightarrow \infty}\left\|\frac{1}{N} \sum_{n=1}^{N} T^{\left[g_{1}(n)\right]} f_{1} \cdots T^{\left[g_{k}(n)\right]} f_{k}-\prod_{i=1}^{k} \int f_{i}\right\|_{2}=0 .
$$

Proof: We use induction on $k$. Let $k=1$. We have to show that for $g \in \mathcal{F} \cup \mathcal{L}$,

$$
\lim _{N \rightarrow \infty}\left\|\frac{1}{N} \sum_{n=1}^{N} T^{[g(n)]} f-\int f\right\|_{2}=0 .
$$

If $g^{\prime}(x) \rightarrow \gamma \neq 0$ as $x \rightarrow \infty$ then $\{[g(n)] \mid n \in \mathbf{N}\}$ has positive lower density and (15) follows from Theorem 3.1 (iv).

If $g^{\prime}(n) \rightarrow 0$ then (15) can be proved in two different ways. One of the proofs works for any Fejér function and is a special case of the proof of Theorem 7.1 below. The other proof, which we will present here, is via the method of passing to an equivalent method of summation and uses the assumption that for some $0<\alpha \leq 1, \lim _{x \rightarrow \infty} \frac{x g^{\prime}(x)}{g(x)}=\alpha$. We remark that this method will be utilized later in the proof of the inductive step.

Replacing, if needed, $f$ by $f-\int f$, we may and will assume that $\int f=0$.

If $g$ is positive, let $\phi(n)=|\{m \in \mathbf{N} \mid[g(m)]=n\}|$. By Lemma 2.4, Lemma 2.5 and Theorem 3.5, we have

$$
\begin{aligned}
\lim _{N \rightarrow \infty} \frac{1}{N} \sum_{n=1}^{N} T^{[g(n)]} f & =\lim _{N \rightarrow \infty} \frac{1}{N} \sum_{n=0}^{[g(N)]} \phi(n) T^{n} f \\
& =\lim _{N \rightarrow \infty} \frac{1}{\sum_{n=0}^{N} \phi(n)} \sum_{n=0}^{N} \phi(n) T^{n} f=\lim _{N \rightarrow \infty} \frac{1}{N} \sum_{n=1}^{N} T^{n} f=\int f .
\end{aligned}
$$

Now if $g$ takes negative values, then $[g(n)]=-[-g(n)]-a_{n}, a_{n} \in\{0,1\}$ and we let $\phi(m)=$ $|\{n \mid[-g(n)]=m\}|$ so that $T^{[g(n)]}=T^{-m-a_{n}} f$. Since $T$ is invertible and weakly mixing we get the limit (15) in the same way as for positive $g$ by using Proposition 3.3.

Suppose that the theorem is true for $k-1$, and let $g_{1}, g_{2}, \ldots, g_{k} \in \mathcal{F} \cup \mathcal{L}$ such that they satisfy the conditions of the theorem. Let $(X, \mathcal{B}, \mu, T)$ be a weakly mixing system and $f_{1}, \ldots, f_{k} \in$ $L^{\infty}(X, \mathcal{B}, \mu)$. We will show (14) for this system. We may assume that $\int f_{1}=0$. 
By the M-property and l'Hopital's rule, $\lim _{n \rightarrow \infty} \frac{g_{i}(n)}{g_{j}(n)}$ exists in $\mathbf{R} \cup\{ \pm \infty\}$ for any $i, j$. By possibly reordering the $g_{i}$ 's, we have that $\lim _{n \rightarrow \infty} \frac{g_{i}(n)}{g_{1}(n)}=\gamma_{i}$, where $\left|\gamma_{i}\right|<\infty$ for all $i$. Consider first the case where $g_{i}^{\prime}(n) \rightarrow \lambda_{i} \neq 0, i=1, \ldots, r$ and $g_{i}^{\prime}(n) \rightarrow 0, i=r+1, \ldots, k$, where $1 \leq r \leq k$. Let $x_{n}=T^{\left[g_{1}(n)\right]} f_{1} \cdots T^{\left[g_{k}(n)\right]} f_{k}$. Then

$$
\begin{aligned}
\left\langle x_{n}, x_{n+h}\right\rangle= & \int T^{\left[g_{1}(n)\right]} f_{1} \cdots T^{\left[g_{k}(n)\right]} f_{k} T^{\left[g_{1}(n+h)\right]} f_{1} \cdots T^{\left[g_{k}(n+h)\right]} f_{k} d \mu \\
= & \int T^{\left[g_{1}(n)\right]}\left(f_{1} T^{\left[g_{1}(n+h)\right]-\left[g_{1}(n)\right]} f_{1}\right) \cdots T^{\left[g_{k}(n)\right]}\left(f_{k} T^{\left[g_{k}(n+h)\right]-\left[g_{k}(n)\right]} f_{k}\right) d \mu \\
= & \int \tilde{f}_{1} T^{\left[g_{2}(n)-g_{1}(n)\right]+c_{2}} \tilde{f}_{2} \cdots T^{\left[g_{r}(n)-g_{1}(n)\right]+c_{r}} \tilde{f}_{r} \\
& \times T^{\left[g_{r+1}(n)-g_{1}(n)\right]+c_{r+1}} \check{f}_{r+1} \cdots T^{\left[g_{k}(n)-g_{1}(n)\right]+c_{k}} \check{f}_{k} d \mu
\end{aligned}
$$

where $\tilde{f}_{i}=f_{i} T^{\left[g_{i}(n+h)\right]-\left[g_{i}(n)\right]} f_{i}=f_{i} T^{\left[\lambda_{i} h\right]+a_{i}(n, h)} f_{i}, i=1, \ldots, r$, and $\check{f}_{i}=f_{i} T^{\left[g_{i}(n+h)\right]-\left[g_{i}(n)\right]} f_{i}=$ $f_{i} T^{a_{i}(n, h)} f_{i}, i=r+1, \ldots, k$, and by Lemma $2.12, c_{i}=c_{i}(n) \in\{0,1\}$ and $a_{i}(n, h) \in\{0, \pm 1\}$ for sufficiently large $n$. For each $h$ and $j=1, \ldots, k$, let $a_{j i}^{h}, c_{j i} \in \mathbf{R}, i=1, \ldots, l$, be such that

$$
\left(a_{1}(n, h), \ldots, a_{k}(n, h), c_{2}(n), \ldots, c_{k}(n)\right) \in\left\{\left(a_{1 i}^{h}, \ldots, a_{k i}^{h}, c_{2 i}, \ldots, c_{k i}\right) \mid i=1, \ldots, l\right\} .
$$

Let $\tilde{f}_{j i}=f_{j} T^{\left[\lambda_{j} h\right]+a_{j i}^{h}} f_{j}, j=1, \ldots, r, \check{f}_{j i}=f_{j} T^{a_{j i}^{h}} f_{j}, j=r+1, \ldots, k$, and define

$$
y_{i}(n, h)=\left|\int \tilde{f}_{1 i} T^{\left[g_{2}(n)-g_{1}(n)\right]+c_{2 i}} \tilde{f}_{2 i} \cdots T^{\left[g_{k}(n)-g_{1}(n)\right]+c_{k i}} \check{f}_{k i} d \mu\right|
$$

for each $n, h, i=1, \ldots, l$. Now, $g_{j}-g_{1} \in \mathcal{F} \cup \mathcal{L}, j=2, \ldots, k$, and these new functions satisfy the conditions of the theorem. Since strong convergence implies weak convergence, and since $T$ is weakly mixing, we have, by the induction hypothesis,

$$
y_{i}(h)=\lim _{N \rightarrow \infty} \frac{1}{N} \sum_{n=1}^{N} y_{i}(n, h)=\left|\int f_{1} T^{\left[\lambda_{1} h\right]+a_{1 i}^{h}} f_{1} \cdots \int f_{r} T^{\left[\lambda_{r} h\right]+a_{r i}^{h}} f_{r} \int \check{f}_{r+1, i} \cdots \int \check{f}_{k i}\right| .
$$

Let $b_{s i j} \in \mathbf{R}$ be such that $\left(a_{1 i}^{h}, \ldots, a_{k i}^{h}\right) \in\left\{\left(b_{1 i j}, \ldots, b_{k i j}\right) \mid j=1, \ldots, u_{i}\right\}$. Let $\check{f}_{s i j}=f_{s} T^{b_{s i j}} f_{s}$, $s=r+1, \ldots, k$, and define

$$
z_{i j}(h)=\left|\int f_{1} T^{\left[\lambda_{1} h\right]+b_{1 i j}} f_{1} \cdots \int f_{r} T^{\left[\lambda_{r} h\right]+b_{r i j}} f_{r} \int \check{f}_{r+1, i j} \cdots \int \check{f}_{k i j}\right|
$$

for each $h$. It follows now from Lemma 4.2 that

$$
\lim _{H \rightarrow \infty} \frac{1}{H} \sum_{h=1}^{H} z_{i j}(h)=\left(\int f_{1}\right)^{2} \cdots\left(\int f_{r}\right)^{2} \int \check{f}_{r+1, i j} \cdots \int \check{f}_{k i j}=0
$$

for each $i, j$ since $\int f_{1}=0$, so that

$$
\lim _{H \rightarrow \infty} \frac{1}{H} \sum_{h=1}^{H} \lim _{N \rightarrow \infty} \frac{1}{N} \sum_{n=1}^{N} y_{i}(n, h)=\lim _{H \rightarrow \infty} \frac{1}{H} \sum_{h=1}^{H} y_{i}(h)=0 .
$$


It follows from Lemma 3.4 that $\lim _{H \rightarrow \infty} \frac{1}{H} \sum_{h=1}^{H} \limsup _{N \rightarrow \infty} \frac{1}{N} \sum_{n=1}^{N}\left|\left\langle x_{n}, x_{n+h}\right\rangle\right|=0$ and so by Theorem 4.1 we have proved (14) for the case where $g_{i}^{\prime}(n) \rightarrow \lambda_{i} \neq 0$ for some $i$.

Suppose now that $g_{i}^{\prime}(n) \rightarrow 0$ for all $i$ and that $\lim _{x \rightarrow \infty} \frac{g_{i}(x)}{g_{1}(x)}=\lim _{x \rightarrow \infty} \frac{g_{i}^{\prime}(x)}{g_{1}^{\prime}(x)}=\gamma_{i}, \gamma_{1}, \ldots, \gamma_{s} \neq 0$, $\gamma_{s+1}=\cdots=\gamma_{k}=0,1 \leq s \leq k$. Introduce the new variable $m, m=\left[g_{1}(n)\right]$ (or $m=\left[-g_{1}(n)\right]$ if $\left.g_{1}<0\right)$. Let $\hat{g}_{i}(m)=g_{i}\left(g_{1}^{-1}(m)\right)$. Then by Lemma 2.7, $\hat{g}_{i} \in \mathcal{F} \cup \mathcal{L}$ and $\left[g_{i}(n)\right]=\left[\hat{g}_{i}\left(\left[g_{1}(n)\right]\right)\right]+$ $c_{i}(n), c_{i}(n) \in C \subset \mathbf{Z},|C|<\infty$, and by Proposition 2.11, $\left\{\hat{g}_{1}, \hat{g}_{2}, \ldots, \hat{g}_{k}\right\}$ has the M-property. Let $\bigcup_{j=1}^{l} C_{j}$ be a partition of $\mathbf{N}$ and $b_{i j} \in \mathbf{R}$ such that $c_{i}(n)=b_{i j}$ if $n \in C_{j}, i=1, \ldots, k$. For $n \in C_{j}$, we then have

$$
T^{\left[g_{1}(n)\right]} f_{1} \cdots T^{\left[g_{k}(n)\right]} f_{k}=T^{\left[g_{1}(n)\right]} f_{1} T^{\left[\hat{g}_{2}\left(\left[g_{1}(n)\right]\right)\right]+b_{2 j} j} f_{2} \cdots T^{\left[\hat{g}_{k}\left(\left[g_{k}(n)\right]\right)\right]+b_{k j}} f_{k},
$$

and by Proposition 3.3 it suffices to prove that

$$
\frac{1}{N} \sum_{n=1}^{N} T^{\left[g_{1}(n)\right]} f_{1} T^{\left[\hat{g}_{2}\left(\left[g_{1}(n)\right]\right)\right]+b_{2 j}} f_{2} \cdots T^{\left[\hat{g}_{k}\left(\left[g_{k}(n)\right]\right)\right]+b_{k j}} f_{k} \rightarrow \int f_{1} \cdots \int f_{k}=0
$$

for $j=1, \ldots, l$. By changing the index of summation, $m=\left[g_{1}(n)\right]$, and letting $\phi(m)=\mid\{n \in$ $\left.\mathbf{N} \mid\left[g_{1}(n)\right]=m\right\} \mid$, we have by Lemmas 2.4, 2.5 and Theorem 3.5

$$
\begin{aligned}
& \lim _{N \rightarrow \infty} \frac{1}{N} \sum_{n=1}^{N} T^{\left[g_{1}(n)\right]} f_{1} T^{\left[\hat{g}_{2}\left(\left[g_{1}(n)\right]\right)\right]+b_{2 j}} f_{2} \cdots T^{\left[\hat{g}_{k}\left(\left[g_{k}(n)\right]\right)\right]+b_{k j}} f_{k} \\
& =\lim _{N \rightarrow \infty} \frac{1}{N} \sum_{m=1}^{\left[g_{1}(N)\right]} \phi(m) T^{m} f_{1} T^{\left[\hat{g}_{2}(m)\right]+b_{2 j}} f_{2} \cdots T^{\left[\hat{g}_{k}(m)\right]+b_{k j}} f_{k} \\
& =\lim _{N \rightarrow \infty} \frac{1}{N} \sum_{m=1}^{N} T^{m} f_{1} T^{\left[\hat{g}_{2}(m)\right]+b_{2 j}} f_{2} \cdots T^{\left[\hat{g}_{k}(m)\right]+b_{k j}} f_{k} .
\end{aligned}
$$

We are now back to our previous situation. Hence, (14) is proved.

Note that in the special case when $g_{1}, \ldots, g_{k} \in \mathcal{L}$, Theorem 4.3 also admits the uniform version which corresponds to replacing the expressions $\lim _{N \rightarrow \infty} \frac{1}{N} \sum_{n=1}^{N} T^{\left[g_{1}(n)\right]} f_{1} \cdots T^{\left[g_{k}(n)\right]} f_{k}$ by $\lim _{N-M \rightarrow \infty} \frac{1}{N-M} \sum_{n=M+1}^{N} T^{\left[g_{1}(n)\right]} f_{1} \cdots T^{\left[g_{k}(n)\right]} f_{k}$. The proof follows by invoking the uniform version of Theorem 4.1 (see Theorem 3.2 in [2]) and is left to the reader. In particular, we have the following result which will be used in the proof of Theorem 5.13.

Proposition 4.4 Let $c_{1}, \ldots, c_{k} \in \mathbf{R} \backslash\{0\}$ and assume that $c_{i} \neq c_{j}$ for $i \neq j$. Then for any invertible weakly mixing system $(X, \mathcal{B}, \mu, T)$ and any $f_{1}, \ldots, f_{k} \in L^{\infty}(X, \mathcal{B}, \mu)$,

$$
\lim _{N \rightarrow \infty}\left\|\frac{1}{N} \sum_{n=m+1}^{m+N} T^{\left[c_{1} n\right]} f_{1} \cdots T^{\left[c_{k} n\right]} f_{k}-\prod_{i=1}^{k} \int f_{i}\right\|_{2}=0 \quad \text { uniformly in } m .
$$




\section{$5 \quad$ Tempered functions}

In this section we take a closer look at the various classes of tempered functions which were defined in the Introduction. The results obtained in this section will be used in the next section in the proofs of Theorems A and B.

Proposition 5.1 Let $\mathcal{G}_{\mathcal{H}}=\left\{g \in \mathcal{H} \mid \exists \alpha>0, \lim _{x \rightarrow \infty} \frac{x g^{\prime}(x)}{g(x)}=\alpha\right.$, if $\alpha \in \mathbf{N}$ then $\lim _{x \rightarrow \infty} g^{(\alpha)}(x) \in$ $\mathbf{R}\}$. Then $\mathcal{G}_{\mathcal{H}}=\mathcal{G} \cap \mathcal{H}$.

Proof: In order to use l'Hopital's rule when the functions involved are members of a Hardy field, we only need to check that both numerator and denominator either tend to 0 or to $\pm \infty$. We leave it to the reader to justify the use of l'Hopital's rule below, but remark that Lemma 2.1 is often useful.

$\mathcal{G} \cap \mathcal{H} \subset \mathcal{G}_{\mathcal{H}}$ : Clearly $\mathcal{T} \cap \mathcal{H} \subset \mathcal{G}_{\mathcal{H}}$. Let $g \in \mathcal{P}_{l} \cap \mathcal{H}$. Then $\lim _{x \rightarrow \infty} \frac{x g^{(l+2)}}{g^{(l+1)}}=\frac{0}{\gamma}=0$. By l'Hopital's rule we have $\lim _{x \rightarrow \infty} \frac{x g^{\prime}}{g}=l+1$. Hence, $g \in \mathcal{G}_{\mathcal{H}}$.

$\mathcal{G}_{\mathcal{H}} \subset \mathcal{G} \cap \mathcal{H}$ : Let $g \in \mathcal{G}_{\mathcal{H}}, l \in \mathbf{N} \cup\{0\}$ and $l<\alpha \leq l+1$ such that $\lim _{x \rightarrow \infty} \frac{x g^{\prime}}{g}=\alpha$. If $\alpha \neq l+1$ or $\alpha=l+1$ and $\lim _{x \rightarrow \infty} g^{(l+1)}(x)=0$ then it follows by l'Hopital's rule that $\lim _{x \rightarrow \infty} \frac{x g^{(i+1)}(x)}{g^{(i)}(x)}=\alpha-i$ for all $i \in \mathbf{N}$, and hence, $g \in \mathcal{T}$. If $\alpha=l+1$ then by l'Hopital's rule $\lim _{x \rightarrow \infty} \frac{x g^{(l+2)}(x)}{g^{(l+1)}(x)}=0$ so that if $\lim _{x \rightarrow \infty} g^{(l+1)}(x)=\gamma \neq 0$, then $\lim _{x \rightarrow \infty} x g^{(l+2)}(x)=0$. In particular, $\lim _{x \rightarrow \infty} g^{(l+2)}(x)=0$. So by l'Hopital's rule again, $0=\lim _{x \rightarrow \infty} x g^{(l+2)}(x)=$ $\lim _{x \rightarrow \infty} \frac{g^{(l+2)}(x)}{1 / x}=-\lim _{x \rightarrow \infty} \frac{g^{(l+3)}(x)}{1 / x^{2}}=-\lim _{x \rightarrow \infty} x^{2} g^{(l+3)}(x)$. By induction, $\lim _{x \rightarrow \infty} x^{i} g^{(l+i+1)}(x)=0$ for all $i \in \mathbf{N}$. Hence, $g \in \mathcal{P}$.

Proposition 5.2 If $g \in \mathcal{T}$ then $g$ is a tempered function.

Proof: $\quad$ Suppose that $l \geq 0, g \in \mathcal{T}_{l}$ and that $0<\lambda \leq 1$ such that $\lim _{x \rightarrow \infty} \frac{x g^{\prime}}{g}=\lambda+l$. Since $\mathcal{T} \subset \mathcal{R}$, all the derivatives $g^{(i)}$ has eventually constant sign. Also, by l'Hopital's rule, $\lim _{x \rightarrow \infty} \frac{x g^{(l+1)}}{g^{(l)}}=\lambda$. Hence, by Lemma 2.2, $g^{(l)}$ is a Fejér function and therefore $g$ is a tempered function of order $l$.

Definition 5.3 Let

$$
\mathcal{S}=\left\{f \in C^{\infty}\left(\mathbf{R}^{+}\right) \mid \lim _{x \rightarrow \infty} \frac{x^{i} f^{(i)}(x)}{f(x)}=0 \text { for all } i \in \mathbf{N}\right\}
$$

Examples: The following functions belong to $\mathcal{S}$ :

- $\gamma+\sum_{i=1}^{k} c_{i} x^{\beta_{i}}$, where $\gamma \neq 0, c_{i} \in \mathbf{R}, \beta_{i}<0, i=1, \ldots, k$.

- $\gamma+\cos \left(\log ^{\beta} x\right)$, where $|\gamma|>1,0<\beta<1$ or $\gamma \in \mathbf{R}$ and $\beta<0$.

- $\gamma+\log ^{\beta} x$, where $\gamma \in \mathbf{R}, \beta \in \mathbf{R}$ 
- $\gamma+\frac{\sin (\log x)}{\log x}$, where $\gamma \neq 0$.

Proposition 5.4 Let $l \in \mathbf{N} \cup\{0\}$.

(i) Let $0<\lambda<1$. Then $g \in \mathcal{T}_{l}$ with $\lim _{x \rightarrow \infty} \frac{x g^{\prime}(x)}{g^{\prime}(x)}=\lambda+l$ if and only if there exists $f \in \mathcal{S}$ such that $g(x)=x^{\lambda+l} f(x)$.

(ii) If $g \in \mathcal{T}_{l}$ with $\lim _{x \rightarrow \infty} \frac{x g^{\prime}(x)}{g^{\prime}(x)}=l+1$ then there exists $f \in \mathcal{S}$ with $\lim _{x \rightarrow \infty} f(x)=0$ such that $g(x)=x^{l+1} f(x)$.

(iii) Let $\gamma \in \mathbf{R} \backslash\{0\}$. Then $g \in \mathcal{P}_{l}$ with $\lim _{x \rightarrow \infty} g^{(l+1)}(x)=\gamma$ if and only if there exists $f \in \mathcal{S}$ with $\lim _{x \rightarrow \infty} f(x)=\frac{\gamma}{(l+1) !}$ and such that $g=x^{l+1} f$.

Proof: $\quad\left(\right.$ i) $(\Rightarrow)$ and (ii). Let $0<\lambda \leq 1$. Suppose that $g \in \mathcal{T}_{l}$ and that $\lim _{x \rightarrow \infty} \frac{x g^{\prime}(x)}{g(x)}=\lambda+l$. By l'Hopital's rule,

$$
\lim _{x \rightarrow \infty} \frac{x g^{(i+1)}(x)}{g^{(i)}(x)}=\lambda+l-i
$$

for $i=0,1,2, \ldots$ Let $\alpha=\lambda+l$ and let $f(x)=\frac{g(x)}{x^{\alpha}}$. Then

$$
g^{(i)}(x)=\sum_{s=0}^{i} a_{i s} x^{\alpha-s} f^{(i-s)}(x), \quad i=0,1, \ldots
$$

where $a_{i 0}=1$ for all $i$ and $a_{i s}=\left(\begin{array}{c}i \\ s\end{array}\right) \prod_{j=0}^{s-1}(\alpha-j)$ for $s=1, \ldots, i, i \geq 1$. Hence $a_{i s} \neq 0$ for $s=0, \ldots, i$ and for all $i$ if $\lambda<1$ and for $i<l+2$ if $\lambda=1$ so that

$$
\frac{x g^{(i+1)}(x)}{g^{(i)}(x)}=\frac{(\alpha-i)+\sum_{s=0}^{i} b_{i s} \frac{x^{i+1-s} f^{(i+1-s)}(x)}{f(x)}}{1+\sum_{s=0}^{i-1} c_{i s} \frac{x^{i-s} f^{(i-s)}(x)}{f(x)}}
$$

for $i=0,1,2, \ldots$ when $\lambda<1$ and for $i=0,1, \ldots, l+1$ when $\lambda=1$, where $b_{i s}=\frac{a_{i+1, s}}{\alpha(\alpha-1) \cdots(\alpha-i+1)}$, $c_{i s}=\frac{a_{i s}}{\alpha(\alpha-1) \cdots(\alpha-i+1)}$. When $\lambda=1$ and $i \geq l+2$, then

$$
\frac{x g^{(i+1)}(x)}{g^{(i)}(x)}=\frac{\frac{x^{i+1} f^{(i+1)}(x)}{f(x)}+\sum_{s=1}^{l+1} a_{i+1, s} \frac{x^{i+1-s} f^{(i+1-s)}(x)}{f(x)}}{\sum_{s=0}^{l+1} a_{i s} \frac{x^{i-s} f^{(i-s)}(x)}{f(x)}} .
$$

Since $\alpha=\lim _{x \rightarrow \infty} \frac{x g^{\prime}(x)}{g(x)}$, we get from (18), $i=0$, that $\lim _{x \rightarrow \infty} \frac{x f^{\prime}(x)}{f(x)}=0$, and by induction on $i$, using (16) and (18) when $\lambda<1$ and using (16), (18) and (19) when $\lambda=1$, we get $\lim _{x \rightarrow \infty} \frac{x^{i} f^{(i)}(x)}{f(x)}=0$, $i=1,2, \ldots$. Hence, $g(x)=x^{\alpha} f(x)$ where $f \in \mathcal{S}$. It is left to prove that $\lim _{x \rightarrow \infty} f(x)=0$ when $\lambda=1$. But this follows from the facts that $g \in \mathcal{T}_{l}$ so that $\lim _{x \rightarrow \infty} g^{(l+1)}(x)=0$, and that

$$
g^{(l+1)}(x)=f(x)\left((l+1) !+\sum_{s=0}^{l} a_{l+1, s} \frac{x^{l+1-s} f^{(l+1-s)}(x)}{f(x)}\right) .
$$


(i) $(\Leftarrow)$. Suppose now that $0<\lambda<1$ and that $g=x^{\lambda+l} f, f \in \mathcal{S}$. It follows from (18) that $\lim _{x \rightarrow \infty} \frac{x g^{(i+1)}(x)}{g^{(i)}(x)}=\lambda+l-i$ for $i=0,1,2, \ldots$, and hence, $g \in \mathcal{T}_{l}$.

(iii) Let $\gamma \in \mathbf{R} \backslash\{0\}$ and $g \in C^{\infty}\left(\mathbf{R}^{+}\right)$such that $\lim _{x \rightarrow \infty} g^{(l+1)}(x)=\gamma$. Let also $g_{1}=g^{(l)}$ and $f_{1}=g_{1} / x$. By l'Hopital's rule, $\lim _{x \rightarrow \infty} f_{1}(x)=\lim _{x \rightarrow \infty} g_{1}^{\prime}(x)=\gamma$. Furthermore, we have $g_{1}^{(i)}=i f_{1}^{(i-1)}+x f_{1}^{(i)}$ for $i \in \mathbf{N}$. By letting $i=1$ we get that $\lim _{x \rightarrow \infty} x f_{1}^{\prime}(x)=0$. Hence, by induction on $i$, we have

$$
\lim _{x \rightarrow \infty} x^{i} g_{1}^{(i+1)}(x)=0 \Leftrightarrow \lim _{x \rightarrow \infty} x^{i} f_{1}^{(i)}(x)=0, \quad i \in \mathbf{N} .
$$

$(\Rightarrow)$. Let $g, \gamma$ and $f_{1}$ be as above and assume that $g \in \mathcal{P}_{l}$. Let $f_{2}=\frac{g}{x^{l+1}}$. Then, by l'Hopital's rule, $\lim _{x \rightarrow \infty} f_{2}(x)=\lim _{x \rightarrow \infty} \frac{g^{(l+1)}(x)}{(l+1) !}=\frac{\gamma}{(l+1) !}$. It is left to prove that $f_{2} \in \mathcal{S}$. Since $\frac{x g^{(l+1)}}{g^{(l)}}=\frac{g^{(l+1)}}{f_{1}}$ so that $\lim _{x \rightarrow \infty} \frac{x g^{(l+1)}}{g^{(l)}}=1$, we obtain $(16)$ for $\lambda=1$ and $i=0, \ldots, l$, by l'Hopital's rule. We also have (18) for $f=f_{2}, \lambda=1$ and $i=0, \ldots, l+1$. Hence, $\lim _{x \rightarrow \infty} x^{i} f_{2}^{(i)}(x)=0$ for $i=1, \ldots, l+2$. Since $g^{(l)}=(l+1) ! x f_{2}+\sum_{s=0}^{l-1} a_{l s} x^{l+1-s} f_{2}^{(l-s)}$ (see $(17))$

$$
f_{1}=(l+1) ! f_{2}+\sum_{s=0}^{l-1} a_{l s} x^{l-s} f_{2}^{(l-s)}
$$

so that for some $b_{s j} \in \mathbf{R}, x^{i} f_{1}^{(i)}=(l+1) ! x^{i} f_{2}^{(i)}+\sum_{s=0}^{l-1} a_{l s}\left(\sum_{j=0}^{i} b_{s j} x^{l-s+i-j} f_{2}^{(l-s+i-j)}\right)$. Since $\lim _{x \rightarrow \infty} x^{i} f_{1}^{(i)}(x)=0$ by (21), it follows by inducton on $i$ that $\lim _{x \rightarrow \infty} x^{i} f_{2}^{(i)}(x)=0$ for all $i$. Hence, $f_{2} \in \mathcal{S}$.

$(\Leftarrow)$. Suppose that $g=x^{l+1} f_{2}, f_{2} \in \mathcal{S}, \lim _{x \rightarrow \infty} f_{2}(x)=\gamma_{2} \in \mathbf{R} \backslash\{0\}$. In the same way as we obtained (22) we now get that $g^{(l)}=x f_{1}$ where $f_{1}$ is given by (22). It follows that $\lim _{x \rightarrow \infty} f_{1}(x)=(l+1) ! \gamma_{2}$ and that $f_{1} \in \mathcal{S}$. Hence, $g \in \mathcal{P}_{l}$ by $(21)$.

Lemma 5.5 If $f_{1}, f_{2} \in \mathcal{S}$ and $c \in \mathbf{R} \backslash\{0\}$ then

(i) $f_{1} f_{2} \in \mathcal{S}$

(ii) $1 / f_{1} \in \mathcal{S}$

(iii) $c f_{1}+x f_{1}^{\prime} \in \mathcal{S}$

(iv) $f_{1}+x^{\alpha} f_{2} \in \mathcal{S}$ if $\alpha<0$

(v) $f_{1}+f_{2} \in \mathcal{S}$ if $L=\lim _{x \rightarrow \infty} \frac{f_{1}(x)}{f_{2}(x)}$ exists in $\mathbf{R} \cup\{ \pm \infty\}$ and $L \neq-1$

Proof: $\quad$ (i) Let $f=f_{1} f_{2}$. Since $f^{(i)}=\sum_{s=0}^{i}\left(\begin{array}{c}i \\ s\end{array}\right) f_{1}^{(i-s)} f_{2}^{(s)}$, we have

$$
\lim _{x \rightarrow \infty} \frac{x^{i} f^{(i)}(x)}{f(x)}=\lim _{x \rightarrow \infty} \sum_{s=0}^{i}\left(\begin{array}{c}
i \\
s
\end{array}\right) \frac{x^{i-s} f_{1}^{(i-s)}(x)}{f_{1}(x)} \cdot \frac{x^{s} f_{2}^{(s)}(x)}{f_{2}(x)}=0
$$


for $i \in \mathbf{N}$.

(ii) Let $f=1 / f_{1}$. We show by induction on $i$ that $\frac{x^{i} f^{(i)}}{f}$ is a linear combination of terms of form $\prod_{\sum j=i} \frac{x^{j} f_{1}^{(j)}}{f_{1}}$. When $i=1$ then $\frac{x f^{\prime}}{f}=-\frac{x f_{1}^{\prime}}{f_{1}}$ which is of the right form. Suppose $\frac{x^{i} f^{(i)}}{f}=\sum_{l} c_{l} \phi_{l}, \phi_{l}=\prod_{\sum_{j} l_{j}=i} \frac{x^{l_{j}} f_{1}^{\left(l_{j}\right)}}{f_{1}}$ so that $f^{(i)}=\sum_{l} c_{l} f \prod_{\sum_{j} l_{j}=i} \frac{f_{1}^{\left(l_{j}\right)}}{f_{1}}$ and $f^{\prime}=-f \frac{f_{1}^{\prime}}{f_{1}}$. Then $f^{(i+1)}=\sum_{l} c_{l}\left(f^{\prime} \prod_{\sum_{j} l_{j}=i} \frac{f_{1}^{\left(l_{j}\right)}}{f_{1}}+f \sum_{s} \frac{f_{1} f_{1}^{\left(l_{s}+1\right)}-f_{1}^{\prime} f_{1}^{\left(l_{s}\right)}}{f_{1}^{2}} \prod_{l_{j} \neq l_{s}} \frac{f_{1}^{\left(l_{j}\right)}}{f_{1}}\right)$ so that $\frac{x^{i+1} f^{(i+1)}}{f}=\sum_{l} c_{l}\left(-\frac{x f_{1}^{\prime}}{f_{1}} \prod_{\sum_{j} l_{j}=i} \frac{x^{l_{j}} f_{1}^{\left(l_{j}\right)}}{f_{1}}+\sum_{s}\left(\frac{x^{l_{s}+1} f_{1}^{\left(l_{s}+1\right)}}{f_{1}}-\frac{x f_{1}^{\prime}}{f_{1}} \frac{x^{l_{s}} f_{1}^{\left(l_{s}\right)}}{f_{1}}\right) \prod_{l_{j} \neq l_{s}} \frac{x^{l_{j}} f_{1}^{\left(l_{j}\right)}}{f_{1}}\right)$ which is of the right form. Hence, $\lim _{x \rightarrow \infty} \frac{x^{i} f^{(i)}(x)}{f(x)}=0$ for $i \in \mathbf{N}$ so that $f \in \mathcal{S}$.

(iii) Let $f=c f_{1}+x f_{1}^{\prime}$. Then $f^{(i)}=(c+i) f_{1}^{(i)}+x f_{1}^{(i+1)}$ so that for $i \in \mathbf{N}$,

$$
\lim _{x \rightarrow \infty} \frac{x^{i} f^{(i)}(x)}{f(x)}=\lim _{x \rightarrow \infty} \frac{(c+i) \frac{x^{i} f_{1}^{(i)}(x)}{f_{1}(x)}+\frac{x^{i+1} f_{1}^{(i+1)}(x)}{f_{1}(x)}}{c+\frac{x f_{1}^{\prime}(x)}{f_{1}(x)}}=0 .
$$

(iv) Let $f=f_{1}+x^{\alpha} f_{2}$. It follows by induction on $i$ that $f^{(i)}=f_{1}^{(i)}+x^{\alpha-i}(\alpha(\alpha-1) \cdots(\alpha-i+$ 1) $f_{2}+\sum_{j=1}^{i} c_{j} x^{j} f_{2}^{(j)}$ ) for some $c_{j} \in \mathbf{R}$ so that

$$
\lim _{x \rightarrow \infty} \frac{x^{i} f^{(i)}(x)}{f(x)}=\lim _{x \rightarrow \infty} \frac{\frac{x^{i} f_{1}^{(i)}(x)}{f_{1}(x)}+x^{\alpha} \frac{f_{2}(x)}{f_{1}(x)}\left(\alpha \cdots(\alpha-i+1)+\sum_{j=1}^{i} c_{j} \frac{x^{j} f_{2}^{(j)}(x)}{f_{2}(x)}\right)}{1+x^{\alpha} \frac{f_{2}(x)}{f_{1}(x)}}=0
$$

for $i \in \mathbf{N}$, since by (i) and (ii), $\frac{f_{2}}{f_{1}} \in \mathcal{S}$ and $\lim _{x \rightarrow \infty} x^{\alpha} \frac{f_{2}(x)}{f_{1}(x)}=0$ by Lemma 2.1 .

(v) Let $f=f_{1}+f_{2}$. Suppose that $\lim _{x \rightarrow \infty}\left|\frac{f_{2}}{f_{1}}\right|<\infty$. Then for all $i \in \mathbf{N}$,

$$
\lim _{x \rightarrow \infty} \frac{x^{i} f^{(i)}(x)}{f(x)}=\lim _{x \rightarrow \infty} \frac{\frac{x^{i} f_{1}^{(i)}(x)}{f_{1}(x)}+\frac{f_{2}(x)}{f_{1}(x)} \frac{x^{i} f_{2}^{(i)}(x)}{f_{2}(x)}}{1+\frac{f_{2}(x)}{f_{1}(x)}}=0 .
$$

Remark: If $c=0$ then Lemma 5.5 (iii) is true only for some $f_{1} \in \mathcal{S}$. For example, if $f_{1}(x)=\log x$ then $x f_{1}^{\prime}=1$ which is in $\mathcal{S}$, while if $f_{1}(x)=1+\frac{1}{x}$ then $x f_{1}^{\prime}(x)=-\frac{1}{x} \notin \mathcal{S}$. Note also that Lemma 5.5 (iv) is not true for $\alpha>0$. For $f_{1}+x^{\alpha} f_{2}=x^{\alpha}\left(f_{2}+x^{-\alpha} f_{1}\right)$ so that if $x^{\alpha} f_{2} \in \mathcal{G}$, then also $f_{1}+x^{\alpha} f_{2} \in \mathcal{G}$ and therefore $f_{1}+x^{\alpha} f_{2} \notin \mathcal{S}$. 
Lemma 5.6 Let $0<\alpha_{1}<\alpha_{2}$ and suppose that

$$
g_{1} \in \mathcal{G}\left(\alpha_{1}\right), \quad g_{2} \in \mathcal{G}\left(\alpha_{2}\right), \quad g(x)=g_{1}(x)+g_{2}(x) .
$$

Then $g \in \mathcal{G}\left(\alpha_{2}\right)$ and $g \in \mathcal{P}$ if and only if $g_{2} \in \mathcal{P}$.

Proof: By Proposition 5.4, there exist $f_{1}, f_{2} \in \mathcal{S}$ such that $g_{1}=x^{\alpha_{1}} f_{1}$ and $g_{2}=x^{\alpha_{2}} f_{2}$. We have $g=g_{1}+g_{2}=x^{\alpha_{2}}\left(x^{\alpha_{1}-\alpha_{2}} f_{1}+f_{2}\right)$ and $f=x^{\alpha_{1}-\alpha_{2}} f_{1}+f_{2} \in \mathcal{S}$ by Lemma 5.5(iv). Hence, if $\alpha_{2} \notin \mathbf{N}$, then $g \in \mathcal{T}\left(\alpha_{2}\right)$ by Proposition 5.4.

If $\alpha_{2}=l \in \mathbf{N}$ and $\lim _{x \rightarrow \infty} f_{2}(x)=\gamma$, then $\lim _{x \rightarrow \infty} f(x)=\lim _{x \rightarrow \infty}\left(x^{\alpha_{1}-l} f_{1}(x)+f_{2}(x)\right)=\gamma$. Hence, $g \in \mathcal{P}_{l-1}$ if $\gamma \neq 0$ by Proposition 5.4(iii).

Let $\alpha_{2}=l \in \mathbf{N}$ and $\gamma=0$. If $g_{1}, g_{2} \in \mathcal{T}$ then it follows from Lemma 2.6 that $\lim _{x \rightarrow \infty} \frac{g_{1}^{(i)}(x)}{g_{2}^{(i)}(x)}=0$ for all $i \in \mathbf{N}$ such that

$$
\lim _{x \rightarrow \infty} \frac{x g^{(i+1)}(x)}{g^{(i)}(x)}=\lim _{x \rightarrow \infty} \frac{\frac{x g_{2}^{(i+1)}(x)}{g_{2}^{(i)}(x)}+\frac{x g_{1}^{(i+1)}(x)}{g_{1}^{(i)}(x)} \frac{g_{1}^{(i)}(x)}{g_{2}^{(i)}(x)}}{1+\frac{g_{1}^{(i)}(x)}{g_{2}^{(i)}(x)}}=\lim _{x \rightarrow \infty} \frac{x g_{2}^{(i+1)}(x)}{g_{2}^{(i)}(x)}=\alpha_{2}-i
$$

which shows that $g \in \mathcal{T}\left(\alpha_{2}\right)$. Suppose now that $g_{1} \in \mathcal{P}$ and $g_{2} \in \mathcal{T}$. We have $g=g_{2}(1+$ $\left.\frac{g_{1}}{g_{2}}\right)=g_{2}\left(1+x^{\alpha_{1}-l} \frac{f_{1}}{f_{2}}\right)$. Let $\phi=1+x^{\alpha_{1}-l} \frac{f_{1}}{f_{2}}$. By Lemma $5.5, \phi \in \mathcal{S}$. For any $j \in \mathbf{N}$, $g^{(j)}=\sum_{s=0}^{j}\left(\begin{array}{l}j \\ s\end{array}\right) g_{2}^{(j-s)} \phi^{(s)}$ and for $i \in \mathbf{N}$

$$
\frac{x g^{(i+1)}(x)}{g^{(i)}(x)}=\frac{x \sum_{s=0}^{i+1}\left(\begin{array}{c}
i+1 \\
s
\end{array}\right) g_{2}^{(i+1-s)} \phi^{(s)}}{\sum_{s=0}^{i}\left(\begin{array}{c}
i \\
s
\end{array}\right) g_{2}^{(i-s)} \phi^{(s)}}=\frac{\frac{x g_{2}^{(i+1)}(x)}{g_{2}^{(i)}(x)}+\sum_{s=1}^{i+1}\left(\begin{array}{c}
i+1 \\
s
\end{array}\right) \frac{g_{2}^{(i+1-s)}(x)}{g_{2}^{(i)}(x)} \frac{x \phi^{(s)}(x)}{\phi(x)}}{1+\sum_{s=1}^{i}\left(\begin{array}{c}
i \\
s
\end{array}\right) \frac{g_{2}^{(i-s)}}{g_{2}^{(i)}} \frac{\phi^{(s)}}{\phi}} .
$$

Since $\phi \in \mathcal{S}$ such that $\frac{x^{s} \phi^{(s)}}{\phi} \rightarrow 0, \frac{g_{2}^{(i+1-s)}}{g_{2}^{(i)}} \frac{x \phi^{(s)}}{\phi}=\frac{g_{2}^{(i-1)}}{x g_{2}^{(i)}} \frac{g_{2}^{(i-2)}}{x g_{2}^{(i-1)}} \cdots \frac{g_{2}^{(i+1-s)}}{x g_{2}^{(i+2-s)}} \frac{x^{s} \phi^{(s)}}{\phi}$ goes to 0 if $l \notin\{i-1, \ldots, i+1-s\}$. Suppose that $l \in\{i-1, \ldots, i+1-s\}$, and let $\tilde{f}=\frac{g_{2}^{(l+1)}}{x g_{2}^{(l+2)}}$. Then $\lim _{x \rightarrow \infty} \frac{x \tilde{f}^{\prime}}{\tilde{f}}=0$. We will show that $\lim _{x \rightarrow \infty} \tilde{f} x^{s} \phi^{(s)}=0$ for $s \in \mathbf{N}$. Let $\lambda=\alpha_{1}-l$ and $\psi=f_{1} / f_{2} \in \mathcal{S}$ such that $\phi=1+x^{\lambda} \psi$. Then

$$
\phi^{(s)}=x^{\lambda-s} \psi\left(\prod_{j=0}^{s-1}(\lambda-j)+\sum_{j=0}^{i-1} a_{s j} \frac{x^{s-j} \psi^{(s-j)}}{\psi}\right),
$$

where $a_{s j} \in \mathbf{R}$. Hence, $\lim _{x \rightarrow \infty} \tilde{f} x^{s} \phi^{(s)}=0$ if $\lim _{x \rightarrow \infty} x^{\lambda} \psi \tilde{f}=0$. Let $\hat{g}=x^{\lambda} \psi \tilde{f}$. Then $\lim _{x \rightarrow \infty} \frac{x \hat{g}^{\prime}}{\hat{g}}=\lambda<0$. Hence, by Lemma $2.1, \lim _{x \rightarrow \infty} \tilde{f} x^{s} \phi^{(s)}=0$. It follows that

$\lim _{x \rightarrow \infty} \frac{g_{2}^{(i+1-s)}(x)}{g_{2}^{(i)}(x)} \frac{x \phi^{(s)}(x)}{\phi(x)}=0$ for all $s \in \mathbf{N}$. In the same way, $\lim _{x \rightarrow \infty} \frac{g_{2}^{(i-s)}(x)}{g_{2}^{(i)}(x)} \frac{\phi^{(s)}(x)}{\phi(x)}=0$ for all $s \in \mathbf{N}$. Hence, by $(23), \lim _{x \rightarrow \infty} \frac{x g^{(i+1)}(x)}{g^{(i)}(x)}=\lim _{x \rightarrow \infty} \frac{x g_{2}^{(i+1)}(x)}{g_{2}^{(i)}(x)}$ for all $i \in \mathbf{N}$, and therefore 
$g \in \mathcal{T}$.

If $g \in \mathcal{G}$ and $h \in \mathbf{N}$, we will use the notation

$$
\Delta_{h} g(x)=g(x+h)-g(x) .
$$

It is not hard to show that if $g$ is a tempered function of order $i$ and $\beta>0$ then $g(x+\beta)-g(x)$ is tempered of order $i-1$ (see for example [17, p. 36]). We have the following generalization of this fact.

Lemma 5.7 Let $\alpha>1$ and $g \in \mathcal{G}(\alpha)$. Then $\Delta_{h} g \in \mathcal{G}(\alpha-1)$ and $g \in \mathcal{T}$ if and only if $\Delta g \in \mathcal{T}$.

Proof: If $l>1$ and $g \in \mathcal{P}_{l}$ such that $\lim _{x \rightarrow \infty} g^{(l+1)}(x)=\gamma \neq 0$, and $\lim _{x \rightarrow \infty} x^{i} g^{(l+i+1)}(x)=$ 0 for all $i \in \mathbf{N}$, then by the mean value theorem there exists $z_{i}=z_{i}(x), x<z_{i}<x+$ $h$, such that $\lim _{x \rightarrow \infty}\left(\Delta_{h} g\right)^{(l)}(x)=\lim _{x \rightarrow \infty} g^{(l+1)}\left(z_{0}\right) h=\gamma h$ and $\lim _{x \rightarrow \infty} x^{i}\left(\Delta_{h} g\right)^{(l+i)}(x)=$ $\lim _{x \rightarrow \infty} x^{i} g^{(l+i+1)}\left(z_{i}\right)=0$ for all $i \in \mathbf{N}$. Hence, $\Delta_{h} g \in \mathcal{P}_{l-1}$.

Let $g \in \mathcal{T}$ and $i \geq 1$. Then for some $0 \leq c_{x}, d_{x}<h$ and by Lemma $2.3, \lim _{x \rightarrow \infty} \frac{x\left(\Delta_{h} g\right)^{(i)}(x)}{\left(\Delta_{h} g\right)^{(i-1)}(x)}=$ $\lim _{x \rightarrow \infty} \frac{x g^{(i+1)}\left(x+c_{x}\right) h}{g^{(i)}\left(x+d_{x}\right) h}=\lim _{x \rightarrow \infty} \frac{x g^{(i+1)}(x)}{g^{(i)}(x)}=\alpha-i$. Also, if $\alpha=l \in \mathbf{N}$ then $\lim _{x \rightarrow \infty}\left(\Delta_{h} g\right)^{(l-1)}(x)=$ $h \lim _{x \rightarrow \infty} g^{(l)}(x)=0$. Hence, $\Delta_{h} g \in \mathcal{T}(\alpha-1)$ for all $\alpha>0$.

Lemma 5.8 Let $\alpha>0, l=[\alpha]$ and let $g_{1}, g_{2} \in \mathcal{T}(\alpha)$ be such that either $\lim _{x \rightarrow \infty} \frac{g_{1}^{(l+1)}(x)}{g_{2}^{(l+1)}(x)}$ exists in $\mathbf{R} \cup\{ \pm \infty\}$ or if $\alpha \notin \mathbf{N}, \lim _{x \rightarrow \infty} \frac{g_{1}(x)}{g_{2}(x)}$ exists in $\mathbf{R} \cup\{ \pm \infty\}$. Then $\lim _{x \rightarrow \infty} \frac{g_{1}^{(i)}(x)}{g_{2}^{(i)}(x)}=$ $\lim _{x \rightarrow \infty} \frac{g_{1}(x)}{g_{2}(x)}$ for all $i \in \mathbf{N}$.

Proof: $\quad$ Since if $g \in \mathcal{T}(\alpha)$, then $\lim _{x \rightarrow \infty} \frac{x g^{(i+1)}(x)}{g^{(i)}(x)}=\alpha-i$ for all $i \in \mathbf{N}$, we have that

$$
\lim _{x \rightarrow \infty} \frac{g_{1}^{(i)}(x)}{g_{2}^{(i)}(x)}=\lim _{x \rightarrow \infty} \frac{\frac{x g_{1}^{(i)}(x)}{g_{1}^{(i-1)}(x)}}{\frac{x g_{2}^{(i)}(x)}{g_{2}^{(i-1)}(x)}} \frac{g_{1}^{(i-1)}(x)}{g_{2}^{(i-1)}(x)}=\frac{\alpha-i+1}{\alpha-i+1} \lim _{x \rightarrow \infty} \frac{g_{1}^{(i-1)}(x)}{g_{2}^{(i-1)}(x)}
$$

for all $i \in \mathbf{N}$ for which $\alpha \neq i-1$. If $\alpha \notin \mathbf{N}$ and $\lim _{x \rightarrow \infty} \frac{g_{1}(x)}{g_{2}(x)}$ exists, the conclusion follows by induction on $i$. Suppose that $\alpha \in \mathbf{R}^{+}$and that $\lim _{x \rightarrow \infty} \frac{g_{1}^{(l+1)}(x)}{g_{2}^{(l+1)}(x)}$ exists. Then by induction and (24), $\lim _{x \rightarrow \infty} \frac{g_{1}^{(l+i)}(x)}{g_{2}^{(l+i)}(x)}=\lim _{x \rightarrow \infty} \frac{g_{1}^{(l+1)}(x)}{g_{2}^{(l+1)}(x)}$ for all $i \in \mathbf{N}$. Furthermore, $\lim _{x \rightarrow \infty} \frac{g_{1}^{(i)}(x)}{g_{2}^{(i)}(x)}=$ $\lim _{x \rightarrow \infty} \frac{g_{1}^{(l+1)}(x)}{g_{2}^{(l+1)}(x)}$ for all $0 \leq i \leq l$ by l'Hopital's rule.

Lemma 5.9 Let $0<\alpha_{1} \leq \alpha_{2}, \alpha_{2}>1$. Suppose that $g_{1} \in \mathcal{G}\left(\alpha_{1}\right)$ and $g_{2} \in \mathcal{G}\left(\alpha_{2}\right)$ are such that $g_{2}-g_{1} \in \mathcal{G}$ and such that if $\left[\alpha_{2}\right]=l$ and $g_{2}-g_{1} \in \mathcal{T}\left(\alpha_{2}-1\right), g_{2} \in \mathcal{T}\left(\alpha_{2}\right)$, then $\lim _{x \rightarrow \infty} \frac{g_{2}^{(l+1)}(x)}{\left(g_{2}-g_{1}\right)^{(l)}(x)}$ exists in $\mathbf{R} \cup\{ \pm \infty\}$. Let $h \in \mathbf{N}$, and let

$$
g(x)=g_{2}(x+h)-g_{1}(x) .
$$


Then for sufficiently large $h$,

$$
g \in \mathcal{G}(\lambda), \text { where } \lambda= \begin{cases}\alpha_{2} & \text { if } \alpha_{2}>\alpha_{1} \\ \max \left\{\alpha_{2}-1, \beta\right\} & \text { if } \alpha_{2}=\alpha_{1}, g_{2}-g_{1} \in \mathcal{G}(\beta), \beta \leq \alpha_{2} .\end{cases}
$$

If $\alpha_{2} \in \mathbf{N}$ and $g_{2}-g_{1} \in \mathcal{G}\left(\alpha_{2}-1\right)$ then $g \in \mathcal{P}$ if either $g_{2}-g_{1}$ or $g_{2}$ is in $\mathcal{P}$.

Proof: If $\alpha_{1}<\alpha_{2}$, then $g_{2}-g_{1} \in \mathcal{G}\left(\alpha_{2}\right)$ by Lemma 5.6 and $\Delta_{h} g_{2} \in \mathcal{G}\left(\alpha_{2}-1\right)$ by Lemma 5.7 so that $g=g_{2}-g_{1}+\Delta_{h} g_{2} \in \mathcal{G}\left(\alpha_{2}\right)$ by Lemma 5.6.

Suppose that $\alpha_{1}=\alpha_{2}=\alpha$ and that $g_{2}-g_{1} \in \mathcal{G}(\beta)$, where $\beta \leq \alpha$. If $\beta \neq \alpha-1$, then $g=g_{2}-g_{1}+\Delta_{h} g_{2} \in \mathcal{G}(\lambda)$, where $\lambda=\max \{\beta, \alpha-1\}$, by Lemma 5.6.

Suppose that $\beta=\alpha-1$. Since $g_{2}-g_{1}, \Delta_{h} g_{2} \in \mathcal{G}(\alpha-1)$, it follows by Proposition 5.4 that there exist $f_{21}, \tilde{f}_{2} \in \mathcal{S}$ such that $g_{2}-g_{1}=x^{\alpha-1} f_{21}$ and $\Delta_{h} g_{2}=x^{\alpha-1} \tilde{f}_{2}$. Let $f=f_{21}+\tilde{f}_{2}$ so that $g=x^{\alpha-1} f$. By Lemma 5.5(v), $f \in \mathcal{S}$ if $\lim _{x \rightarrow \infty} \frac{\tilde{f}_{2}(x)}{f_{21}(x)}$ exists in $\mathbf{R} \cup\{ \pm \infty\}$ and does not equal -1 . If $\alpha \in \mathbf{N}$ then there exist $\gamma_{21}, \gamma_{2} \in \mathbf{R}$ such that $\lim _{x \rightarrow \infty} f_{21}(x)=\gamma_{21}$ and $\lim _{x \rightarrow \infty} \tilde{f}_{2}(x)=\gamma_{2} h$. Hence, $f \in \mathcal{S}$ and $\lim _{x \rightarrow \infty} f(x) \neq 0$ if either $g_{2}-g_{1} \in \mathcal{P}$ or $g_{2} \in \mathcal{P}$ so that $g \in \mathcal{P}$ in these cases by Proposition 5.4.

Suppose now that $g_{2}, g_{2}-g_{1} \in \mathcal{T}$. We have for some $x<z_{x}<x+h$ and by Lemma 2.3 that

$$
\lim _{x \rightarrow \infty} \frac{\tilde{f}_{2}(x)}{f_{21}(x)}=\lim _{x \rightarrow \infty} \frac{\Delta_{h} g_{2}(x)}{g_{2}(x)-g_{1}(x)}=\lim _{x \rightarrow \infty} \frac{g_{2}^{\prime}\left(z_{x}\right) h}{g_{2}(x)-g_{1}(x)}=\lim _{x \rightarrow \infty} \frac{g_{2}^{\prime}(x) h}{g_{2}(x)-g_{1}(x)},
$$

which exists since by our assumption and Lemma $5.8, \lim _{x \rightarrow \infty} \frac{g_{2}^{(i+1)}(x)}{\left(g_{2}-g_{1}\right)^{(i)}(x)}$ exists in $\mathbf{R} \cup\{ \pm \infty\}$ for all $i \geq 0$, and is different from -1 for sufficiently large $h$. Hence, if $\alpha \notin \mathbf{N}$ then $g \in \mathcal{T}(\alpha-1)$ by Proposition 5.4. It is left to prove that $g \in \mathcal{R}$ if $\alpha \in \mathbf{N}$.

Let $i \in \mathbf{N}$. If $\lim _{x \rightarrow \infty} \frac{g_{2}^{(i+1)}(x)}{\left(g_{2}-g_{1}\right)^{(i)}(x)} \in \mathbf{R}$ then

$$
\lim _{x \rightarrow \infty} \frac{x g^{(i+1)}(x)}{g^{(i)}(x)}=\lim _{x \rightarrow \infty} \frac{\frac{x\left(g_{2}-g_{1}\right)^{(i+1)}(x)}{\left(g_{2}-g_{1}\right)^{(i)}(x)}+h \frac{x g_{2}^{(i+2)}(x)}{g_{2}^{(i+1)}(x)} \frac{g_{2}^{(i+1)}(x)}{\left(g_{2}-g_{1}\right)^{(i)}(x)}}{1+h \frac{g_{2}^{(i+1)}(x)}{\left(g_{2}-g_{1}\right)^{(i)}(x)}}
$$

exists since the limits of all the smaller fractions exist. Hence, $g \in \mathcal{R}$ and therefore $g \in \mathcal{T}(\alpha-1)$. The case where $\lim _{x \rightarrow \infty} \frac{g_{2}^{(i+1)}(x)}{\left(g_{2}-g_{1}\right)^{(i)}(x)} \in\{ \pm \infty\}$, is similar.

The following corollary now follows from the lemmas 5.7 and 5.9 and easy checking.

Corollary 5.10 Let $G \subset \bigcup_{i>1} \mathcal{G}_{i}$ be such that $G_{\text {diff }}=\left\{g_{1}-g_{2} \mid g_{1}, g_{2} \in G, g_{1} \neq g_{2}\right\} \subset \mathcal{G}$. Suppose that if $\alpha>0,[\alpha]=\bar{l}$, and $g_{1}, g_{2} \in G \cap \mathcal{T}(\alpha)$ are such that $g_{2}-g_{1} \in \mathcal{T}(\alpha-1)$, then $\lim _{x \rightarrow \infty} \frac{g_{2}^{(l+1)}(x)}{\left(g_{2}-g_{1}\right)^{(l)}(x)}$ exists in $\mathbf{R} \cup\{ \pm \infty\}$. Then for any $g_{1} \in G$ and for all sufficiently large $h \in \mathbf{N}$, the set

$$
\hat{G}=\left\{g-g_{1} \mid g \in G, g \neq g_{1}\right\} \cup\left\{\hat{g} \mid \hat{g}(x)=g(x+h)-g_{1}(x), g \in G\right\}
$$

is contained in $\mathcal{G}$ and $\hat{G}_{\text {diff }}=\left\{g_{1}-g_{2} \mid g_{1}, g_{2} \in \hat{G}, g_{1} \neq g_{2}\right\} \subset \mathcal{G}$. 
Proposition 5.11 Let $G \subset \mathcal{G}$ be a finite subset such that $G_{\text {diff }}=\left\{g_{1}-g_{2} \mid g_{1}, g_{2} \in G, g_{1} \neq\right.$ $\left.g_{2}\right\} \subset \mathcal{G}$ and suppose that $G$ has the R-property.

(i) If $G \subset \mathcal{G}_{0}$ then $G$ has the M-property.

(ii) Suppose that $G \subset \bigcup_{i \geq 1} \mathcal{G}_{i}$. If $g_{1} \in G$ is such that $\frac{g_{1}}{g}$ is bounded for all $g \in G$, and if $h \in \mathbf{N}$, let

$$
\hat{G}=\left\{g-g_{1} \mid g \in G, g \neq g_{1}\right\} \cup\left\{\hat{g} \mid \hat{g}(x)=g(x+h)-g_{1}(x), g \in G\right\} .
$$

Then for sufficiently large $h, \hat{G} \cup \hat{G}_{\text {diff }} \subset \mathcal{G}$ and $\hat{G}$ has the R-property.

Proof: (i). Let $G \subset \mathcal{G}_{0}, 0<\alpha \leq 1, G(\alpha)=G \cap \mathcal{G}(\alpha)$ and let $\psi_{1}, \psi_{2} \in\left(G(\alpha) \cup G(\alpha)_{\text {diff }}\right) \cap \mathcal{T}$. Suppose that $\psi_{1} \in \mathcal{T}\left(\beta_{1}\right)$ and $\psi_{2} \in \mathcal{T}\left(\beta_{2}\right)$. If $\beta_{1}>\beta_{2}$ and $f=\frac{\psi_{2}^{\prime}}{\psi_{1}^{\prime}}$, then by Lemma 2.6, $f$ goes monotonically to 0 , and $\lim _{x \rightarrow \infty} \frac{x f^{\prime}}{f}=\beta_{2}-\beta_{1} \neq 0$. If $\beta_{1}=\beta_{2}$ then by the R-property, $\lim _{x \rightarrow \infty} \frac{\psi_{1}^{\prime}}{\psi_{2}^{\prime}} \in \mathbf{R} \backslash\{0\}$. Hence, $G$ has the M-property.

(ii). Since $G$ has the R-property, $G$ satisfies the conditions of Corollary 5.10 such that for sufficiently large $h, \hat{G} \cup \hat{G}_{\text {diff }} \subset \mathcal{G}$. It is left to prove that $G$ has the R-property.

Let $g_{1} \in G \cap \mathcal{G}\left(\alpha_{1}\right)$, where $\alpha_{1}>0$, and let $\alpha>0$. Any function in $\hat{G}(\alpha)$ is of the form $g_{2}-g_{1}$ or $g_{2}-g_{1}+\Delta_{h} g_{2}$, where $g_{2} \in G \cap \mathcal{G}\left(\alpha_{2}\right)$, and since $\alpha_{1} \leq \alpha_{2}$, either $\alpha_{1}<\alpha=\alpha_{2}$ or $\alpha_{1}=\alpha_{2} \geq \alpha$. Therefore, any function in $\hat{G}(\alpha) \cup \hat{G}(\alpha)_{\text {diff }}$ is of one of the forms

$$
g_{2}-g_{1}, \quad g_{3}-g_{1}+\Delta_{h} g_{3}, \quad g_{4}-g_{5}, \quad g_{6}-g_{7}+\Delta_{h} g_{6}, \quad g_{8}-g_{9}+\Delta_{h}\left(g_{8}-g_{9}\right)
$$

where $g_{i} \in G \cap \mathcal{G}\left(\alpha_{i}\right), i=2, \ldots, 9$ and either $\alpha_{1}<\alpha_{2}=\cdots=\alpha_{9}=\alpha$ or $\alpha_{1}=\alpha_{2}=\cdots=\alpha_{9} \geq$ $\alpha$. If $\alpha_{1}<\alpha$ then the ratios under study have good behavior by the R-property of $G$. Suppose now that $\alpha_{1} \geq \alpha$. Let us for now say that a function $g$ has the rate $\beta$ if $g \in \mathcal{G}(\beta)$.

For each $i \in\{1,2\}$, let $\psi_{i} \in \mathcal{T}\left(\beta_{i}\right)$ be a function of one of the forms (26), and suppose that $\beta_{1}-\beta_{2}=l \in \mathbf{N} \cup\{0\}$ and that $\psi_{1}^{(l+k)}$ and $\psi_{2}^{(k)}$ have the same rate $\beta$, where $k=\left[\beta_{2}\right]$. Then $\frac{\psi_{1}^{(l+k)}}{\psi_{2}^{(k)}}$ may be written as a ratio of sums of partial fractions where the common denominator of each partial fraction has the rate $\beta$, as in the following example,

$$
\lim _{x \rightarrow \infty} \frac{\psi_{1}^{(l+k)}}{\psi_{2}^{(k)}}=\lim _{x \rightarrow \infty} \frac{\left(g_{3}-g_{1}\right)^{(l+k)}}{\left(g_{6}-g_{7}+\Delta_{h} g_{6}\right)^{(k)}}=\lim _{x \rightarrow \infty} \frac{\frac{\left(g_{3}-g_{1}\right)^{(l+k)}}{g_{6}^{(k+1)}}}{\frac{\left(g_{6}-g_{7}\right)^{(k)}}{g_{6}^{(k+1)}}+h},
$$

where $\left(g_{3}-g_{1}\right)^{(l+k)}, g_{6}^{(k+1)} \in \mathcal{T}(\beta)$ and $\left(g_{6}-g_{7}\right)^{(k)} \in \mathcal{G}(\lambda), \lambda \leq \beta$. Note that $\Delta_{h} g$ has the same asymptotic behavior as $h g^{\prime}$. If the numerator of the partial fraction has lower rate than $\beta$ then this partial fraction tends to 0 . Otherwise, it tends to a non-zero number by the R-property of $G$. It follows that by letting $h$ be sufficiently large, $\lim _{x \rightarrow \infty} \frac{\psi_{1}^{(l+k)}}{\psi_{2}^{(k)}} \in \mathbf{R} \backslash\{0\}$.

The following lemma will be used in Section 7. Recall the definition of tempered functions, Definition 1.4. 
Lemma 5.12 If $g(x)$ is a tempered function then $[g(n)] \gamma, n=1,2, \ldots$, is uniformly distributed (mod 1) if and only if $\gamma$ is irrational.

Proof: By Fejér's theorem, Theorem 1.5, and the remark following it, $g(n) \lambda, n=1,2, \ldots$, is uniformly distributed $(\bmod 1)$ for any $\lambda \in \mathbf{R} \backslash\{0\}$. Let $h \in \mathbf{Z} \backslash\{0\}$. Then

$$
\lim _{N \rightarrow \infty} \frac{1}{N} \sum_{n=1}^{N} e^{2 \pi i h[g(n)] \gamma}=\lim _{N \rightarrow \infty} \frac{1}{N} \sum_{n=1}^{N} e^{2 \pi i h(g(n) \gamma-\{g(n)\} \gamma)}=\lim _{N \rightarrow \infty} \frac{1}{N} \sum_{n=1}^{N} f(g(n) \gamma, g(n)),
$$

where $f(x, y)=e^{2 \pi i h(x-\{y\} \gamma)}$ is a Riemann-integrable periodic mod 1 function on $\mathbf{R}^{2}$. So if $(g(n) \gamma, g(n))$ is uniformly distributed $(\bmod 1)$ in $\mathbf{R}^{2}$ then $\lim _{N \rightarrow \infty} \frac{1}{N} \sum_{n=1}^{N} e^{2 \pi i h[g(n)] \gamma}=$ $\int_{0}^{1} \int_{0}^{1} f(x, y) d x d y=0$ and by Weyl criterion (see [19, p. 7]), $[g(n)] \gamma, n=1,2, \ldots$, is uniformly distributed $(\bmod 1)$. Now, $(g(n) \gamma, g(n))$ is uniformly distributed $(\bmod 1)$ in $\mathbf{R}^{2}$ if and only if $a g(n) \gamma+b g(n)=(a \gamma+b) g(n)$ is uniformly distributed $(\bmod 1)$ for all $a, b \in \mathbf{Z},(a, b) \neq(0,0)$, which is true if and only if $\gamma$ is irrational.

We will end this section by proving a convergence theorem for tempered functions (and not just for functions in the class $\mathcal{T}$ ).

Let $l \in \mathbf{N}$ and for $0 \leq i \leq l$, let $\psi_{i}$ be a tempered function of order $i$, and let

$$
\mathcal{E}\left(\psi_{1}, \ldots, \psi_{l}\right)=\operatorname{span}\left\{\psi_{i}^{(j)} \mid 0 \leq j \leq i, i=0, \ldots, l\right\} \backslash\{0\},
$$

where the linear span is taken over $\mathbf{R}$. We will say that $g \in \mathcal{E}\left(\psi_{1}, \ldots, \psi_{l}\right)$ has order $i$ if $g=\sum_{j=1}^{r} g_{j}$, where $g_{j} \in \mathcal{E}\left(\psi_{1}, \ldots, \psi_{l}\right)$ is tempered, and $\max _{j}\left\{\operatorname{order}\left(g_{j}\right)\right\}=i$.

A subset $\left\{g_{1}, \ldots, g_{k}\right\} \subset \mathcal{E}\left(\psi_{1}, \ldots, \psi_{l}\right)$ is said to be admissible if for any $1 \leq i<j \leq k$ one of the following (mutually exclusive) conditions holds:

(i) the order of $g_{i}$ is less than the order of $g_{j}$

(ii) there exists $c \in \mathbf{R} \backslash\{0\}$ and $1 \leq r \leq \operatorname{order}\left(g_{j}\right)$ such that $g_{j}-g_{i}=c g_{j}^{(r)}+p_{1}, g_{j}^{(r)}, p_{1} \in$ $\mathcal{E}\left(\psi_{1}, \ldots, \psi_{l}\right) \cup\{0\}$ and $\frac{p_{1}}{g_{j}^{(r)}} \rightarrow 0$

(iii) there exists $d \in \mathbf{R} \backslash\{0,1\}$ such that $g_{j}-g_{i}=d g_{j}+p_{2}, p_{2} \in \mathcal{E}\left(\psi_{1}, \ldots, \psi_{l}\right) \cup\{0\}$ and $\frac{p_{2}}{g_{j}} \rightarrow 0$.

Example: The set $\left\{\log ^{3 / 2} x, 2 \log ^{3 / 2} x, x \log ^{3 / 2} x, x^{5 / 2} \log x, x^{\pi}(2+\cos \sqrt{\log x})\right\}$ is admissible. See also additional examples of admissible sets in the formulation of Corollary 5.14 below.

Theorem 5.13 Let $l \in \mathbf{N}$, and let $\psi_{1}, \ldots, \psi_{l}$ and $\mathcal{E}\left(\psi_{1}, \ldots, \psi_{l}\right)$ be defined as in (27) above. Let $k \in \mathbf{N}$, and suppose that $\left\{g_{1}, \ldots, g_{k}\right\} \subset \mathcal{E}\left(\psi_{1}, \ldots, \psi_{l}\right)$ is admissible. Then for any invertible weakly mixing system $(X, \mathcal{B}, \mu, T)$ and any $f_{1}, \ldots, f_{k} \in L^{\infty}(X, \mathcal{B}, \mu)$,

$$
\lim _{N \rightarrow \infty}\left\|\frac{1}{N} \sum_{n=1}^{N} T^{\left[g_{1}(n)\right]} f_{1} \cdots T^{\left[g_{k}(n)\right]} f_{k}-\prod_{i=1}^{k} \int f_{i}\right\|_{2}=0
$$


Proof: Let first $l=0$ and $\psi$ a Fejér function. Then $\mathcal{E}(\psi)=\{c \psi \mid c \neq 0\}$ and $g_{i}=c_{i} \psi$, $i=1, \ldots, k$, such that $c_{i} \neq c_{j}$ when $i \neq j$. Consider

$$
\frac{1}{N} \sum_{n=1}^{N} T^{\left[c_{1} \psi(n)\right]} f_{1} \cdots T^{\left[c_{k} \psi(n)\right]} f_{k},
$$

where we may assume that $\int f_{1}=0$. By making a change of variables, $m=[\psi(n)]$, we have $\left[c_{i} \psi_{i}(n)\right]=\left[c_{i} m\right]+d_{i}(n)$, where $d_{i}(n)$ takes only finitely many values. Let $\phi(n)=\mid\{k \in \mathbf{N} \mid$ $[\psi(k)]=n\} \mid$ such that by the Lemmas 2.4 and 2.5, $\phi(n)$ is almost increasing (see Remark after Theorem 3.6) and $\lim _{n \rightarrow \infty} \frac{\phi(n)}{\Phi(n)}=0$, where $\Phi(n)=\sum_{k=0}^{n} \phi(k)$. Then by Theorem 3.6 and Proposition 4.4,

$$
\begin{aligned}
\lim _{N \rightarrow \infty}\left\|\frac{1}{N} \sum_{n=1}^{N} T^{\left[c_{1} \psi(n)\right]} f_{1} \cdots T^{\left[c_{k} \psi(n)\right]} f_{k}\right\|_{2} & =\lim _{N \rightarrow \infty}\left\|\frac{1}{\Phi(N)} \sum_{n=0}^{N} \phi(n) T^{\left[c_{1} n\right]+d_{1}} f_{1} \cdots T^{\left[c_{k} n\right]+d_{k}} f_{k}\right\|_{2} \\
& =\lim _{N \rightarrow \infty}\left\|\frac{1}{N} \sum_{n=1}^{N} T^{\left[c_{1} n\right]+d_{1}} f_{1} \cdots T^{\left[c_{k} n\right]+d_{k}} f_{k}\right\|_{2}=0 .
\end{aligned}
$$

The rest of the proof, when $l>0$, goes along the same lines as the proof of Theorem $\mathrm{B}$ in Section 6 below and the details are left to the reader. However, we will here check that when using the van der Corput trick, we get a new set of functions which also is admissible. First, notice that if $\theta_{1}, \theta_{2}$ are two functions and $\theta_{2} \rightarrow 0$ then $\left[\theta_{1}(n)+\theta_{2}(n)\right]=\left[\theta_{1}(n)\right]+d_{n}$, where $d_{n}$ takes only finitely many values, and therefore $\theta_{2}$ may be neglected. Hence, if $g$ has order $i$ and $\Delta_{h} g-\left(h g^{\prime}+\sum_{j=2}^{i} a_{h} g^{(j)}\right) \rightarrow 0$ then we can use $\tilde{\Delta}_{h} g=h g^{\prime}+\sum_{j=2}^{i} a_{h} g^{(j)}$ instead of $\Delta_{h} g$. Assume that $g_{1}$ has order at least one, and that $\operatorname{order}\left(g_{i}\right) \geq \operatorname{order}\left(g_{1}\right), i=2, \ldots, k$. We will show that the set

$$
\hat{G}=\left\{\tilde{\Delta}_{h} g_{1}, g_{i}-g_{1}, g_{i}-g_{1}+\tilde{\Delta}_{h} g_{i} \mid i=2, \ldots, k\right\},
$$

which is a subset of $\mathcal{E}\left(\psi_{1}, \ldots, \psi_{l}\right)$, is admissible. We have that

- the pair $g_{i}-g_{1}+\tilde{\Delta}_{h} g_{i}$ and $g_{i}-g_{1}$ satisfies condition (ii) of the definition of admissible sets (see above) with $r=1$ if the pair $g_{1}, g_{i}$ satisfies the condition (i) or (iii), and it satisfies the condition (iii) if the pair $g_{1}, g_{i}$ satisfies the condition (ii) with $r=1$, and (i) if $g_{1}, g_{i}$ satisfies (ii) with $r>1$,

- the pair $g_{i}-g_{1}$ and $\tilde{\Delta}_{h} g_{1}$ satisfies the condition (i) if $g_{i}-g_{1}$ has the same order as $g_{i}$ or has smaller order than $g_{i}^{\prime}$, and the condition (iii) if $g_{i}, g_{1}$ satisfies (ii) with $r=1$,

- the pair $g_{i}-g_{1}+\tilde{\Delta}_{h} g_{i}$ and $\tilde{\Delta}_{h} g_{1}$ satisfies the condition (i) if $g_{i}-g_{1}$ has the same order as $g_{i}$, and the condition (iii) if $g_{i}, g_{1}$ satisfies (ii),

- if $i>j, \epsilon_{i}, \epsilon_{j} \in\{0,1\}$ and $\omega_{s}=g_{s}-g_{1}+\epsilon_{s} \tilde{\Delta}_{h} g_{s}, s=i, j$, the pair $\omega_{i}, \omega_{j}$ satisfies the same condition as $g_{i}, g_{j}$ does if $\operatorname{order}\left(g_{1}\right)<\operatorname{order}\left(g_{j}\right) \leq \operatorname{order}\left(g_{i}\right)$ or $\operatorname{order}\left(g_{j}\right)<\operatorname{order}\left(g_{i}\right)$. Suppose that $\operatorname{order}\left(g_{1}\right)=\operatorname{order}\left(g_{i}\right)=\operatorname{order}\left(g_{j}\right)$. Then for $s=i, j, g_{s}=g_{1}+c_{s} g_{1}^{\left(r_{s}\right)}+p_{s}$, where $r_{s} \geq 0$ and $\frac{p_{s}}{g_{1}^{\left(r_{s}\right)}} \rightarrow 0$. Hence, since $\omega_{i}-\omega_{j}$ has at least order 0 , the pair $\omega_{i}, \omega_{j}$ satisfies (i), (ii) or (iii). 
Hence, $\hat{G}$ is admissible.

Corollary 5.14 Let $g_{1}, \ldots, g_{k}$ be tempered functions and assume that they satisfy one of the following four conditions.

(a) $g_{i}=a_{i} g, i=1, \ldots, k$, where $g$ is a tempered function and $a_{1}<a_{2}<\cdots<a_{k}$.

(b) $g_{i}(x)=g\left(x+a_{i}\right), i=1, \ldots, k$, where $g$ is tempered of order at least one and $0 \leq a_{1}<$ $a_{2}<\cdots<a_{k}$.

(c) $g_{i}$ is tempered of order $i, i=1, \ldots, k$.

(d) If $l \in \mathbf{N}$ and $\phi$ is tempered of order $l$, let $\Omega=\left\{\sum_{i=0}^{l} a_{i} \phi^{(i)} \mid a_{i} \in \mathbf{R}\right\} \backslash\{0\}$, and let $g_{1}, \ldots, g_{k} \in \Omega$ be such that $g_{i}-g_{j} \in \Omega$ for all $i \neq j$.

Then for any invertible weakly mixing system $(X, \mathcal{B}, \mu, T)$ and any $f_{1}, \ldots, f_{k} \in L^{\infty}(X, \mathcal{B}, \mu)$,

$$
\lim _{N \rightarrow \infty}\left\|\frac{1}{N} \sum_{n=1}^{N} T^{\left[g_{1}(n)\right]} f_{1} \cdots T^{\left[g_{k}(n)\right]} f_{k}-\prod_{i=1}^{k} \int f_{i}\right\|_{2}=0 .
$$

\section{Remark:}

A natural extension of (a) could possibly involve powers of a tempered function $g$. However, a power of a tempered function does not need to be tempered and may not be an element of $\mathcal{G}$ even if $g \in \mathcal{T}$. For example, if $g(x)=\sqrt{x}(2+\cos \sqrt{\log x})$ then $g \in \mathcal{T}_{0}$ but $g^{2}(x)=$ $x(2+\cos \sqrt{\log x})^{2} \notin \mathcal{G}$.

\section{Proofs of Theorem A and Theorem B}

The proofs of Theorems A and B are similar and go along the lines of the proof of the weakly mixing PET in [2]. We will sketch here the proof of Theorem B; the reader should have no problem to check that an almost identical argument gives a proof of Theorem A.

Let $G=\left\{g_{1}, \ldots, g_{k}\right\}$ be a finite subset of $\mathcal{G}$ such that $g_{i}-g_{j} \in \mathcal{G}$ for $i \neq j$ and such that $G$ has the R-property.

Suppose that $d=\max \left\{i \mid G \cap \mathcal{G}_{i} \neq \emptyset\right\}$. Let $G_{i}=G \cap \mathcal{G}_{i}, i=0,1, \ldots, d$, so that $G=\bigcup_{i=0}^{d} G_{i}$, where $G_{d} \neq \emptyset$. Each $G_{i}$ can be further decomposed into the disjoint union

$$
G_{i}=\bigcup_{j=1}^{n_{i}} G_{j}^{(i)}
$$

where $G_{j}^{(i)}, j=1, \ldots, n_{i}$, have the following property: if $g_{r_{1}} \in G_{r}^{(i)}$ and $g_{s_{1}} \in G_{s}^{(i)}$ then $r=s$ if and only if $g_{r_{1}}-g_{s_{1}} \notin \mathcal{G}_{i}$. So we have

$$
G=\bigcup_{i=0}^{d} \bigcup_{j=1}^{n_{i}} G_{j}^{(i)}
$$


We say that $G$ has the characteristic vector $\left(n_{0}, n_{1}, \ldots, n_{d}\right)$, where $n_{i}$ is the number of different groups of functions in $G_{i}$.

Example: The functions $g_{1}=x^{3 / 2}, g_{2}=x^{3 / 2}(3+\cos \sqrt{\log x}), g_{3}=x^{3 / 2}+x^{5 / 4}, g_{4}=x^{3 / 2}+x^{1 / 2}$, are in $G_{1}$ and are divided into the three groups $\left\{g_{1}, g_{4}\right\},\left\{g_{2}\right\}$ and $\left\{g_{3}\right\}$. Hence, $n_{1}=3$.

Let $\mathcal{F}\left(n_{0}, n_{1}, \ldots, n_{d}\right)$ be the family of all finite subsets $G \subset \mathcal{G}$ with the following two properties:

(i) $G$ has the characteristic vector $\left(n_{0}, n_{1}, \ldots, n_{d}\right)$.

(ii) $G$ satisfies the conditions of Theorem B.

Consider the following two statements:

$T\left(n_{0}, n_{1}, \ldots, n_{d}\right)$ : 'Theorem $\mathrm{B}$ is valid for any $G \in \mathcal{F}\left(n_{0}, n_{1}, \ldots, n_{d}\right)$.'

$T\left(\overline{n_{0}, \ldots, n_{i}}, n_{i+1}, \ldots, n_{d}\right)$ : ' $T\left(n_{0}, n_{1}, \ldots, n_{d}\right)$ is valid for any $\left(n_{0}, n_{1}, \ldots, n_{i}\right)$.'

Note that Theorem B is equivalent to the statement: ' $T\left(\overline{n_{0}, n_{1}, \ldots, n_{d}}\right)$ is valid for any $d$ '. It is enough (see the proof of [2, Theorem 1.2]) to prove the case $d=0$ and $n_{0}=1$ and the following implications:

$$
\begin{gathered}
T\left(n_{0}, n_{1}, \ldots, n_{d}\right) \Rightarrow T\left(n_{0}+1, n_{1}, \ldots, n_{d}\right) ; n_{0}, \ldots, n_{d-1} \geq 0, n_{d} \geq 1, d \geq 0 \\
T\left(\overline{n_{0}, \ldots, n_{i-1}}, n_{i}, \ldots, n_{d}\right) \Rightarrow \quad T(\underbrace{0, \ldots, 0}_{i \text { zeros }}, n_{i}+1, n_{i+1}, \ldots, n_{d}), \\
n_{i}, \ldots, n_{d-1} \geq 0, n_{d} \geq 1, d \geq i>0 \\
T\left(\overline{n_{0}, n_{1}, \ldots, n_{d}}\right) \Rightarrow T(\underbrace{0, \ldots, 0}_{d+1 \text { zeros }}, 1), d \geq 1
\end{gathered}
$$

The case $d=0$ and $n_{0}=1$ and the implication (28) for $d=0$ is done in Theorem 4.3. The proof of (28) for $d>0$ can be done similarly. The proof of (30) is the same as that of (29). Thus we will finish the proof by proving (29).

Suppose that $G$ is a finite set of functions from $\mathcal{G}$ such that also the difference of any pair of them is in $\mathcal{G}$, and such that $G$ has the R-property, and assume that $G$ has the characteristic vector $\left(0, \ldots, 0, n_{i}+1, n_{i+1}, \ldots, n_{d}\right)$, where $i>0$. Fix one of the $n_{i}+1$ groups of functions from $G_{i}$ such that if $g_{11}, \ldots, g_{1 k}$ are its functions and the rest of the functions in $G$ is denoted by $g_{21}, \ldots, g_{2 l}$, then $\frac{g_{11}}{g_{2 j}}$ is bounded for each $j$. Let

$$
x_{n}=T^{\left[g_{11}(n)\right]} f_{11} \cdots T^{\left[g_{1 k}(n)\right]} f_{1 k} T^{\left[g_{21}(n)\right]} f_{21} \cdots T^{\left[g_{2 l}(n)\right]} f_{2 l},
$$

where $f_{i j} \in L^{\infty}(X, \mathcal{B}, \mu)$. Without loss of generality we may assume that one of the functions $f_{i j}$ has zero integral. With this assumption we have to show that $\lim _{N \rightarrow \infty}\left\|\frac{1}{N} \sum_{n=1}^{N} x_{n}\right\|_{2}=0$. By Theorem 4.1 it is enough to prove that

$$
\limsup _{N \rightarrow \infty} \frac{1}{N} \sum_{n=1}^{N}\left\langle x_{n+h}, x_{n}\right\rangle=0
$$


for all sufficiently large $h$. We have

$$
\begin{aligned}
& \left\langle x_{n+h}, x_{n}\right\rangle \\
& =\int \prod_{j=1}^{k} T^{\left[g_{1 j}(n+h)\right]} f_{1 j} \prod_{j=1}^{l} T^{\left[g_{2 j}(n+h)\right]} f_{2 j} \prod_{j=1}^{k} T^{\left[g_{1 j}(n)\right]} f_{1 j} \prod_{j=1}^{l} T^{\left[g_{2 j}(n)\right]} f_{2 j} d \mu \\
& =\int T^{\left[g_{11}(n)\right]}\left(f_{11} \prod_{j=1}^{k} T^{\left[g_{1 j}(n+h)\right]-\left[g_{11}(n)\right]} f_{1 j} \prod_{j=1}^{l} T^{\left[g_{2 j}(n+h)\right]-\left[g_{11}(n)\right]} f_{2 j}\right. \\
& \left.\quad \times \prod_{j=2}^{k} T^{\left[g_{1 j}(n)\right]-\left[g_{11}(n)\right]} f_{1 j} \prod_{j=1}^{l} T^{\left[g_{2 j}(n)\right]-\left[g_{11}(n)\right]} f_{2 j}\right) d \mu \\
& =\int f_{11} \prod_{j=1}^{k} T^{\left[\hat{g}_{1 j}(n)\right]+c_{1 j}} f_{1 j} \prod_{j=1}^{l} T^{\left[\hat{\bar{g}}_{2 j}(n)\right]+c_{2 j}} f_{2 j} \prod_{j=2}^{k} T^{\left[\hat{g}_{1 j}(n)\right]+c_{3 j}} f_{1 j} \prod_{j=1}^{l} T^{\left[\hat{g}_{2 j}(n)\right]+c_{4 j}} f_{2 j} d \mu
\end{aligned}
$$

where $c_{i j} \in\{0,1\}$ and

$$
\begin{aligned}
& \hat{g}_{1 j}(n)=g_{1 j}(n)-g_{11}(n), j=2, \ldots, k \\
& \hat{g}_{2 j}(n)=g_{2 j}(n)-g_{11}(n), j=1,2, \ldots, l \\
& \hat{\bar{g}}_{1 j}(n)=g_{1 j}(n+h)-g_{11}(n), j=1,2, \ldots, k \\
& \hat{\bar{g}}_{2 j}(n)=g_{2 j}(n+h)-g_{11}(n), j=1,2, \ldots, l .
\end{aligned}
$$

By Lemma 3.4 we may treat the $c_{i j}$ as constants. By Proposition 5.11 the set $\hat{G}=\left\{\hat{g}_{1 j} \mid\right.$ $j=2, \ldots, k\} \cup\left\{\hat{g}_{2 j} \mid j=1, \ldots, l\right\} \cup\left\{\hat{\bar{g}}_{1 j} \mid j=1, \ldots, k\right\} \cup\left\{\hat{\bar{g}}_{2 j} \mid j=1, \ldots, l\right\} \subset \mathcal{G}$ and is such that any pairwise differences of functions from $\hat{G}$ are in $\mathcal{G}$ for all sufficiently large $h$, and $\hat{G}$ has the R-property. By Lemma 5.9, the characteristic vector of the set $\left\{\hat{g}_{12}(n), \ldots, \hat{g}_{1 k}(n), \hat{\bar{g}}_{11}(n), \ldots, \hat{\bar{g}}_{1 k}(n), \hat{g}_{21}(n), \ldots, \hat{g}_{2 l}(n), \hat{\bar{g}}_{21}(n), \ldots, \hat{\bar{g}}_{2 l}(n)\right\}$ has the form $\left(a_{0}, a_{1}, \ldots, a_{i-1}, n_{i}, n_{i+1}, \ldots, n_{d}\right)$. Applying $T\left(\overline{n_{0}, \ldots, n_{i-1}}, n_{i}, \ldots, n_{d}\right)$ (weak convergence) and using the fact that one of the functions $f_{i j}$ has zero integral, we get

$$
\lim _{N \rightarrow \infty} \frac{1}{N} \sum_{n=1}^{N}\left\langle x_{n+h}, x_{n}\right\rangle=\prod_{j=1}^{k} \int f_{1 j} d \mu \prod_{j=1}^{l} \int f_{2 j} d \mu \prod_{j=1}^{k} \int f_{1 j} d \mu \prod_{j=1}^{l} \int f_{2 j} d \mu=0 .
$$

\section{$7 \quad$ Ergodicity of higher orders for Fejér functions}

We start this section by proving a result which is an extension of the case $k=1$ of Theorems A, B and 4.3, in two directions. First, it holds for all tempered functions and not just for some subclass thereof. Second, it holds for any ergodic system.

Theorem 7.1 If $g$ is a tempered function, $(X, \mathcal{B}, \mu, T)$ is an invertible ergodic system and $f \in L^{2}(X, \mathcal{B}, \mu)$, then

$$
\lim _{N \rightarrow \infty}\left\|\frac{1}{N} \sum_{n=1}^{N} T^{[g(n)]} f-\int f\right\|_{2}=0
$$


Proof: We may assume that $\int f=0$. According to Lemma 5.12, $[g(n)] \gamma, n=1,2, \ldots$, is uniformly distributed $(\bmod 1)$ for any irrational $\gamma$. Hence, since

$$
\lim _{N \rightarrow \infty} \frac{1}{N} \sum_{n=1}^{N} e^{2 \pi i[g(n)] \gamma}=0
$$

for any $\gamma \in \mathbf{Q} \backslash \mathbf{Z}$ by [19, Theorem 1.4, p. 307], (32) is true for any $\gamma \in(0,1)$. By using the spectral theorem, we have

$$
\begin{aligned}
\left\|\frac{1}{N} \sum_{n=1}^{N} T^{[g(n)]} f\right\|_{2}^{2} & =\frac{1}{N^{2}} \sum_{n, m=1}^{N} \int T^{[g(n)]} f T^{[g(m)]} f d \mu \\
& =\frac{1}{N^{2}} \sum_{n, m=1}^{N} \int f T^{[g(n)]-[g(m)]} f d \mu \\
& =\frac{1}{N^{2}} \sum_{n, m=1}^{N} \int e^{2 \pi i([g(n)]-[g(m)]) \gamma} d \nu_{f}(\gamma) \\
& =\int\left|\frac{1}{N} \sum_{n=1}^{N} e^{2 \pi i[g(n)] \gamma}\right|^{2} d \nu_{f}(\gamma)
\end{aligned}
$$

where $\nu_{f}$ is the spectral measure with $\nu_{f}(\{0\})=0$ since $T$ is ergodic and $\int f=0$. Hence, (33) converges to 0 as $N \rightarrow \infty$.

Corollary 7.2 If $g$ is a tempered function, $(X, \mathcal{B}, \mu, T)$ an invertible measure preserving system and $A \in \mathcal{B}$ with $\mu(A)>0$, then

$$
\lim _{N \rightarrow \infty} \frac{1}{N} \sum_{n=1}^{N} \mu\left(A \cap T^{[g(n)]} A\right) \geq(\mu(A))^{2} .
$$

Proof: By using weak convergence in the above theorem we get that for any ergodic system $(X, \mathcal{B}, \mu, T), A \in \mathcal{B}$, and $f=1_{A}, \lim _{N \rightarrow \infty} \frac{1}{N} \sum_{n=1}^{N} \mu\left(A \cap T^{[g(n)]} A\right)=\lim _{N \rightarrow \infty} \frac{1}{N} \sum_{n=1}^{N} \int f T^{-[g(n)]} f d \mu=$ $\left(\int f\right)^{2}=(\mu(A))^{2}$. If $(X, \mathcal{B}, \mu, T)$ is not ergodic the inequality (34) follows by utilizing the ergodic decomposition of $\mu$ (see for example [3, Sec.5]).

Theorem 7.3 Let $g_{1}, g_{2}, \ldots, g_{k} \in \mathcal{F}$ be such that $\lim _{x \rightarrow \infty} \frac{g_{i+1}(x)}{g_{i}(x)}=0$ and the ratio $\frac{g_{i+1}^{\prime}(x)}{g_{i}^{\prime}(x)}$ is eventually monotone, $i=1, \ldots, k-1$. Then for any invertible ergodic system $(X, \mathcal{B}, \mu, T)$ and any $f_{1}, \ldots, f_{k} \in L^{\infty}(X, \mathcal{B}, \mu)$,

$$
\lim _{N \rightarrow \infty}\left\|\frac{1}{N} \sum_{n=1}^{N} T^{\left[g_{1}(n)\right]} f_{1} \cdots T^{\left[g_{k}(n)\right]} f_{k}-\prod_{i=1}^{k} \int f_{i}\right\|_{2}=0
$$


Theorem 7.3 is a corollary of the following more general result.

Lemma 7.4 Let either $g_{1}(x)=x$ or $g_{1} \in \mathcal{F}$, and let $g_{2}, \ldots, g_{k} \in \mathcal{F}, g_{k+1} \in \mathcal{F} \cup\{0\}$, so that $\lim _{x \rightarrow \infty} \frac{g_{i+1}(x)}{g_{i}(x)}=0$, and such that the ratio $\frac{g_{i+1}^{\prime}(x)}{g_{i}^{\prime}(x)}$ is eventually monotone, $i=1, \ldots, k$. Let also for $l \geq 0$ and $i=1, \ldots, l, g_{1 i} \in \mathcal{F}$, so that $\lim _{x \rightarrow \infty} \frac{g_{1 i}(x)}{g_{k}(x)}=0$, and such that the ratios $\frac{g_{1 i}(x)}{g_{k}(x)}$ and $\frac{g_{1 i}^{\prime}(x)}{g_{k}^{\prime}(x)}$ are eventually monotone. Then for any invertible ergodic system $(X, \mathcal{B}, \mu, T)$ and any $f_{1}, \ldots, f_{k} \in L^{\infty}(X, \mathcal{B}, \mu)$,

$$
\lim _{N \rightarrow \infty}\left\|\frac{1}{N} \sum_{n=1}^{N} T^{\left[g_{1}(n)\right]-\sum_{i=1}^{l}\left[g_{1 i}(n)\right]} f_{1} T^{\left[g_{2}(n)\right]-\left[g_{k+1}(n)\right]} f_{2} \cdots T^{\left[g_{k}(n)\right]-\left[g_{k+1}(n)\right]} f_{k}-\prod_{i=1}^{k} \int f_{i}\right\|_{2}=0 .
$$

Proof: In the proof we will use the following facts repeatedly. First, if $g \in \mathcal{F}$ and $h \in \mathbf{N}$, then $[g(n+h)]-[g(n)]=0$ for a set of $n$ of density 1, by Lemma 2.12. Second, if $g_{1}, g_{2} \in \mathcal{F}$, $\lim _{x \rightarrow \infty} \frac{g_{2}(x)}{g_{1}(x)}=0$ and $\frac{g_{2}^{\prime}(x)}{g_{1}^{\prime}(x)}$ is eventually monotone, then $\hat{g}_{2}(x)=g_{2}\left(g_{1}^{-1}(x)\right) \in \mathcal{F}$ and $\left[g_{2}(n)\right]=$ $\left[\hat{g}_{2}\left(\left[g_{1}(n)\right]\right)\right]$ on a set of $n$ of density 1 , by Lemma 2.7 .

The proof is by induction on $k$. To prove the base of induction, $k=1$, we have two cases to check.

Case 1, when $g_{1}(n)=n$. If $x_{n}=T^{n-\sum_{i=1}^{l}\left[g_{1 i}(n)\right]} f, \int f=0$, then $\left\langle x_{n}, x_{n+h}\right\rangle=\int f T^{h} f d \mu$ on a set of $n$ of density 1. So $\lim _{H \rightarrow \infty} \frac{1}{H} \sum_{h=1}^{H} \lim _{N \rightarrow \infty} \frac{1}{N} \sum_{n=1}^{N}\left\langle x_{n}, x_{n+h}\right\rangle=\left(\int f\right)^{2}=0$ by ergodicity of $T$. Hence, by van der Corput's theorem, $\lim _{N \rightarrow \infty}\left\|\frac{1}{N} \sum_{n=1}^{N} x_{n}\right\|_{2}=0$. Note that this result would not be true for all ergodic $T$ if $g_{1}(n)=a n, a>1$.

Case 2, when $g_{1} \in \mathcal{F}$. Let $m=\left[g_{1}(n)\right], \phi(m)=\left|\left\{n \mid m=\left[g_{1}(n)\right]\right\}\right|$ and $\hat{g}_{1 i}(x)=g_{1 i}\left(g_{1}^{-1}(x)\right)$, $i=1, \ldots, l$. Then

$$
\begin{aligned}
\lim _{N \rightarrow \infty}\left\|\frac{1}{N} \sum_{n=1}^{N} T^{\left[g_{1}(n)\right]-\sum_{i=1}^{l}\left[g_{1 i}(n)\right]} f\right\|_{2} & =\lim _{N \rightarrow \infty}\left\|\frac{1}{N} \sum_{m=1}^{\left[g_{1}(N)\right]} \phi(m) T^{m-\sum_{i=1}^{l}\left[\hat{g}_{1 i}(m)\right]} f\right\|_{2} \\
& =\lim _{N \rightarrow \infty}\left\|\frac{1}{N} \sum_{n=1}^{N} T^{n-\sum_{i=1}^{l}\left[\hat{g}_{1 i}(n)\right]} f\right\|_{2}
\end{aligned}
$$

according to the lemmas 2.4, 2.5, 2.7 and Theorem 3.5. We are back to case 1 .

Suppose now that the $k-1$ case is proved. Let

$$
x_{n}=T^{\left[g_{1}(n)\right]-\sum_{i=1}^{l}\left[g_{1 i}(n)\right]} f_{1} T^{\left[g_{2}(n)\right]-\left[g_{k+1}(n)\right]} f_{2} \cdots T^{\left[g_{k}(n)\right]-\left[g_{k+1}(n)\right]} f_{k}
$$

where we may assume that $\int f_{1}=0$. Again, if $g_{1}(n)=n$, then

$$
\begin{aligned}
\lim _{N \rightarrow \infty} & \frac{1}{N} \sum_{n=1}^{N}\left\langle x_{n}, x_{n+h}\right\rangle \\
= & \lim _{N \rightarrow \infty} \frac{1}{N} \sum_{n=1}^{N} \int T^{n-\sum_{i=1}^{l}\left[g_{1 i}(n)\right]}\left(f_{1} T^{h} f_{1}\right) T^{\left[g_{2}(n)\right]-\left[g_{k+1}(n)\right]} f_{2}^{2} \cdots T^{\left[g_{k}(n)\right]-\left[g_{k+1}(n)\right]} f_{k}^{2} d \mu \\
= & \lim _{N \rightarrow \infty} \frac{1}{N} \sum_{n=1}^{N} \int T^{n-\tilde{g}(n)}\left(f_{1} T^{h} f_{1}\right) T^{\left[g_{2}(n)\right]-\left[g_{k}(n)\right]} f_{2}^{2} \cdots T^{\left[g_{k-1}(n)\right]-\left[g_{k}(n)\right]} f_{k-1}^{2} \cdot f_{k}^{2} d \mu
\end{aligned}
$$




$$
=\int f_{1} T^{h} f_{1} d \mu \int f_{2}^{2} \cdots \int f_{k}^{2}
$$

by the induction hypothesis, where $\tilde{g}(n)=\sum_{i=1}^{l}\left[g_{1 i}(n)\right]-\left[g_{k}(n)\right]+\left[g_{k+1}(n)\right]$. Hence, by ergodicity of $T$,

$$
\lim _{H \rightarrow \infty} \frac{1}{H} \sum_{h=1}^{H} \lim _{N \rightarrow \infty} \frac{1}{N} \sum_{n=1}^{N}\left\langle x_{n}, x_{n+h}\right\rangle=\left(\int f_{1}\right)^{2} \int f_{2}^{2} \cdots \int f_{k}^{2}=0 .
$$

If $g_{1} \in \mathcal{F}$ then let, as in the case $k=1, m=\left[g_{1}(n)\right]$ so that

$$
\begin{aligned}
\lim _{N \rightarrow \infty}\left\|\frac{1}{N} \sum_{n=1}^{N} x_{n}\right\|_{2} & =\lim _{N \rightarrow \infty}\left\|\frac{1}{N} \sum_{m=1}^{\left[g_{1}(N)\right]} \phi(m) T^{m-\sum_{i=1}^{l}\left[\hat{g}_{1 i}(m)\right]} f_{1} \cdots T^{\left[\hat{g}_{k}(m)\right]-\left[\hat{g}_{k+1}(m)\right]} f_{k}\right\|_{2} \\
& =\lim _{N \rightarrow \infty}\left\|\frac{1}{N} \sum_{n=1}^{N} T^{n-\sum_{i=1}^{l}\left[\hat{g}_{1 i}(n)\right]} f_{1} \cdots T^{\left[\hat{g}_{k}(n)\right]-\left[\hat{g}_{k+1}(n)\right]} f_{k}\right\|_{2}
\end{aligned}
$$

by Theorem 3.5. We are now back to the previous situation.

For example, Theorem 7.3 is true for

- $g_{i}(x)=x^{\alpha_{i}} \phi_{i}(x), i=1, \ldots, k$, where $1>\alpha_{1}>\alpha_{2}>\cdots>\alpha_{k}>0$ and $\phi_{1}, \ldots, \phi_{k} \in \mathcal{S}$.

- $g_{i}(x)=x^{\alpha} \log ^{\beta_{i}} x, i=1, \ldots, k$, where $0<\alpha<1$ and $\beta_{1}>\beta_{2}>\cdots>\beta_{k}$.

In a similar way as we got Corollary 7.2 we now have

Corollary 7.5 (Cf. [5, Corollary, p.32]) Let $g_{1}, g_{2}, \ldots, g_{k} \in \mathcal{F}$ satisfy the assumptions of Theorem 7.3. Then for any invertible measure preserving system $(X, \mathcal{B}, \mu, T)$ and any $A \in \mathcal{B}$, $\mu(A)>0$,

$$
\lim _{N \rightarrow \infty} \frac{1}{N} \sum_{n=1}^{N} \mu\left(A \cap T^{\left[g_{1}(n)\right]} A \cap \cdots \cap T^{\left[g_{k}(n)\right]} A\right) \geq(\mu(A))^{k+1} .
$$

The equality in formula (35) holds for all $A \in \mathcal{B}$ if and only if $T$ is ergodic.

Corollary 7.5, being a result about an arbitrary probability measure-preserving system has an interesting application to combinatorial number theory.

By Furstenberg's correspondence principle (see [7, Prop. 7.2], [4, p. 755-756]), given a set $E \subset \mathbf{N}$ with $\bar{d}(E)>0$ (see definition of $\bar{d}(E)$ in footnote 5) one can find an invertible probability measure preserving system $(X, \mathcal{B}, \mu, T)$ and a set $A \in \mathcal{B}$ with $\mu(A)=\bar{d}(E)$, such that for any $k \in \mathbf{N}$ and any $n_{1}, n_{2}, \ldots, n_{k} \in \mathbf{Z}$, one has

$$
\mu\left(A \cap T^{-n_{1}} A \cap \cdots \cap T^{-n_{k}} A\right) \leq \bar{d}\left(E \cap\left(E-n_{1}\right) \cap \cdots \cap\left(E-n_{k}\right)\right) .
$$

We see now that Corollary 7.5 implies the following result. 
Corollary 7.6 Let $E \subset \mathbf{N}$ be a subset with $\bar{d}(E)>0$. If $g_{1}, g_{2}, \ldots, g_{k} \in \mathcal{F}$ satisfy the assumptions of Theorem 7.3 then one has

$$
\underline{d}\left(\left\{n \in \mathbf{N} \mid \bar{d}\left(E \cap\left(E-\left[g_{1}(n)\right]\right) \cap \cdots \cap\left(E-\left[g_{k}(n)\right]\right)\right) \geq(\bar{d}(E))^{k+1}\right\}\right)>0 .
$$

Remark: For a Szemerédi type result involving multiples of a tempered Hardy function see the recent preprint [12].

\section{Some conjectures}

In this short final section we formulate two natural conjectures.

Conjecture 8.1 Let each of $g_{1}, \ldots, g_{k}$ and $g_{i}-g_{j}, i \neq j, 1 \leq i, j \leq k$, be either a tempered function or a non-constant polynomial. Then for any invertible weakly mixing system $(X, \mathcal{B}, \mu, T), i=1, \ldots, k$, one has

$$
\lim _{N \rightarrow \infty}\left\|\frac{1}{N} \sum_{n=1}^{N} T^{\left[g_{1}(n)\right]} f_{1} T^{\left[g_{2}(n)\right]} f_{2} \cdots T^{\left[g_{k}(n)\right]} f_{k}-\prod_{i=1}^{k} \int f_{i} d \mu\right\|_{2}=0 .
$$

The following conjecture is motivated by Corollary 7.5.

Conjecture 8.2 Let $g_{1}, g_{2}, \ldots, g_{k} \in \mathcal{T} \cap \mathcal{H}$ be such that $\lim _{x \rightarrow \infty} \frac{g_{i+1}(x)}{g_{i}(x)}=0, i=1, \ldots, k-1$. Then for any invertible measure preserving system $(X, \mathcal{B}, \mu, T)$ and any $A \in \mathcal{B}, \mu(A)>0$,

$$
\lim _{N \rightarrow \infty} \frac{1}{N} \sum_{n=1}^{N} \mu\left(A \cap T^{\left[g_{1}(n)\right]} A \cap \cdots \cap T^{\left[g_{k}(n)\right]} A\right) \geq(\mu(A))^{k+1} .
$$

\section{References}

[1] D. Berend and V. Bergelson, Mixing sequences in Hilbert spaces, Proc. Amer. Math. Soc. 98 (1986), 239-246.

[2] V. Bergelson, Weakly mixing PET, Ergod. Th. \& Dynam. Sys. 7 (1987), 337-349.

[3] V. Bergelson, The multifarious Poincaré recurrence theorem, Descriptive set theory and dynamical systems (ed. M. Foreman, A. Kechris, A. Louveau, B. Weiss), London Math. Soc. Lecture Note Series 277 (2000), 31-57.

[4] V. Bergelson, Combinatorial and Diophantine applications of ergodic theory. Appendix A by A. Leibman and Appendix B by Anthony Quas and Máté Wierdl. Handbook of dynamical systems. Vol. 1B, (ed. B. Hasselblatt and A. Katok), Elsevier B. V., Amsterdam, 2006, $745-869$.

[5] V. Bergelson, M. Boshernitzan and J. Bourgain, Some results on nonlinear recurrence, J. Anal. Math. 62 (1994), 29-46. 
[6] V. Bergelson and A. Leibman, Polynomial extensions of van der Waerden's and Szemerédi's theorems, J. Amer. Math. Soc. 9 (1996), 725-753.

[7] V. Bergelson and R. McCutcheon, An ergodic IP polynomial Szemerédi theorem, Mem.Amer.Math.Soc. 146 (695) (2000), viii+106 pp.

[8] J. Boos, Classical and Modern Methods in Summability, Oxford University Press, 2000.

[9] M. Boshernitzan, G. Kolesnik, A. Quas and M. Wierdl, Ergodic averaging sequences, J. D'Analyse Mathematique, vol. 95 (2005), 63-103.

[10] N. Bourbaki, Fonctions d'une variable réele. Chapitre V (Étude Locale des Fonctions), 2nd edition, Herman, Paris, 1961.

[11] J. Cigler, Some remarks on the distribution mod 1 of tempered sequences, Nieuw Archief voor Wiskunde (3), 16 (1968), 194-196.

[12] N. Frantzikinakis and M. Wierdl, A Hardy field extension of Szemerédi's theorem. Preprint. Available at http://arxiv.org/abs/0802.2734.

[13] H. Furstenberg, Ergodic behavior of diagonal measures and a theorem of Szemerédi on arithmetic progressions, J. d'Analyse Math. 31 (1977), 204-256.

[14] H. Furstenberg, Recurrence in Ergodic Theory and Combinatorial Number Theory, Princeton Univ. Press, Princeton, N.J., 1981.

[15] G. H. Hardy, Orders of infinity, Cambridge Tracts in Math. and Math.Phys.12, 2nd edition, Cambridge, 1924.

[16] G. H. Hardy, Divergent Series, Oxford Univ. Press, London, 1949

[17] E. Hlawka, The Theory of Uniform Distribution, AB Academic Publishers, 1984

[18] L.K. Jones and M. Lin, Ergodic theorems for weak mixing type, Proc. AMS, 57 (1976), $50-52$.

[19] L. Kuipers and H. Niederreiter, Uniform Distribution of Sequences, J. Wiley \& Sons, 1974

[20] G. G. Lorentz, A contribution to the theory of divergent sequences, Acta Math. 80 (1948), 167-190.

[21] J. G. van der Corput, Diophantische Ungleichungen I. Zur Gleichverteilung modulo Eins, Acta Math. 56 (1931), 373-456. 